University of Louisville

ThinkIR: The University of Louisville's Institutional Repository

Electronic Theses and Dissertations

$12-2019$

\title{
Therapeutic potential of withaferin A against non-small cell lung cancer.
}

Al Hassan Kyakulaga

University of Louisville

Follow this and additional works at: https://ir.library.louisville.edu/etd

Part of the Pharmacology, Toxicology and Environmental Health Commons

\section{Recommended Citation}

Kyakulaga, Al Hassan, "Therapeutic potential of withaferin A against non-small cell lung cancer." (2019). Electronic Theses and Dissertations. Paper 3311.

https://doi.org/10.18297/etd/3311

This Doctoral Dissertation is brought to you for free and open access by ThinkIR: The University of Louisville's Institutional Repository. It has been accepted for inclusion in Electronic Theses and Dissertations by an authorized administrator of ThinkIR: The University of Louisville's Institutional Repository. This title appears here courtesy of the author, who has retained all other copyrights. For more information, please contact thinkir@louisville.edu. 
THERAPEUTIC POTENTIAL OF WITHAFERIN A AGAINST NON-SMALL CELL

\title{
LUNG CANCER
}

\author{
By \\ Al Hassan Kyakulaga \\ MSc. Pharmacology, Makerere University, 2013 \\ MS Pharmacology \& Toxicology, University of Louisville, 2017
}

A Dissertation Submitted to the Faculty of the

School of Medicine of the University of Louisville

In Partial Fulfillment of the Requirements

for the Degree of

Doctor of Philosophy in Pharmacology and Toxicology

Department of Pharmacology and Toxicology

School of Medicine, University of Louisville

Louisville, KY, USA

December, 2019 

THERAPEUTIC POTENTIAL OF WITHAFERIN A AGAINST NON-SMALL CELL LUNG CANCER

\author{
By \\ Al Hassan Kyakulaga \\ A Dissertation Approved on \\ August 29, 2019 \\ By the following Dissertation Committee:
}

Ramesh C. Gupta, Ph.D.

Jason Chesney, M.D., Ph.D.

Jun Yan, M.D., Ph.D.

Frederick A. Luzzio., Ph.D

Farrukh Aqil, Ph.D. 


\section{DEDICATION}

This doctoral thesis is dedicated to Elianah, Elynn, and Nancy for their love, support and perseverance. 


\section{ACKNOWLEDGEMENTS}

I would like to extend my sincere gratitude to my mentor, Dr. Ramesh C. Gupta for his enthusiasm, guidance, support, and interest in my academic and professional training at the University of Louisville. I am truly indebted for the amazing opportunity he has provided with me the past five years to work in his laboratory. My acknowledgments also go to Drs. Farrukh Aqil and Radha Munagala for their tireless efforts towards teaching me skills that have enabled me to accomplish this work. Also, I would like to thank my lab former mates - Jeyaprakash Jeyabalan, Ashley Mudd, Divya Karukonda and Dr. Ashish Agrawal for the continuous support they have rendered to me during the conduct of these experiments.

I am very grateful with the financial support that I received the Department of Pharmacology and Toxicology, Integrated Programs in Biomedical Sciences (IPBS) program, the Agnes Brown Duggan Endowment, and the School of Graduate Studies at the University of Louisville for the Graduate Fellowship. My special thanks go to Dr. David Hein (Chair, Pharmacology \& Toxicology), Dr. Christopher States, and Dr. Brian Ceresa for their encouragement, advice, and professional support towards my $\mathrm{Ph} . \mathrm{D}$. training. 


\title{
ABSTRACT \\ THERAPEUTIC POTENTIAL OF WITHAFERIN-A AGAINST NON-SMALL CELL LUNG CANCER:
}

\author{
Al Hassan Kyakulaga
}

August $29^{\text {th }}, 2019$

Lung cancer remains the leading cause of cancer-related deaths among both men and women worldwide, and in the United States. Non-small cell lung cancer (NSCLC) represents about $85-90 \%$ of all lung cancer cases and has an overall five-year survival rate of only $17 \%$. Since $60-70 \%$ of NSCLC patients are diagnosed when the tumors are at an advanced stage, chemotherapy remains the most viable treatment option for NSCLC. Recently, the development of molecularly targeted drugs and the breakthrough immunotherapies have shifted the front-line therapies for NSCLC from cytotoxic chemotherapy towards an era of personalized medicine. However, due to the high genetic and cellular heterogeneity of NSCLC tumors, these revolutionary therapies are not applicable in $>70 \%$ of NSCLC patients. Moreover, regardless of treatment approach, dose-limiting toxicity and drug resistance are major therapeutic challenges in treatment of advanced NSCLC. Therefore, there is a significant unmet clinical need to develop highly efficacious therapeutic options for the treatment of advanced NSCLC. In this dissertation, studies were performed with the overall objective of determining the therapeutic potential of withaferin A (WFA), a plant-derived steroidal lactone anticancer compound against NSCLC. 
Briefly, the preclinical efficacy, toxicity and pharmacokinetic properties of WFA were investigated using in vitro and in vivo models. Two human NSCLC cell lines, H1299, and A549 were used to investigate the antitumor efficacy of WFA, alone or in combination with paclitaxel (PAC), a standard chemotherapeutic agent used for the treatment of various types of cancer. Firstly, we found that WFA alone significantly inhibited $\left(\mathrm{IC}_{50}: 0.3-0.6 \mu \mathrm{M}\right)$ the growth, proliferation, colony formation, adhesion, migration, and invasion of both H1299 and A549 cells. As reported elsewhere, the antitumor activities of WFA were both time- and concentration-dependent, but were completely abrogated in the presence of thiol-containing compounds such as $\mathrm{N}$-acetyl cysteine (NAC) and dithiothreitol (DTT). Importantly, the combination of PAC and WFA at 1:40, 1:20, and 1:10 ratios, respectively, resulted in synergistic inhibition of tumor cell growth and proliferation for both H1299 and A549 cell lines. Comparatively, the PAC+WFA combination displayed greater anticancer efficacy and potency than cisplatin (cis-Pt) alongside $\mathrm{PAC}$ (cis-Pt+PAC) or cis-Pt+WFA, and it resulted in a >40-fold decrease in the $\mathrm{IC}_{50}$ of PAC against both $\mathrm{H} 1299$ and A549 cells. In addition, using PAC or taxol resistant cell lines (TR-A549), we demonstrated that WFA was active against the growth and proliferation of chemotherapy resistant human NSCLC cells both in vitro and in vivo. Mechanistically, WFA induced apoptosis, modulated the expression of MDR-1, as well as inhibited the induction of epithelial-to-mesenchymal transition (EMT) in A549 NSCLC cells via the regulation of $\operatorname{Smad} 2 / 3$ and NFKB signaling. In the toxicity evaluation, we found that oral administration of up to $12 \mathrm{mg} / \mathrm{kg}$ of WFA was well tolerated with no signs of systemic and histopathologic toxicity in male SD rats. Also, a highly sensitive, reproducible and robust HPLC-MS/MS method for the detection and quantification of WFA in plasma and tissues was developed. Pharmacokinetically, WFA displayed a high plasma protein binding capacity (>95\%) and high in vitro intrinsic clearance (Clint: $88 \mu \mathrm{L} / \mathrm{min} / \mathrm{mg}$ ) in SD rat liver microsomes. Following a single intravenous bolus dose $(10 \mathrm{mg} / \mathrm{kg})$, plasma 
concentration-time profile of WFA showed a very rapid clearance from plasma ( $\mathrm{t} 1 / 2=37$

min). Together, the findings presented in this dissertation demonstrate the therapeutic potential of WFA, and provide a strong rationale for the further studies to develop WFA alone or its combination with standard chemotherapeutic agents such as PAC for the treatment of advanced NSCLC and other cancer types. 
TABLE OF CONTENTS

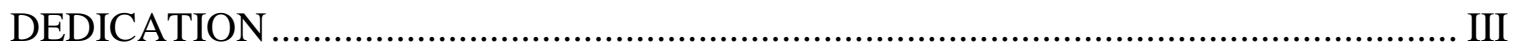

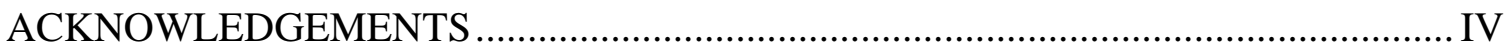

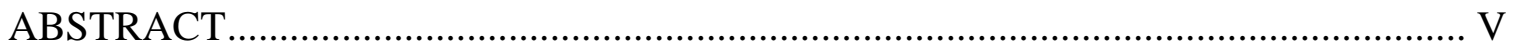

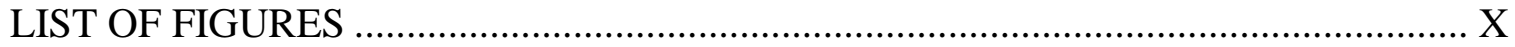

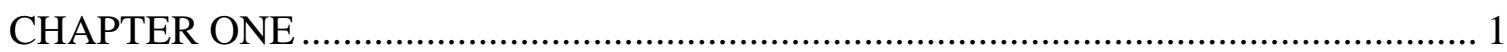

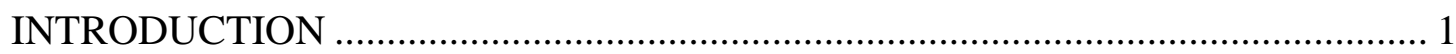

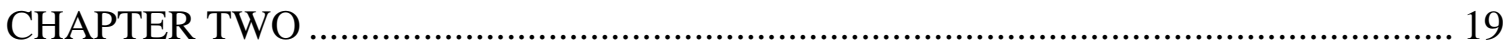

SYNERGISTIC COMBINATIONS OF PACLITAXEL AND WITHAFERIN A AGAINST HUMAN NON-SMALL CELL LUNG CANCER CELLS........................... 19

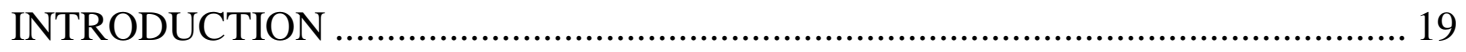

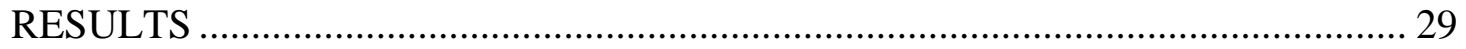

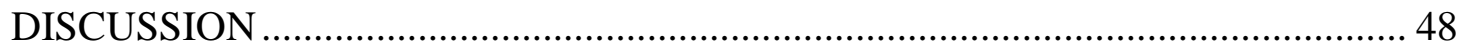

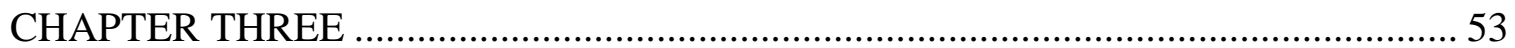

WITHAFERIN A INHIBITS EPITHELIAL TO MESENCHYMAL TRANSITION IN NON-SMALL CELL LUNG CANCER CELLS …………....................................... 53

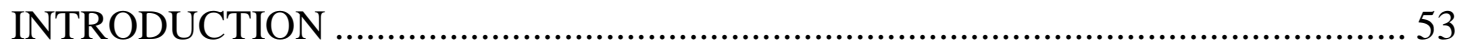




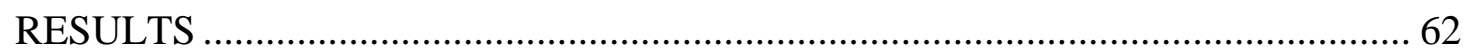

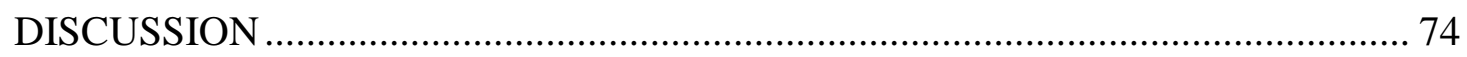

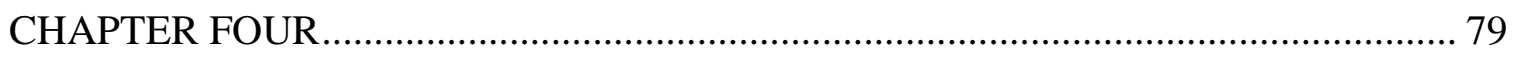

REPEATED DOSING TOXICITY AND PHARMACOKINETICS OF WITHAFERIN A

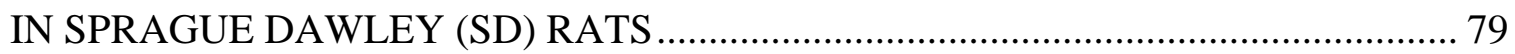

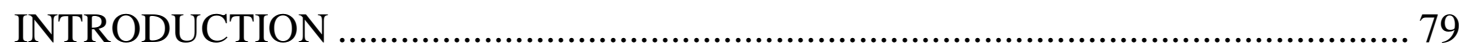

MATERIALS AND METHODS ................................................................ 82

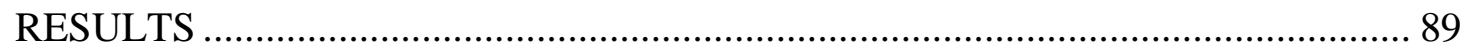

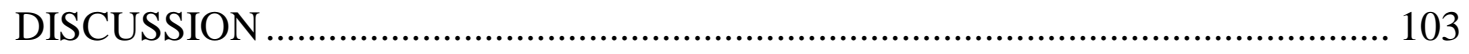

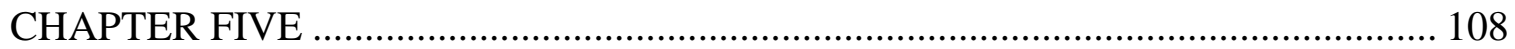

OVERALL DISCUSSION, SUMMARY, AND CONCLUSIONS ........................... 108

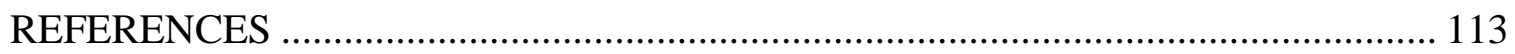

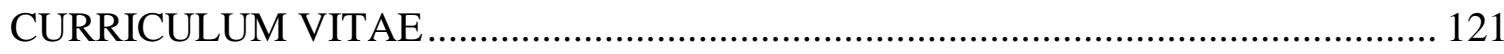




\section{LIST OF FIGURES}

Fig 1.1. Histologic classification of Lung Cancer...................................4

Fig 2.1. Effect of WFA proliferation of $\mathrm{H} 1299$ and A549 cells....................... 31

Fig 2.2. Synergistic effect of WFA, PAC and cis-Pt on H1299 cells......................

Fig 2.3. Synergistic effect of WFA, PAC and cisPt on A549 cells.......................

Fig 2.4. PAC and WFA synergistically inhibited colony formation $\ldots \ldots \ldots \ldots \ldots \ldots \ldots . .40$

Fig 2.5. Effect of cell number on the synergism of PAC and WFA ..................41

Fig 2.6. PAC and WFA inhibited motility and migration...........................43

Fig 2.7. MDR1-dependent PAC chemoresistance in NSCLC cells ....................46

Fig 2.8. WFA inhibited the proliferation of resistant PAC resistant A549 cells..............47

Fig 3.1. WFA suppressed the growth and proliferation of A549 and H1299 cells........63

Fig 3.2. WFA inhibited cell adhesion, motility, migration, and invasion...............65

Fig 3.3. WFA inhibited EMT induction in A549 and H1299 cells.......................67

Fig 3.4. WFA suppressed TGF $\beta 1 / \mathrm{TNF} \alpha$-induced motility ..........................69

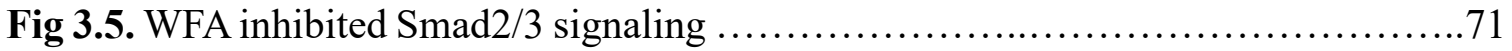

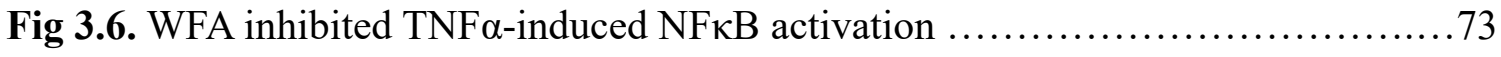

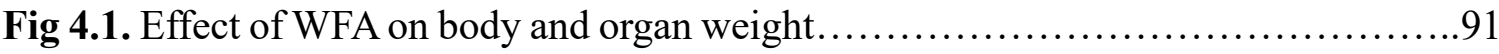

Fig 4.2. Effect of WFA on hematological parameters........................... 92

Fig 4.3. WFA did not cause biochemical or histological toxicity $\ldots \ldots \ldots \ldots \ldots \ldots \ldots \ldots 93$

Fig 4.4. HPLC-MS/MS analysis of WFA ....................................... 95 
Fig 4.5. Tissue and plasma processing scheme.

Fig 4.6. Sample matrix cleanup using Captiva-EMR Lipid SPE........................98

Fig 4.7. In vitro stability of WFA in liver microsomes and plasma protein binding.......100

Fig 4.8. Plasma pharmacokinetics of WFA in male SD rats........................ 102 


\section{LIST OF TABLES}

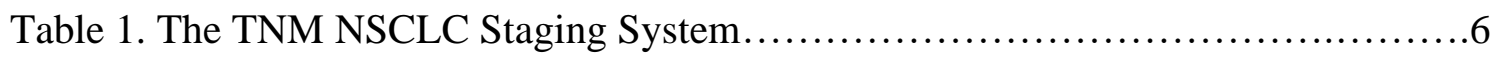

Table 2.1. Synergism Summary Table H1299 Cells...................................39

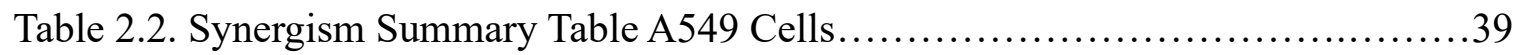




\section{CHAPTER ONE}

\section{INTRODUCTION}

\section{Lung Cancer}

Lung cancer is the leading cause of cancer-related deaths among both men and women worldwide [1, 2], and in the United States [3]. In 2018, there were about 2.1 million new cases (about $11.6 \%$ of all cancer cases) and 1.76 million deaths due to lung cancer reported worldwide [4]. In the United States, approximately 234,030 new cases $(121,680$ men and 112,350 women) and 154,050 deaths (83,550 men and 70,500 women) due to lung cancer were estimated to have occured in 2018 [3]. On the basis of these statistics, more people have died from lung cancer than from breast, pancreatic, prostate and colon cancers combined $[3,5]$. This extremely poor prognosis from lung cancer is driven in part by the complexity and biological aggressiveness of lung tumors [5-7]. In addition, the majority of lung cases are either locally advanced or highly metastatic at the time of diagnosis [8] and are never curable regardless of treatment approach $[9,10]$. Thus, although there have been notable improvements in the progression-free survival rates, the overall survival from lung cancer has remained dismal [3]. Therefore, unless new treatments are developed, there remains a significant unmet clinical need for the development of clinically effective therapeutic strategies to change the current clinical landscape of lung cancer. 
Clinically, lung cancer is a highly heterogeneous and complex disease, representing a large group of tumor types that develop from cells in the lung tissue $[5,7,11]$. Although there are many subtypes, lung tumors almost exclusively (>99\%) develop from airway epithelial cells [7] and are generally termed as carcinomas. The high tumor heterogeneity, coupled with the biological aggressiveness of lung cancer make the treatment choices very difficult, subjective and ineffective $[9,11,12]$. As such, to effectively treat these multiple lung cancer subtypes is a major challenge which demands a spectrum of treatment options and approaches. Additionally, lung tumors are largely asymptomatic in the early stages [6, 11] and as a result are mostly diagnosed when the disease is at an advanced stage. Thus, despite the advancements in imaging techniques and molecular diagnostics, current clinical reports indicate that $60-70 \%$ of lung cancer cases are diagnosed at stage III-IV [3, 13]. Since curative surgery is not applicable in patients with advanced lung cancer, almost all stage III-IV lung cancer patients succumb to the disease within a year of diagnosis $[9,13]$. Unfortunately, even among patients that present with early-stage disease, there are very high tumor relapse rates which contribute to high mortality rates [14].

Based on the cellular histology (Fig. 1.1), the World Health Organization (WHO) classifies lung cancer as either small-cell lung cancer (SCLC) or non-small cell lung cancer (NSCLC) [5, 10]. In the US and worldwide, NSCLC tumors account for 80-90\% of all lung cancer cases [3]. The SCLC tumors primarily occur among heavy smokers and appear as small round-shaped tumor cells, with visible mitotic patterns, granular nuclear chromatin and absent nucleoli $[6,7]$. On the other hand, NSCLC tumor cells are much more complex [5] and are further sub-divided into three main subtypes; adenocarcinomas, squamous-cell carcinomas and large-cell carcinomas [15]. Currently [5], adenocarcinomas are the most 
prevalent subtype of NSCLC $[2,10]$ and these present as glandular or mucin-producing cells. Histologically, the appearance of tumor cells in adenocarcinomas is such that the cells are arranged as; (a) acinar, (b) papillary, (c) bronchoalveolar, or a mixture of these growth patterns (Fig. 1.1) [10]. Contrastingly, the appearance of squamous cell carcinomas, the second most prevalent subtype of NSCLC, is such that the cells display intercellular bridges and/or keratin. Finally, large-cell carcinomas, the third subtype of NSCLC are visualized as large tumor cells containing vesicular nuclei and prominent nucleoli. Overall, the high degree of cellular and genetic complexity of lung tumors make treatment options extremely challenging and accounts for the poor prognostic outcomes [16, 17].

Etiologically, the carcinogenesis of lung cancer is a complex and multifactorial process that is driven by an interplay of several environmental, genetic and lifestyle factors $[6,10]$. To date, cigarette smoking remains by far the major cause for lung cancer, and worldwide statistics $[1,2,10]$ show a significant positive correlation between smoking patterns and lung cancer incidence rates. These data indicate that both active and sidestream (or the so called "passive") smoking cause lung cancer [6, 7]. Quantitatively, the risk for lung cancer increases with both the quantity and duration of smoking, and as such is highest among those who smoke and never quit. In the United States, it has been estimated that almost $90 \%$ of all lung cancer cases are attributable to cigarette smoking [18] [3]. Chemically, cigarette smoke contains a complex mixture of many known carcinogens (such as benzo $[a]$ pyrene and, nitrosamines) $[6,7]$. In susceptible individuals, exposure to these carcinogens cause specific genetic mutations and/or epigenetic changes in cells that result in alteration of cellular-signaling mechanisms. Studies have shown that early in the carcinogenic process [6], these changes in cell signaling mechanisms confer a 
proliferative advantage to a small group of lung epithelial cells that are later progressively transformed into highly malignant lung tumor cells $[7,11]$. In addition to carcinogens in cigarette smoke, other risk factors for lung cancer include exposure to asbestos, chromium, arsenic, nickel, cadmium, beryllium, silica, and radon gas [10].

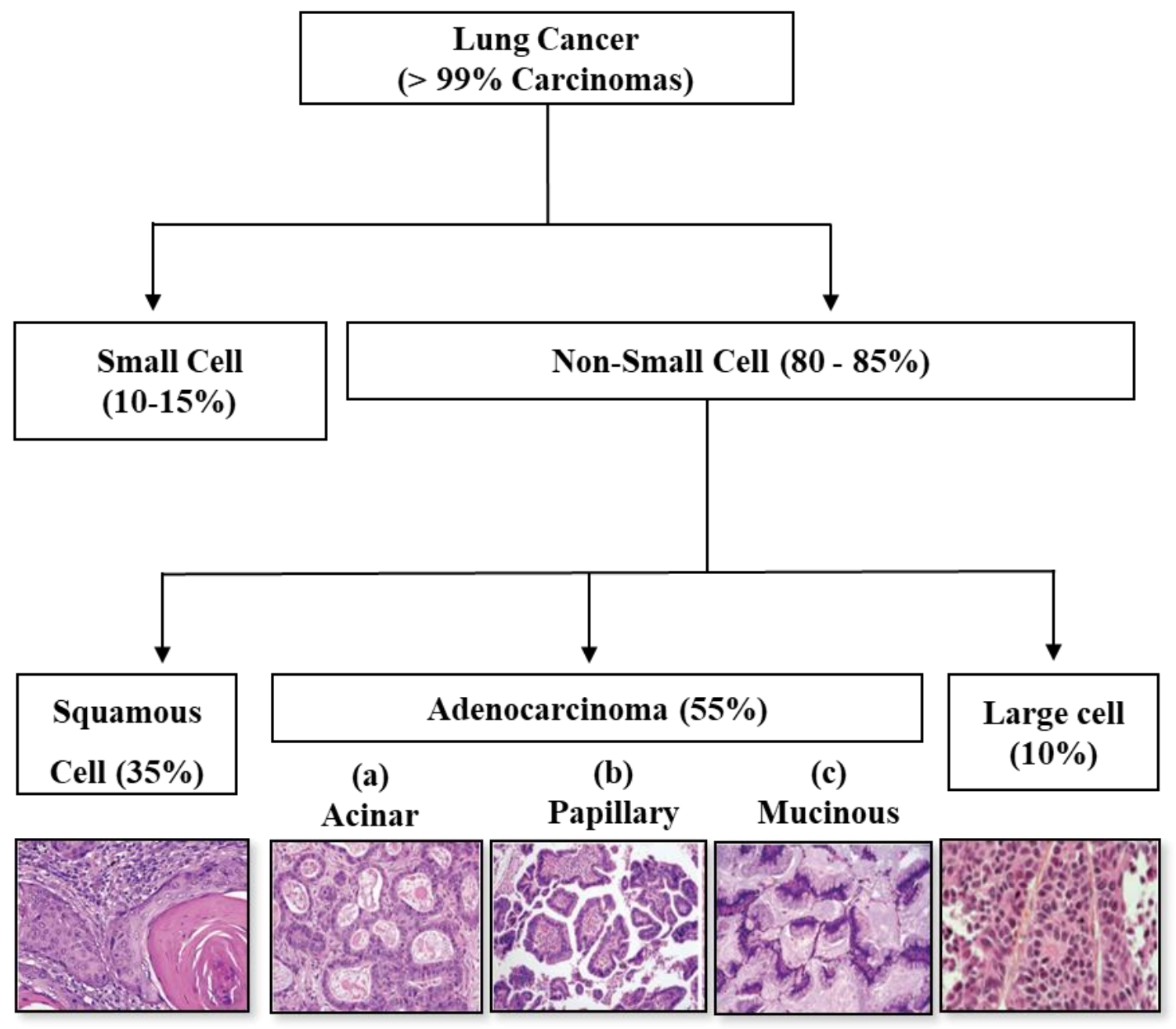

Fig 1.1. Histologic classification of Lung Cancer. 


\section{The NSCLC Staging system}

The staging of cases at diagnosis is very critical in the treatment of NSCLC.

Currently, the choice of therapeutic strategy and the expected clinical prognosis for NSCLC are mainly dependent on the stage of the disease at diagnosis. The most widely utilized and recommended staging criteria for NSCLC tumors at first diagnosis is the Tumor-Node-Metastasis (TNM) system (Table 1). In this staging system, the NSCLC tumors are assigned a series of descriptors or scores which enable oncologists to group the NSCLC tumors into seven clinical stages that reflect the expected clinical outcomes [11]. Numerical clinical scores are assigned to the size of the primary tumor (T), the presence of tumor metastases in the regional lymph nodes $(\mathrm{N})$ and in distant organs $(\mathrm{M})[19,20]$. Together, the combination of these scores allows the NSCLC cases to be grouped into the seven stages; IA, IB, IIA, IIB, IIIA, IIIB, and V. For prognostic purposes, the NSCLC cases assigned to stages IA, IB, IIA, and IIB are generally considered to be in the early-stages of the disease. For these early-stages of NSCLC, the primary tumors are associated with no evidence of local or distant metastases $[20,21]$. On the other hand, NSCLC cases that display tumor descriptors which fall into the stages III-V display evidence of metastatic nodules in the regional lymph nodes and/or distant organs. Therefore, the stages III-V of NSCLC are considered to be advanced stages of the disease Un and these usually have the worst survival outcomes [20]. Unfortunately, in the clinic, the majority of NSCLC cases $(60-70 \%)$ are diagnosed when the tumors are at stage IV-V and are considered to be advanced NSCLC. 
Table 1. The TNM NSCLC Staging System

\begin{tabular}{|c|c|}
\hline Stage & TNM \\
\hline Stage 0 & Carcinoma in situ \\
\hline Stage 1A & T1 N0 M0 \\
\hline Stage 1B & T2 N0 M0 \\
\hline \multirow[t]{2}{*}{ Stage IIA } & T1 N1 M0 \\
\hline & T2 N1 M0 \\
\hline \multirow[t]{3}{*}{ Stage IIB } & T3 N0 M0 \\
\hline & T3 N1 M0 \\
\hline & T1 N2 M0 \\
\hline \multirow[t]{5}{*}{ Stage IIIA } & $\mathrm{T} 2 \mathrm{~N} 2 \mathrm{M} 0$ \\
\hline & T3 N2 M0 \\
\hline & T4 N0 M0 \\
\hline & T4 N1 M0 \\
\hline & $\mathrm{T} 4 \mathrm{~N} 2 \mathrm{M} 0$ \\
\hline \multirow[t]{4}{*}{ Stage IIIB } & T1 N3 M0 \\
\hline & $\mathrm{T} 2 \mathrm{~N} 3 \mathrm{M} 0$ \\
\hline & T3 N3 M0 \\
\hline & T4 N3 M0 \\
\hline Stage V & Any T, Any N, M1 \\
\hline
\end{tabular}




\section{The Treatment of NSCLC}

Once the stage of NSCLC is established, the next step is the determination of the most appropriate course of treatment for each patient on a case by case basis. In the clinic, the treatments for NSCLC comprise of surgical removal of part or whole of the lung tissue, radiation, and systemic chemotherapy either alone or in combination $[6,13]$. Per the TNM descriptors, the therapeutic goal for early-stage NSCLC is to cure the patients of the primary tumors. Therefore, for patients that are diagnosed with early stage NSCLC, curative surgery is the standard treatment of choice and this patient population represents those in whom the best clinical outcomes are expected. Indeed, multiple clinical reports have shown that cure of the primary tumors is reportedly attainable in up to $80 \%$ and $50 \%$ for stage I and stage II cases, respectively [13]. Unfortunately, only about 20-25\% of all NSCLC cases are at an early stage (I-II) at diagnosis [2]. Moreover, many of these patients are above 60 years of age and comorbidities such as pulmonary and cardiac disease are not uncommon. As such, in greater than $50 \%$ of early-stage NSCLC patients, surgery is not practical because of the poor overall health condition of the patients or what is clinically referred to as performance status [6]. Furthermore, it is also widely reported that relapse rates in NSCLC after surgery are in excesses of 70\% within 6-12 month. Therefore, surgery by itself is never a sufficient and effective therapeutic strategy for the treatment of NSCLC and most patients require supportive systemic therapies $[13,18,22]$.

Systemic chemotherapy remains the cornerstone in the management of NSCLC in the neoadjuvant, adjuvant and/or palliative settings. Regardless of the stage of NSCLC at diagnosis, almost all NSCLC patients benefit from some form of systemic therapy during the course of the treatment. However, since surgery is not option for patients with advanced 
NSCLC chemotherapy remains the treatment of choice. The main clinical objectives of systemic chemotherapy in advanced NSCLC are to; (1) manage the symptoms, (2) prolong patient survival, and (3) maintain or improve the patient's quality of life. Today, the systemic therapeutic options for NSCLC fall into three broad categories: (1) platinumbased combinations, (2) molecularly targeted drugs, and (3) immune checkpoint inhibitors. Before the selection of the systemic chemotherapeutic regimen in NSCLC, patients are evaluated for several important factors such as tumor histology, patient performance status, the presence of metastasis, and the status driver mutations. Given the biological complexity of NSCLC tumors, no single systemic treatment has been proven clinically effective and as such, the choice of the course of treatment has remained subjective and mainly dependent on the toxicity profile of the drugs used. In the current WHO guidelines, systemic therapy for NSCLC recommends a combination of multiple drugs with different mechanisms of action and therapeutic targets to maximize efficacy, and minimize the risk of overlapping toxicities.

Previously, the choice of systemic chemotherapy for NSCLC was solely dependent on the stage and patient performance status without regard to the differences in the histological subtypes. However, two very significant clinical findings transformed the treatment choices for the various histological subtypes of NSCLC. Firstly, it was observed that the anti-angiogenic agent bevacizumab was differentially toxic among NSCLC with different tumor subtypes. Secondly, in multiple clinical trials it was determined that pemetrexed, a folate anti-metabolite antitumor agent was ineffective in patients with squamous cell carcinoma (SCC), a subtype of NSCLC. Although detailed molecular mechanisms have not been elucidated for these observations, together, these two novel 
clinical findings permanently changed the treatment approaches for the different subtypes of NSCLC. Consequently, while adenocarcinoma, squamous cell carcinoma, and large cell carcinomas are all NSCLC, they each have slightly variable treatment approaches.

In the past decade, the landmark discoveries that revolutionalized the treatment approaches for NSCLC were the molecular identification of activating mutations in genes such as the EGFR and ALK in adenocarcinomas. The discovery of driver mutations in the EGFR and ALK genes have unearthed and fueled the development of new therapeutic paradigms for NSCLC in the form of molecularly targeted drugs. Subsequently, we have witnessed an evolution of molecularly targeted therapies such as tyrosine kinase inhibitors (TKIs) and their accelerated FDA approval as first-line therapies for advanced NSCLC. The introduction of TKIs into the clinic have tremendously shifted the therapeutic options of NSCLC towards an era of personalized medicine. However, these drugs have also been largely ineffective because only $20-30 \%$ of NSCLC cases harbor 'targetable' driver mutations. In addition, besides the high cost of therapy, targeted drugs only offer marginal and temporary improvements in clinical response mainly due to the rapid development of drug resistance and the widely observed disease progression while on treatment.

Specifically, small molecules targeting mutations in the EGFR gene have become very popular as frontline treatments for advanced NSCLC adenocarcinomas. In the US, EGFR mutations represent approximately $10-15 \%$ of adenocarcinoma patients, the most prevalent of which is exon 19 deletions and mutations in exon 21. Extensive molecular studies have showed that these EGFR pathway-activating mutations promote cellular proliferation and the expression of antiapoptotic mechanisms, which together promote tumor progression $[14,25]$. For such patients, agents targeting EGFR mutations have 
become the first-line therapies for NSCLC adenocarcinoma. In this class of anticancer agents, Gefitinib was the first TKI targeting EGFR mutations to receive accelerated FDA approval in 2003 as monotherapy for locally advanced or metastatic NSCLC. Gefitinib was found to be highly effective in NSCLC patients with tumors haboring EGFR mutations after the failure of both platinum-based and docetaxel therapies. In 2004, another EGFR inhibitor, Erlotinib was the first oral anti-EGFR TKI agent to be approved by the FDA for the treatment of advanced NSCLC after treatment failure by platinum-based therapies. Later, the agents such as afatinib, osmertinib, and rociletinib all of which target EGFR have been approved, and alongside gefitinib and erlotinib are recommended as the first-line chemotherapeutic agents for the treatment of advanced NSCLC adenocarcinoma with EGFR activation mutations. Unfortunately, almost all patients who are treated with these anti-EGFR drugs experience disease progression within 2 years [17]. Although patients initially show favorable clinical responses, about 50-60\% rapidly develop secondary EGFR mutations and lose the sensitivity of EGFR TKIs.

On the other hand, the American Cancer Society (ACS) estimates that ALK-EML4 gene fusions are present in about 2-7\% of NSCLC patients. In previous clinical trials, it was found that patients who harbor ALK rearrangements did not benefit from therapy with EGFR TKIs. Subsequently, this led to the development and approval in 2011 of crizotinib, an oral ALK inhibitor that produced response rates of up to 57\% in ALK-positive cases. Thereafter, ceritinib and alectnib, both of which are more potent ALK inhibitors than crizotinib, have been approved for the treatment of ALK-positive NSCLC, especially in patients with tumors that are resistant to crizotinib. In addition to EGFR and ALK targeting drugs, other targeted therapies against HER2 mutations (trastuzumab), BRAF (dabrafenib), 
RET rearrangements (cabozantinib) and angiogenesis (bevacizumab) are also approved for the treatment of advanced NSCLC [17, 25, 26].

The most recent breakthrough therapeutic strategies for the treatment of advanced NSCLC are the immunotherapies. These new anticancer agents are mainly premised on the restoration of dysregulated immune system responses against cancer cells in patients [27, 28]. Unlike chemotherapy, immunotherapies indirectly target tumor cells by stimulating the individual patient's immune system mechanisms to attack tumors [6]. In general, there are two approaches to immunotherapy of cancer namely, (1) the vaccination and (2) the immune-checkpoint approaches. In the vaccination approach, antigens or cells are used to stimulate T-cells to eliminate tumor cells. Today, vaccines against EGF, TGF $\beta$, and PD-1 are at different stages of advanced clinical testing with promising findings and their possible introduction to the therapeutic armamentarium against NSCLC in the near future [4]. On the other hand, the immune-checkpoint approach utilizes specific monoclonal antibodies to neutralize specific tumor molecular pathways [4, 14]. The two most prominent drug targets for immune checkpoint inhibitors are: the cytotoxic T-lymphocyteassociated antigen-4 (CTLA-4) and programmed cell death protein-1 (PDL-1). In 2015, pembrolizumab and nivolumab, both of which are monoclonal antibodies, received FDA approval for the treatment of metastatic NSCLC. Based on the results of the landmark KEYNOTE clinical trials, it has been determined that pembrolizumab is highly effective in patients expressing PDL-1 on greater than $50 \%$ of tumor cells. Consequently, nivolumab and pembrolizumab have become the standard therapeutic strategy for patients with PDL1 overexpressing squamous and non-squamous advanced NSCLC. 
However, in the greater majority of NSCLC patients, tumors do not show targetable driver mutations. For these patients, the first-line systemic therapies are platinum-based combination chemotherapies [6]. These treatments are administered as two or more drug combinations consisting of a platinum compound (such as cisplatin or carboplatin) alongside a second or third-generation chemotherapeutic agents. Usually, based on the patient's clinical status, four to six cycles are administered while monitoring the clinical response and toxicity profile. Today, the most common antitumor agents used alongside the platinum drugs are: paclitaxel, docetaxel, gemcitabine, pemetrexed or vinorelbine [18, 23]. These various drug combinations have been found to show similar clinical efficacies and the choice of treatment is mainly dictated by patient factors and toxicity profile [6, 24]. Clinically, it appears that the cisplatin-taxane therapies although are associated with greater toxicity potential, have consistently provided higher clinical response rates. In many treatment guidelines, a two-drug combination of cisplatin and paclitaxel are recommended as the standard regimen for stage IIIB-IV lung cancer with 1-year survival rate exceeding $40 \%[4,24]$. More recently, because of the lower toxicity profile of gemcitabine, a threedrug combination (in addition to cisplatin and paclitaxel) has also become popular but with minimal increments in overall patient survival [24]. In patients with non-squamous carcinoma, pemetrexed is recommended as the third drug to the cisplatin-paclitaxel combination and survival rates are between 13-14 months [6].

In summary, the targeted drugs and immunotherapies have revolutionalized the systemic therapies for NSCLC. However, platinum-taxane combinations still represent the the gold standard in the treatment for advanced NSCLC. Unfortunately, these drugs also represent a major pathway towards the development of chemoresistance and metastasis. 


\section{Withaferin A}

Withaferin A (WFA) is a member of a large group of naturally occurring 28 carboncontaining compounds collectively called the withanolides [19]. These withanolides (Fig. 1.2) are structurally composed of a steroidal framework to which a lactone ring is attached and are sometimes referred to as 'steroidal-lactones' [20]. The steroidal part of withanolides consists of network of 4-cycloalkane rings, 3-cyclohexane rings, and 1cyclopentane ring. The second part of withanolides, the lactone ring, is a cyclic ester which is appropriately oxidized to form a 6-membered ring [19]. This overall basic withanolide structure can be modified either in the steroid framework or the side chains to yield a myriad of compounds. As such, more than 300 different withanolides have been isolated and characterized [21]. Of all these compounds, WFA is the most potent and was the first member of the withanolides to be isolated from the Solanaceae species.

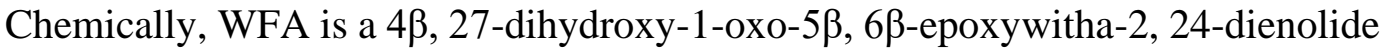
and was first isolated from alcoholic extracts of the Indian Ayurvedic medicinal plant Withania somnifera [20, 22-24]. The isolation of WFA from W. sominfera was prompted mainly by the systematic scientific review of the antitumor activities of Indian Ayurvedic medicinal plants of which W. somnifera, which is also known as Ashwagandha, or Indian ginseng is a popular medicinal plant in the Ayurvedic system. Traditionally, the different parts of W. somnifera are used to prepare various types of herbal formulations. Several preclinical studies were conducted demonstrating the anticancer effects extracts of $W$. somnifera (L.). Most notably, Devi et al., showed that intraperitoneal (i.p.) administration of alcoholic root extracts of $W$. somnifera significantly inhibited the growth of sarcoma180 xenografts in nude mice [23]. In addition, studies also demonstrated the inhibitory 
effects of W. somnifera on chemically-induced fibrosarcoma and skin tumors in Balb/C mice [25]. These studies provided the rationale and fueled the investigation and isolation of the bioactive constituents of $W$. somnifera, which were found to be the withanolides. In 1965, the first and major withanolide, WFA was isolated from alcoholic extracts of the leaves of W. somnifera. Later, WFA was isolated from other species such as Withania aristata, Dunalia spinose and Vassobia breviflora [21, 24].

Following the isolation of WFA as a crystalline compound alongside its dihydroderivative from leaves of $W$. somnifera, its partial and final chemical structures of WFA were first solved by Lavie et al., [20, 23, 24]. In its basic chemical structure (Fig 1.2), WFA is composed of an ergostane framework to which a lactone ring is attached. The lactone ring in WFA is a cyclic ester composed of 5-carbon atoms, in which the carbon atoms at C-22 and C-26 are oxidized to form a 6-membered ring [26]. This unique arrangement of atoms in the WFA structure results in five chemically active functional groups. These groups include; the unsaturated ketone ring, a hydroxyl group at C-4, epoxide ring between C-5 and C-6, a hydroxyl group at C-27, a 6-carbon lactone ring, and an unsaturated carbonyl group [27, 28]. Through these functional groups, WFA has the ability to interact with multiple molecular targets, particularly proteins, resulting in several pharmacological activities [22, 29]. Currently, review of the literature shows that WFA has antitumor, anti-inflammatory, antiangiogenic, cardioprotective, immunomodulatory, hepatoprotective, anticonvulsant and anabolic effects [21, 29]. In spite of these multiple effects, WFA has attracted significant testing mainly for its anticancer activity [22].

The anticancer activity of WFA has been a subject of interest for many researchers around the world. Today, several published studies have explored the antitumor efficacy 
of WFA using cell culture, xenografts and orthotopic tumor models of multiple human cancers $[22,28-30]$. In most of these studies, WFA has been found to possess proapoptotic, cytotoxic, anti-invasive and anti-migratory effects against multiple tumor cell types. Mechanistically, WFA regulates several molecular targets in tumor cells including enzymes, signaling molecules and other proteins crucial to the survival of cancer cells [19, 29]. Results from structure-activity studies have consistently demonstrated that three positions in the WFA structure are critical for its interaction with sulfhydryl groups of cysteine residues of proteins [27]. These three positions include; (1) the unsaturated ketone (C-3) in the A-ring, (2) the epoxide group at C-5, and (3) the lactone ring [27].

Recently, spectral analysis of the WFA structure has revealed that C-3 position in the unsaturated A-ring is the main target for thiol group binding [31]. In fact, it has been hypothesized that the sulfhydryl groups of cysteine residues react with C-3 via 'MichaelAddition' alkylation type of reactions to form covalent bonds [28, 32]. These type of reactions are thought to play a major role in the ability of WFA to inhibit several signaling molecules in cancer cells. Furthermore, other studies have demonstrated that the epoxide ring between C-5 and C-6 is the most critical for the cytotoxic anticancer effects of WFA. For example, in vitro studies have shown that the reaction of WFA and 2-mercaptoethanol, which specifically affects the 5 $3,6 \beta$-epoxide structure, resulted in the loss of anticancer activity. Similarly, the incubation of WFA with strong reducing agents like N-acetyl cysteine (NAC) and dithiothreitol (DTT) resulted in the loss of the pro-apoptotic activity of WFA [33]. In other studies, it has been reported that the loss of unsaturation in the Aring caused loss of anti-inflammatory and antiangiogenic effects of WFA [21, 34]. 
In the preclinical setting, several studies have demonstrated the antitumor effects of WFA against various cancer types. Briefly, there is evidence to show efficacy of WFA against melanoma, breast, lung, prostate, ovarian, thyroid and gastrointestinal cancers. Perhaps, of all these, the most extensively investigated type of cancer for which WFA has shown excellent activity is breast cancer. For this canvcer type, multiple lines of evidence using different tumor models from independent studies indicate that WFA is proapoptotic in estrogen-sensitive (MCF-7) and estrogen-independent (MDA-MB-231) breast cancer cells in vitro [35]. To corroborate these in vitro findings, in vivo studies conducted using nude mice have reported statistically significant inhibition of the growth of subcutaneous MDA-MB-231 xenografts. Similarly, WFA has also attenuated the growth and metastasis of orthotopically implanted $4 \mathrm{~T}$ mouse mammary tumor cells [31, 36]. Furthermore, using clinically-relevant transgenic animal models for breast cancer, WFA reportedly inhibited more than 50\% of palpable tumor growth in MMTV-neu mice [36, 37].

In addition to breast cancer, preclinical efficacy for WFA has been demonstrated against prostate, ovarian, and cervical cancers. For example, intraperitoneal administration of WFA (4-8 mg/kg/day) for 24 days resulted in over 70\% inhibition of PC3 subcutaneous tumor growth in mouse models [38]. Here, the inhibition of tumor growth by WFA was attributed to the inhibition of proteasomal activity and subsequent induction of apoptosis. In another independent study, 3-5 mg/kg of WFA administered orally to transgenic mice for 39 weeks, inhibited tumorigenesis and metastasis of prostate adenocarcinoma [39]. Also, WFA alone or in combination with other drugs has shown excellent efficacy against cervical cancer and ovarian cancers. Data from in vitro and in vivo studies indicate that WFA has the capability of inhibiting the growth of human cervical cancer cells. It was 
found out that the antitumor activity of WFA in cervical cancer cells was related to the downregulation of the human papillomavirus oncogenes E6/E7, resulting in the induction of p53 and apoptosis [40]. More recently, the intraperitoneal administration of WFA alone or as a combinatorial agent alongside cisplatin to nude mice bearing A2780 human ovarian cancer cell xenografts resulted into $70-80 \%$ reduction in tumor growth and complete inhibition of metastasis [41-43]. Together, these data demonstrate the potential efficacy of WFA against gynecological cancers and further highlight the potential of WFA as a potential chemotherapeutic agent.

To date, the molecular mechanisms of action underlying the antitumor activity of WFA remain elusive. Nonetheless, there is a growing body of molecular mechanistic data which provides insights into the molecular targets of WFA in tumor cells. The actions of WFA can be summarized based on four (4) major activities of WFA in tumor cells. These include (1) proapoptotic effects, (2) antiproliferative activities, (3) antimetastatic and antiinvasive effects, and (4) cancer stem cell targeting effects. It has been shown that the proapoptotic effects of WFA are mainly mediated via the reactive oxygen species (ROS)dependent mechanisms, endoplasmic reticulum (ER) stress-dependent mechanisms, and the regulation anti- and pro-apoptotic proteins in tumor cells. On the other hand, WFA's effects on cellular proliferation are mainly mediated via G2/M cell cycle arrest-dependent mechanisms, such as the regulation p21 and cyclin-dependent kinases. In summary, current available evidence shows that WFA is a multifactorial antitumor compound with the ability to interact with a myriad cellular targets. The most plausible hypothesis postulates that the antitumor effects of WFA are mediated by molecular mechanisms involving the interaction of WFA and cellular proteins via thiol oxidation. 


\subsection{Statement of goals}

The overall goal of this doctoral thesis was to investigate the therapeutic potential of WFA, a plant-derived steroidal lactone against NSCLC. Briefly, studies were designed to explore the preclinical antitumor efficacy, toxicity and pharmacokinetic properties of WFA. Two human NSCLC cell lines, H1299 and A549 were used to evaluate the in vitro and in vivo efficacy of WFA alone or in combination with standard chemotherapeutic drugs such as PAC and cis-Pt against the growth and proliferation of human NSCLC cell lines. In addition, a PAC-resistant (TR-A549) cell line was used to explore the potential of WFA for targeting drug resistance in NSCLC. Finally, the potential repeated-dosing toxicity and pharmacokinetic properties of WFA were investigated in male SD rats to evaluate the disposition characteristics of WFA. In order to accomplish these research goals, the following specific aims were explored;

$\underline{\text { Aim } 1 .}$. To determine the synergistic effects of WFA alongside PAC on the growth and proliferation of human NSCLC cells in vitro.

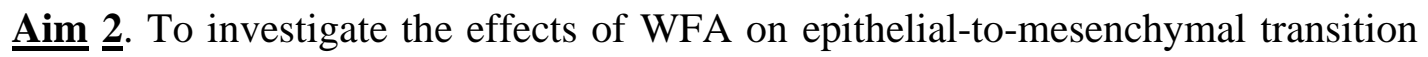
(EMT) and chemoresistance in human NSCLC cells

$\underline{\text { Aim 3. }}$. To determine the potential 28-day repeat-dose toxicity and the pharmacokinetics properties of WFA in SD rats.

Overall, the findings from the above studies advance a novel strategy of using WFA either alone or in combination with PAC as an effective strategy for targeting human NSCLC cells. These studies provide a strong rationale for conducting further studies to develop WFA as a clinically useful anticancer agent for the treatment of NSCLC and other cancers. 


\section{CHAPTER TWO}

\section{SYNERGISTIC COMBINATIONS OF PACLITAXEL AND WITHAFERIN A AGAINST HUMAN NON-SMALL CELL LUNG CANCER CELLS}

\section{INTRODUCTION}

Lung cancer remains the leading cause of cancer-related deaths among both men and women in the U.S [44]. This extremely poor prognosis is explained in part by three main characteristics of lung cancer: a) distant organ metastasis at diagnosis, b) a high degree of cellular and genetic diversity, and c) rapid development of drug resistance [15, 45]. Clinically, NSCLC represents $80-85 \%$ of all the cases of lung cancer and has an overall five-year survival rate of $15 \%[17,46]$. The therapeutic options for NSCLC are dependent upon the clinical stage at diagnosis [46-48] with surgical resection being the standard of care for early-stage NSCLC [49]. In early-stage NSCLC, the cure of the primary tumors is usually attainable in up to $80 \%$ and $50 \%$ for stage I and stage II cases, respectively. However, about $60-70 \%$ of the NSCLC cases are either locally advanced or extensively metastatic at the time of diagnosis $[5,13]$. Thus, systemic chemotherapy $[14,50]$ remains the cornerstone therapeutic strategy for the treatment of early-stage and advanced cases of NSCLC.

Decades of clinical trials have demonstrated that the use of chemotherapy relieves

disease symptoms and improves the quality of life in NSCLC patients [9]. Recently, landmark discoveries of driver genetic mutations have fueled the development and FDA 
approval of targeted $[9,51]$ and immunotherapies [52, 53]. Following the introduction of these treatments into the clinic, the frontline therapies for advanced NSCLC have been shifted and revolutionized towards an era of personalized medicine [12, 53, 54]. Yet, besides their high cost, the targeted drugs and immunotherapies only benefit small and specific groups of NSCLC patients. This is primarily true because less than $30 \%$ of all NSCLC patients show targetable mutations [55], and anti-PD-1 drugs are only effective among patients with tumors expressing PDL-1 on $>50 \%$ of tumor cells [56]. Therefore, despite these recent therapeutic advancements, NSCLC remains largely incurable, and the overall clinical benefit of current therapies in NSCLC is marginal and temporary [46, 48].

Platinum-based therapies are the first-line regimens used for neoadjuvant, adjuvant and in the palliative setting for advanced NSCLC [16, 57]. Upon diagnosis, NSCLC patients with no targetable driver mutations are usually administered with four-six cycles [57] of platinum-based chemotherapy. Two platinum compounds, cisplatin or carboplatin are used as the main drugs used to treat advanced NSCLC. These platinum compounds have nonspecific mechanisms of action [58] and induce apoptosis in both tumor and normal cells via the formation of DNA adducts. Mechanistically, cisplatin or carboplatin passively diffuses into cells and once inside the cell, one chloride ion is removed from the platinum compound and is replaced by a water molecule. Then, the hydrated platinum agent enters the nucleus, where it forms strong covalent bonds with purine bases, particularly guanine in DNA. The binding of the cisplatin or carboplatin with guanine residues creates intrastrand crosslinks, which cause extensive unrepairable DNA damage, and subsequently induce apoptosis. Of the two platinum drugs, cisplatin shows the highest antitumor efficacy but produces the most significant toxicity in the form of nephrotoxicity as well as the 
irreversible and often untreatable neurotoxicity. Although effective as monotherapies, platinum drugs are administered as combination chemotherapy alongside a thirdgeneration chemotherapeutic agent $[13,14,16,57]$.

The two-drug taxane-platinum chemotherapeutic combinations represent the standard of care as platinum-based systemic treatments for advanced NSCLC [9, 59]. Taxanes as a novel class of anticancer agents is a large group of compounds that target microtubules inhibiting spindle formation and blockade of mitosis during cell division [60]. Paclitaxel (PAC), the most prominent taxane, was first isolated from extracts of the bark of Taxus brevifolia (Pacific Yew Tree) in 1964. Unlike other tubulin inhibitors, PAC's mode of action [61] involves the binding to and preventing microtubule disassembly, thus causing mitotic arrest, and the induction of apoptosis. In NSCLC, PAC displays greater antitumor potency than cisplatin (cis-Pt) against all subtypes NSCLC [14]. Unfortunately, since PAC displays variable clinical pharmacokinetics, primarily due to its hydrophobicity, it requires large doses. Thus, although highly efficacious, the cis-Pt+PAC chemotherapy suffers dose-limiting toxicity and the clinical efficacy has plateaued at about $10-14$ months.

In the present study, we tested a novel therapeutic strategy to enhance the antitumor efficacy of PAC against NSCLC cells through combination with withaferin A (WFA). WFA (Fig. 1a), a member of the withanolides, is a plant-derived anticancer compound that was first isolated [23] from extracts of the Indian Ayurvedic medicinal herb, Withania somnifera (Ashwagandha). In the recent past, WFA has attracted significant preclinical testing [22] mainly for its antitumor activity against lung [40, 62-65], breast [31, 35, 37], uterine and cervix [66], ovarian [43], pancreatic [67], B-cell lymphoma [68]. In the present 
study, we have demonstrated that at various combinatorial ratios, WFA alongside PAC is highly synergistic against the growth and proliferation of human NSCLC cells. Specifically, our data indicated that PAC+WFA synergistically inhibited colony formation, migration, invasion and induced apoptosis in both drug-sensitive and drug-resistant human NSCLC cells. When combined, WFA caused a dramatic increase in the efficacy and potency PAC against both H1299 and A549 cells. Using the combination index (CI) and dose-reduction index values, we found PAC+WFA to be more synergistic than PAC+cisPt or cis-Pt+WFA. In fact, we achieved up to a 40 -fold reduction in the $\mathrm{IC}_{50}$ of $\mathrm{PAC}$ at a 1:40 constant ratio of PAC to WFA. In summary, this study demonstrates the potential therapeutic efficacy of WFA alone, and in combination with PAC against NSCLC cells and provides a strong rationale for advancing this combination in clinical trials. 


\section{MATERIALS AND METHODS}

\section{Drugs, chemical supplies, and reagents}

Withaferin A (purity, > 95\%) was provided as a gift sample by 3P Biotechnologies (Louisville, KY, USA), paclitaxel (Cat \# P-9600) was purchased from LC laboratories (Woburn, MA, USA), cisplatin (cis-diamine platinum [II] dichloride, Cat \# P4394) and 2.3\% crystal violet solution (Cat \# HT90132) were purchased from Sigma Aldrich (St. Louis, MO, USA). MTT (3-[4,5-dimethylthiazol-2-yl]-2,5-diphenyltetrazolium bromide, Cat \#L11939 ) was purchased from Alfa Aesar (Ward Hill, MA, USA) while dimethyl sulfoxide (DMSO, Cat \#13390) was purchased from Electron Microscopy Sciences (Hatfield, PA, USA). Dulbecco's Modified Eagle’s Medium (DMEM, Cat \#10569044), RPMI medium 1640 (Cat \# 11875-095), 0.25\% Trypsin-EDTA (Cat \# 25200-072), antibiotics (100 U penicillin/100 mg streptomycin, Cat\# 15140), and ultrapure distilled water (Cat \#10977-015) were purchased from Life Technologies (Grand Island, NY, USA). Heat-inactivated fetal bovine serum (FBS, Cat\# 12306C)) was purchased from SAFC (St. Louis MO, USA). Pierce RIPA cell lysis buffer (Cat \#89901), 100x halt protease/phosphatase inhibitor cocktail (Cat \#7844), Pierce BCA protein assay kit (Cat \#23225), PVDF-transfer membrane (Cat\#88518), Spectra Multicolor broad range protein ladder (Cat \#26634) and Pierce ECL Western Blotting Substrate (Cat \# 32106) were purchased from ThermoScientific (Rockford, IL, USA). Bolt 4-12\% Bis-Tris phosphate gels (Cat \#NW04125Box, NW04120Box, NW04122Box) and 20x Bolt MES running buffer (Cat\# B002) were purchased from Life Technologies (Carlsbad, CA, USA). The FITC-AnnexinV/PI Apoptosis Assay kit (Cat\#V13242) was purchased from Life Technologies (Eugene, OR, USA). Primary monoclonal antibodies (against $\beta$-actin, PARP, 
P21, Bc12, Bax, phospho-histone3, phospho-cdc2, cyclin $\mathrm{E}_{2}$, cyclin $\mathrm{B}_{1}$, cyclin A1, cleaved -caspase 3, cleaved-caspase-7, cleaved-PARP) as well as secondary antibodies (antimouse IgG and anti-rabbit IgG) were purchased from Cell Signaling Technology, Inc. (Danvers, MA).

\section{Cell lines and culture conditions.}

The human NSCLC cell lines, H1299, and A549 cells were obtained from American Type Culture Collection (Manasa, VA, USA). Previously developed [69, 70] PAC- resistant NSCLC cells (TR-A549) were kindly provided by Dr. Bruce Zetter (Boston Children's Hospital, Boston, MA, USA). These cell lines were maintained in either DMEM or RPMI 1640 culture media (10\% FBS, 1\% antibiotics) for A549 and H1299 cells, respectively. The cultures were incubated at $37^{\circ} \mathrm{C}$ in a $5 \% \mathrm{CO}_{2}$ humidified incubator and passaged at $\sim 80 \%$ confluence for less than 20 cycles.

\section{Drugs and treatments.}

Stock solutions of PAC, WFA, and cis-Pt at concentrations of $5 \mathrm{mM}$ were prepared by dissolving in $100 \%$ DMSO at $25^{\circ} \mathrm{C}$. The stock solutions for each drug were stored dried in aliquots of $40 \mu \mathrm{L}$ at $-20^{\circ} \mathrm{C}$ until used in subsequent experiments. Cells were incubated with the drugs after diluting the stock concentrations of each test agent in cell culture media to desired concentrations. In all treatments, DMSO was kept at a maximum of $1 \%$ in the vehicle and test treated groups.

\section{MTT cell viability assay.}

Cell viability was measured using the MTT colorimetric assay. Briefly, A549 or $\mathrm{H} 1299$ cells were seeded in $96-$ well plates and incubated at $37^{\circ} \mathrm{C}$ overnight to allow attachment. Following cellular attachment, the cell culture media was discarded and 
replaced with fresh cell culture media containing different concentrations of drugs/agents. These cells were incubated with the drugs for designated periods and then incubated with cell culture media containing $0.5 \mathrm{mg} / \mathrm{mL}$ MTT reagent solution. After 3 hours, the MTT containing media was discarded and the purple formazan crystals in each well were solubilized using $200 \mu \mathrm{L}$ of DMSO. The absorbance of the resulting solution was determined spectrophotometrically by measuring the optical density (OD) at $570 \mathrm{~nm}$. The OD values of the solution in the wells containing untreated cells were considered as $100 \%$ cell growth and used as a reference to calculate the percent growth of other wells. The arithmetic mean of 3 technical replicates was calculated at each concentration of PAC and WFA alone and in combination to obtain the percent cell viability. Data were expressed as mean \pm SD of more than 3 separate experiments.

\section{Colony formation assay}

To perform the colony formation assay, H1299 and A549 cells were seeded in 6well tissue culture plates at a density of 500 cells/well in $2 \mathrm{~mL}$ of medium (10\% FBS) and incubated in a $37{ }^{\circ} \mathrm{C}$ humidified atmosphere containing $5 \% \mathrm{CO}_{2}$ for $24 \mathrm{~h}$. The cells were then incubated in culture medium containing different concentrations of either PAC or WFA, alone and in combination for $24 \mathrm{~h}$. The drug-containing medium was replaced with fresh drug-free medium. After $7 \mathrm{~d}$, the plates were washed with sterile PBS, and the cells were fixed using methanol/acetic solution (3:1) for 5 mins and stained with $0.5 \%$ crystal violet (in methanol) for $15 \mathrm{~min}$. The crystal violet solution was carefully removed, the plates were rinsed in a stream of running water and left to air dry at room temperature. The number of colonies in each well was counted under a microscope. 


\section{Apoptosis analysis by flow cytometry.}

Apoptosis was detected using the Annexin V/ propidium iodide (PI) assay. Briefly, H1299 and A549 cells were treated with PAC, WFA alone, and in combination for $24 \mathrm{~h}$. Both floating and attached cells were collected, washed twice with ice-cold PBS and resuspended in $100 \mu \mathrm{L}$ of Annexin-binding buffer at a cell density of approximately $1 \times 10^{6}$ cells/mL. To each $100 \mu \mathrm{L}$ of cell suspension, $5 \mu \mathrm{L}$ of FITC-Annexin V and $1 \mu \mathrm{L}$ of 100 $\mu \mathrm{g} / \mathrm{mL}$ PI solution was added and incubated for 15 minutes in the dark. After incubation, $400 \mu \mathrm{L}$ of Annexin binding buffer was added to each Annexin V/PI stained cell suspension and analyzed for fluorescence using a flow cytometer by measuring the fluorescence emission at $530 \mathrm{~nm}$ and $>575 \mathrm{~nm}$. A total of 10,000 cells were counted in each cell suspension, and the data were analyzed using FlowJo software (Trista, CA, USA) to obtain the percent early and late apoptotic cells.

\section{Cell migration and motility assays.}

The wound-healing assay was performed to assess cell migration and motility. This was accomplished using the 2-well culture inserts (ibidi ${ }^{\circledR}$ ) following the manufacturer's instructions. Briefly, ibidi ${ }^{\circledR}$ culture inserts were placed in 6-well plates and $3 \times 10^{5}$ cells $/ \mathrm{ml}$ were seeded into each of the two chambers of the same insert. The cells were incubated in the inserts for 24-h to obtain confluent monolayers and the inserts were removed to create a wound area (gap) between the two cell growth areas. Floating and dead cells were removed by washing the cells twice using culture media, then media containing PAC or WFA alone and in combination was added. Cellular migration and motility were assessed using a bright-field microscope at 0 and $24 \mathrm{~h}$. The migrated cells were photographed using 
a microphotographic camera, and the cell-covered areas were measured using WimScratch software program (Wimasis, Munich, Germany).

\section{Transwell migration and invasion assay.}

Cell migration and invasion assays were performed using the transwell culture inserts that were pre-coated with or without $40 \mu \mathrm{L}$ of $3.0 \mathrm{mg} / \mathrm{mL}$ Matrigel (BD). H1299 cells $\left(4 \times 10^{4}\right)$ suspended in $200 \mu 1$ of the serum-free medium in the presence and absence of test agent(s) were seeded onto the upper compartment of the transwell chamber. The lower chamber was filled with $600 \mu \mathrm{L}$ of media supplemented with $10 \%$ FBS as an attractant to cause cell migration. After $24 \mathrm{~h}$ incubation, cells on the upper surface of the transwell insert were removed using a cotton swab. The migrated cells at the bottom of the insert were fixed using 4\% paraformaldehyde, permeabilized using $100 \%$ methanol and stained using $0.2 \%$ toluidine blue (in $1 \%$ sodium borate). The number of migrated cells were counted under the light microscope.

\section{Western blot analysis.}

Whole-cell protein lysates were prepared using RIPA buffer as per the manufacturer's instructions. The total protein concentration was determined using BCA assay and an aliquot of $20 \mu \mathrm{g}$ total cellular protein of each sample was resolved by SDSPAGE on $4-12 \%$ Bis-Tris gels. The separated proteins were transferred to PVDF membranes, and these membranes were blocked using 5\% non-fat dry milk in TBST for 1 h. To determine the relative expression of specific proteins, membranes were first probed with primary antibodies followed by the respective HRP-conjugated secondary antibodies. 
The expression levels of each protein were determined by visualizing the protein bands using the chemiluminescent Pierce ECL western blotting reagent.

\section{Tumor xenograft studies in nude mice}

Taxol resistant NSCLC cells (TRA549 cells) were used to determine the in vivo efficacy of WFA. Female athymic nude (nu/nu) mice (5-6-week old) were purchased from Charles Rivers Labs and were maintained in accordance with the Institutional Animal Care and Use Committee (IACUC) guidelines. Briefly, TR A549 cells $\left(2.5 \times 10^{6}\right.$ cells/mouse) in $100 \mu \mathrm{L}$ (50:50) of serum-free media mixed with Matrigel matrix (Becton Dickinson, Bedford, MA) were subcutaneously injected into the left flank of each mouse. The mice were provided purified AIN-93M diet and water ad libitum. Once the average tumor size reached about $80-120 \mathrm{~mm}^{3}$, the mice were randomly divided into 3 groups $(\mathrm{n}=5)$ and treated with vehicle, PAC (10 mg/kg divided in 3 doses per week) and WFA (10 mg/kg) as 3 doses per week. All treatments for the vehicle control and intervention drug groups were done via intraperitoneal injections (i.p) and the tumor volumes were measured twice weekly.

\section{Statistical analysis.}

Statistical analysis was performed using Graph Pad Prism 8.0 (La Jola, CA) and CalcSyn 2.0 (Biosoft, Cambridge, UK). Data are presented as means \pm SD of at least 3 separate experiments. To determine the synergistic interaction between PAC and WFA, the dose-effect data on percent cell viability was analyzed by the combination index (CI) method described by Chou et al.[71]. The CI values; $\mathrm{CI}<1, \mathrm{C}=1$ and $\mathrm{C}>1$ indicated synergism, additive and antagonism, respectively. 


\section{RESULTS}

\section{WFA inhibits the proliferation of NSCLC cells via thiol oxidation}

To determine the antiproliferative effects of WFA alone (Fig. 2.1a) on NSCLC cells, we first evaluated its in vitro antitumor effects on monolayers of two human NSCLC cell lines. Briefly, H1299 cells (large cell carcinoma) and A549 cells (adenocarcinoma) were seeded in 96-well plates (3000 cells/well) and incubated with WFA $(0-5 \mu \mathrm{M})$ for 3 - $72 \mathrm{~h}$. The relative cell viability of both cell lines was measured after $72 \mathrm{~h}$ incubation by the MTT assay. By considering the vehicle treated cells as $100 \%$ cell viability, WFA significantly decreased the the viability of both H1299 and A549 cells in a dose- and timedependent manner (Fig. 2.1b and Fig 2.1c). The median inhibitory concentrations ( IC $\left._{50}\right)$ for WFA as calculated by median-effect equation method using CalcuSyn software were in the sub-micromolar range $(0.20-0.68 \mu \mathrm{M})$ for both $\mathrm{H} 1299$ and A549 cell lines. The efficacy (>90\% inhibition) for WFA was observed at when cells were incubated with WFA for $48 \mathrm{~h}$ and $72 \mathrm{~h}$. In either cell lines, the concentrations of WFA $\geq 2 \mu \mathrm{M}$ resulted in $>90 \%$ inhibition of cell proliferation at $48 \mathrm{~h}$ and $72 \mathrm{~h}$. To support the MTT data, we next examined whether WFA induced apoptosis in H1299 and A549 cells. Since we observed using the MTT assay that concentrations of WFA $\geq 2 \mu \mathrm{M}$ produced maximum efficacy, we chose this dose level to investigate the ability of WFA to induce apoptosis. The cells were incubated with or without $2 \mu \mathrm{M}$ WFA for $24 \mathrm{~h}$ and apoptosis induction was determined using Annexin-V/PI staining assay. Our data (Fig. 2.1d) indicated that WFA when compared to the vehicle-treated cells significantly increased the percentage of Annexin V positive cells, an indicator of induction of apoptosis. The induction of apoptosis was further confirmed by performing western blot analysis (Fig. 2.1e),where we found a dose-dependent increase 
in the activation (cleavage) of caspase-3, an increase in the expression of p21 and phosphoHistone 3 (p-H3), all of which were indicative of apoptosis.

Previously, studies have reported that the antitumor effects of WFA are dependent on the generation of reactive oxygen species (ROS) in tumor cells. Indeed, the role of ROS production as regards to the anticancer activity of WFA has been demonstrated in breast, ovarian and melanoma tumor cells. Similarly, to further investigate this hypothesis, we determined whether or not WFA induced the production of ROS in H1299 and A549 NSCLC cells. Briefly, cells were seeded in 6-well plates and incubated with $2 \mu \mathrm{M}$ WFA for $12 \mathrm{~h}$. The generation of ROS was qualitatively determined by fluorescence microscopy using H2DCFDA (Fig. 2.1f) and Mitosox Red (Fig. 2.1h) assays per manufacturer's instructions. Here, we found that the incubation of cells with WFA $(2 \mu \mathrm{M})$ increased the fluorescence intensity of H2DCFDA (green) and Mitosox Red (red) in both H1299 and A549, an indicator of increased production of ROS (Fig. 2.1f and Fig. 2.1h). In this study, hydrogen peroxide $\left(\mathrm{H}_{2} \mathrm{O}_{2}, 100 \mu \mathrm{M}\right)$ was used as a positive control. Interestingly, for both cell lines, the concomitant treatment of cells with WFA alongside with $\mathrm{N}$-acetyl cysteine (2.5 mM) significantly inhibited the production of ROS. To further provide perspective on the role of ROS production in the antitumor activity of WFA, we cultured both H1299 and A549 cells with WFA in the presence of 3 known ROS quenchers; N-acetyl cysteine (NAC), dithiothreitol (DTT) and Trolox. Interestingly, as has been reported elsewhere, the thiol-containing compounds NAC $(2.5 \mathrm{mM})$ and DTT $(100 \mu \mathrm{M})$ completely abrogated the anticancer activity of WFA against both $\mathrm{H} 1299$ and A549 cells. Contrastingly, Trolox, a non-thiol- containing ROS quencher did not inhibit the antiproliferative activities of WFA (Fig. 2.1g). 
a

Withaferin A

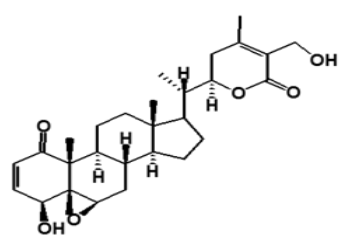

d

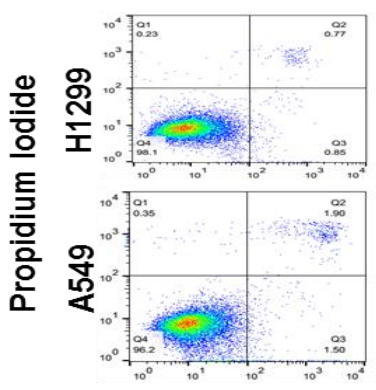

Annexin $V$ b

H1299

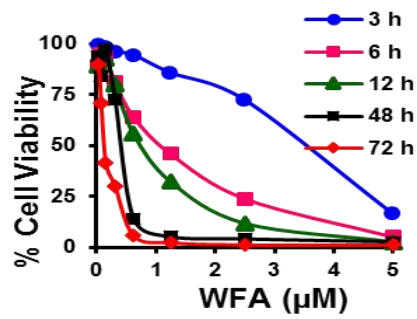

A549

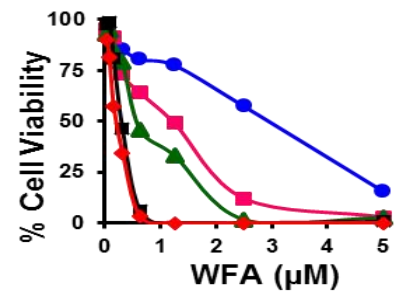

e

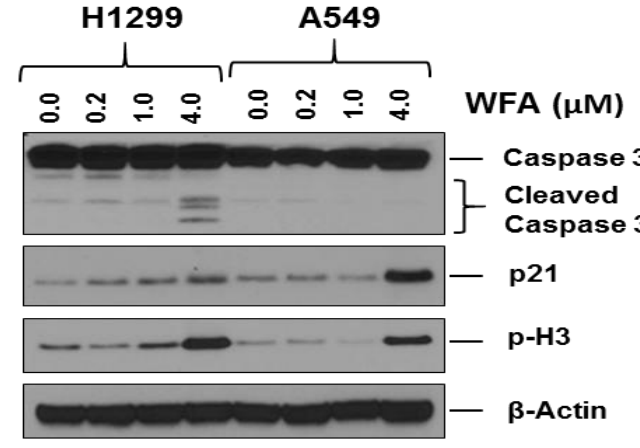

g
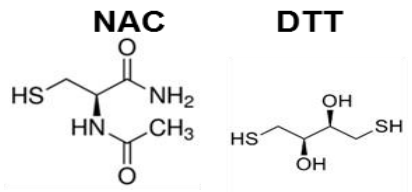

Trolox
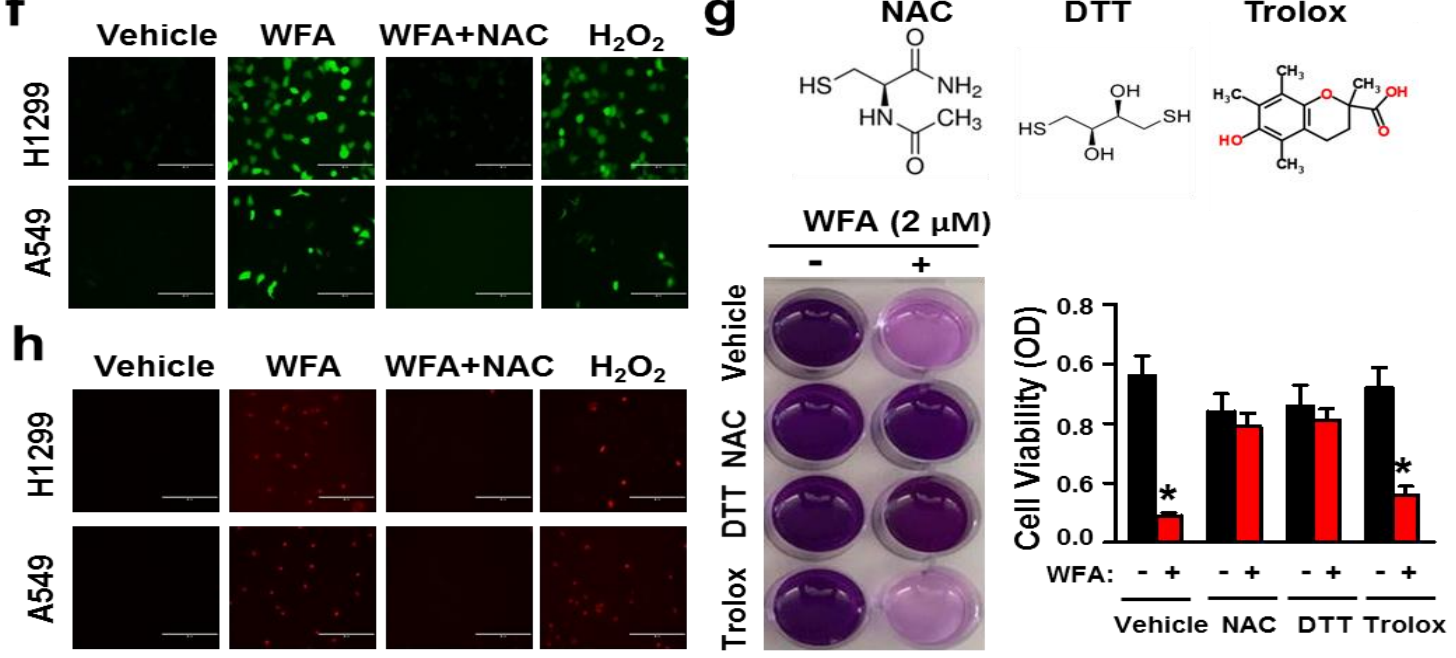

Fig 2.1. WFA inhibits the growth and proliferation of H1299 and A549 NSCLC cells via thiol dependent induction of apoptosis. (a) The chemical structure of WFA. The cells were incubated with WFA $(0-5 \mu \mathrm{M})$ for $3,6,12,48$ and $72 \mathrm{~h}$ and the cell viability measured by MTT assay. WFA time and dose-dependently inhibited the proliferation of H1299 (b) and A549 cells (c). The induction of apoptosis following incubation with WFA $(2 \mu \mathrm{M})$ was determined using annexin V/PI assay (d) and western blot analysis (e). Briefly, WFA increased the percentage of annexin V positive cells, the clevage of caspase 3 , and the expression of $\mathrm{p} 21$ and $\mathrm{p}-\mathrm{H} 3$. The generation of ROS was measured the intensity of green fluorescence in the H2DCFDA assay (f) and red fluorescence in the Mitosox Red assay (h). $\mathrm{H}_{2} \mathrm{O}_{2}(100 \mu \mathrm{M})$ was used as a positive control and N-acetyl cysteine (NAC) was used to inhibit ROS. (g) The antiproliferative activity of WFA was inhibited in the presence of thiol donors NAC $(2.5 \mathrm{mM})$ and dithiothreitol (DTT) but not in the presence Trolox. Where indicated, data are presented as mean \pm SD of 3 technical replicates $(* \mathrm{p}<0.05)$. 


\section{Synergistic effects of PAC, CisPt, and WFA on NSCLC cellular proliferation}

In this study, we determined the potential synergistic effects of PAC, cis-Pt, and WFA in H1299 and A549 cells in vitro. Firstly, we tested each of these agents as individual drugs and then based on the relative potency of each compound in the preliminary findings, we evaluated synergism using the following combinatorial ratios: 1:40, 1:100, and 10:1 of PAC: WFA, PAC:cis-Pt, and cis-Pt: WFA, respectively. In H1299 cells, we demonstrated that as individual drugs, PAC, cis-Pt, and WFA each dose-dependently inhibited the cellular viability (Fig. 2.2a). By using median-effect plots (Fig. 2.2b), we determined and compared the efficacy and potency of the three agents. We found that PAC displayed the greatest potency $\left(\mathrm{IC}_{50}: 43 \mathrm{nM}\right)$, followed by WFA $\left(\mathrm{IC}_{50}: 251 \mathrm{nM}\right)$ and then cis-Pt $\left(\mathrm{IC}_{50}\right.$ : $8438 \mathrm{nM}$ ). Based on these data, we next assessed whether the combinations of each drug with one of the other two compounds were synergistic against. Using the dose-response data (Fig. 2.2d-f) and isobologram analyses (Fig. 2.2g-i), we found that the data points for; PAC+WFA, PAC+cis-Pt, and cis-Pt+WFA were all below the lines of additivity at $\mathrm{IC}_{50}$, $\mathrm{IC}_{75}$, and $\mathrm{IC}_{90}$. In addition, visual inspection of CI-Fa plots (Fig. 2.2j-1) showed that the CI values at various effect levels were all $<1$. Therefore, as per the method of Chou et al., the dose-response data, isobologram and CI values all indicated that three combinations; PAC+WFA, PAC+cis-Pt, and cis-Pt+WFA were highly synergistic against H1299 cells,. However, based on the dose-reduction index (DRI), we found that there was a 33-fold and 4-fold reduction in the $\mathrm{IC}_{50}$ of PAC against $\mathrm{H} 1299$ cells in the PAC+WFA and PAC+cisPt combinations, respectively. On the other hand, we found a 26-fold and 7-fold change in the $\mathrm{IC}_{50}$ of cis-Pt when combined with WFA and PAC, respectively. For WFA, the $\mathrm{IC}_{50}$ were 5-fold and 8-fold less when combined with PAC and cis-Pt, respectively. 
Similarly, in A549 cells (Fig. 2.3), we also observed that PAC, cis-Pt, are WFA all individually decreased cell viability in a dose-dependent manner. Based on the medianeffect plots (Fig. 2.3b), PAC displayed the lowest $\mathrm{IC}_{50}(11 \mathrm{nM})$, followed by WFA (IC $\mathrm{I}_{50}$ : $560 \mathrm{nM}$ ) and then cis-Pt (IC 50: 5730). In terms of synergism (Fig. 2.3d-1), all combinations tested for the 3 drugs resulted in data points at $\mathrm{IC}_{50}, \mathrm{IC}_{75}$ and $\mathrm{IC}_{90}$ below the lines of additivity, indicating synergistic activity. As was observed in H1299 cells, the combination of PAC+WFA resulted in the strongest synergism (PAC DRI: 83-fold) followed by cisPt+WFA (cis-Pt DRI: 22-fold) and PAC+cis-Pt (6-fold and 15-fold DRI for PAC IC 50 and cis-Pt IC 50 , respectively). Together, these compelling in vitro data strongly suggested that the PAC+WFA combination was the most synergistic chemotherapeutic combination against the two human NSCLC cell lines, H1299and A549. 
a
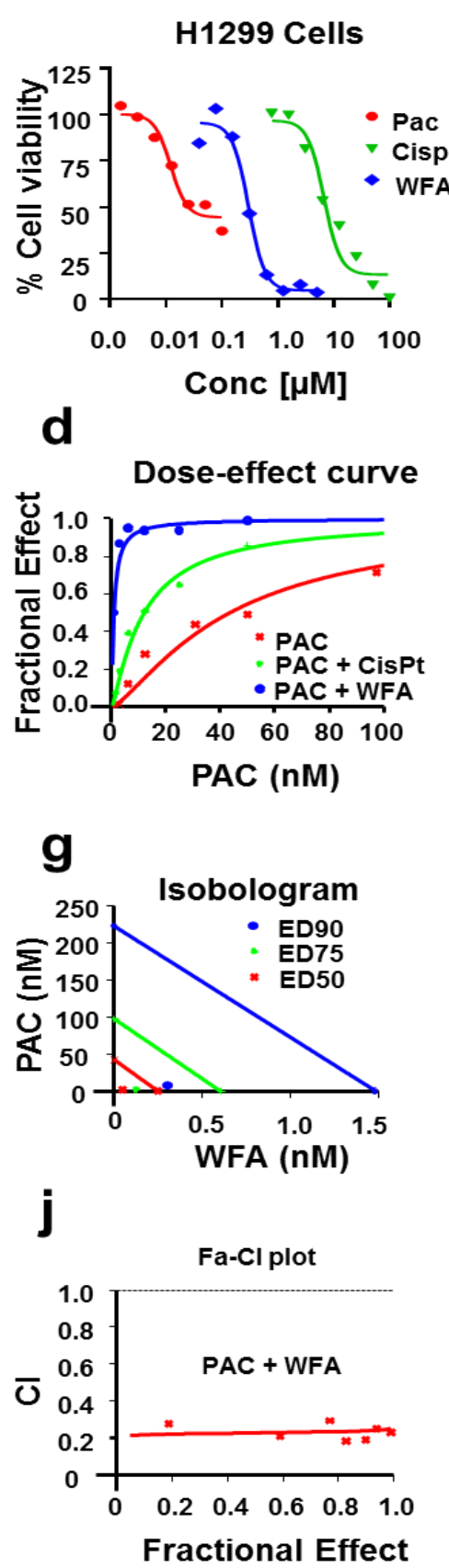

b

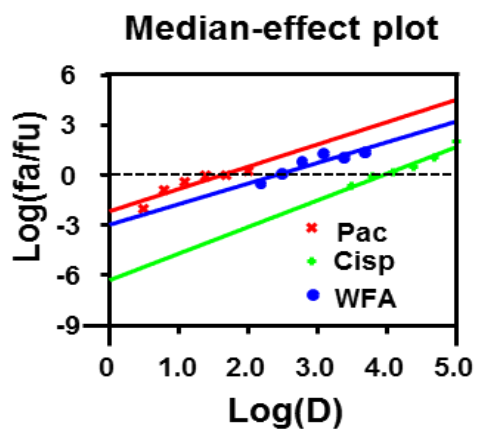

e

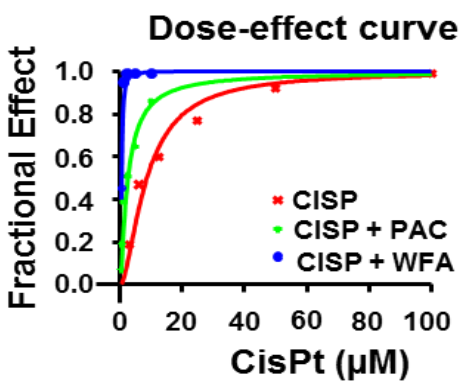

h

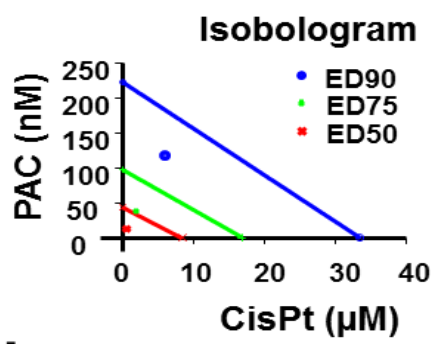

\section{$\mathbf{k}$}

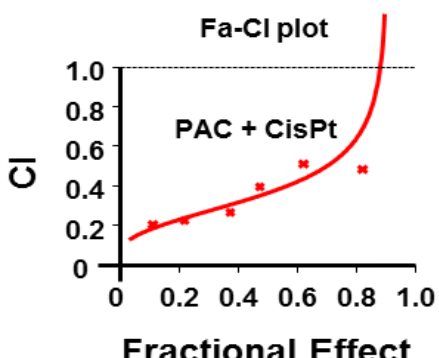

C

\begin{tabular}{lll}
\hline \multirow{2}{*}{ Drug } & \multicolumn{2}{c}{ Dose (nM) } \\
\cline { 2 - 3 } & IC $_{50}$ & \multicolumn{1}{c}{ IC $_{90}$} \\
\hline PAC & 43 & 223 \\
CisPt & 8438 & 33435 \\
WFA & 251 & 1500 \\
\hline
\end{tabular}

f

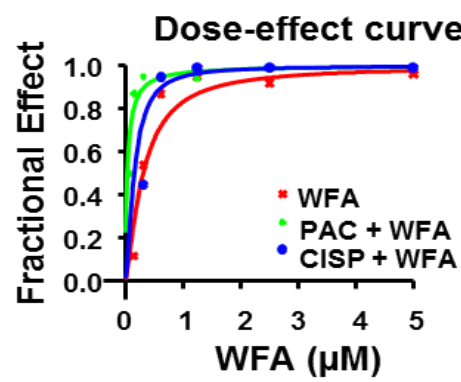

i

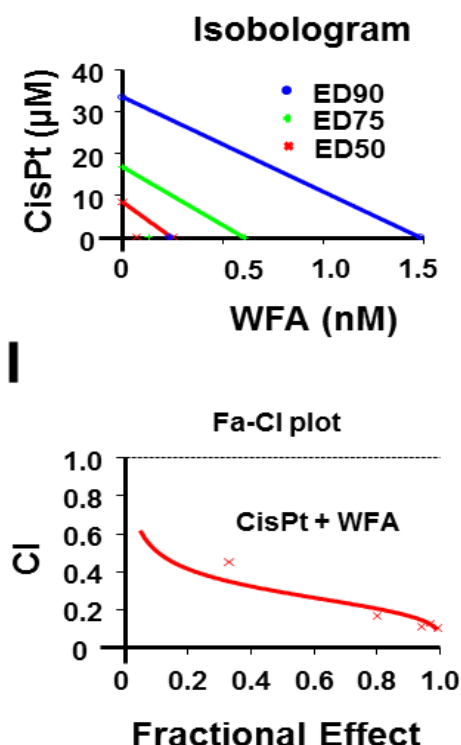

Fig 2.2. WFA synergized the antiproliferative activity of PAC and CisPt against $\mathrm{H1299}$ cells. The potency and efficacy of PAC, CisPt and WFA against NSCLC cells were compared using the median-effect equation. (a) Dose-response plots depicting PAC, CisPt and WFA dose-dependent inhibition of cell proliferation of H1299 cells. (b) Median-effect plot indicated PAC had lowest $\mathrm{IC}_{50}$ followed by WFA and CisPt the highest. Preliminary combinations of PAC and CisPt, PAC and WFA and CisPt and WFA were tested. PAC+WFA (c) displayed the greatest efficacy, followed by CisPt + WFA (d). Isobologram analysis $(\mathbf{g}-\mathbf{i})$ and combinaion index $(\mathrm{CI})$ values $(<1)$ indicated that all the combinations were highly synergistic against H1299 cells. 


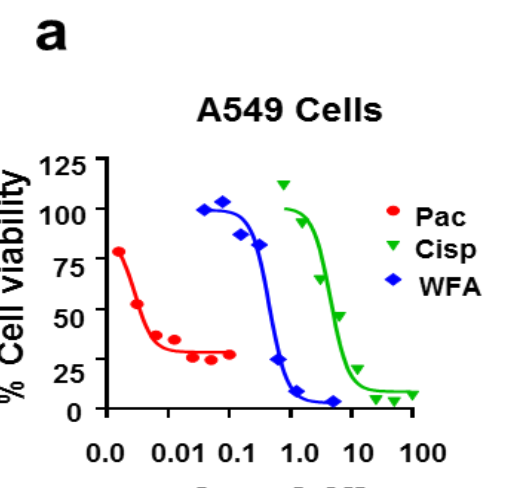

b

C

Conc $[\mu \mathrm{M}]$
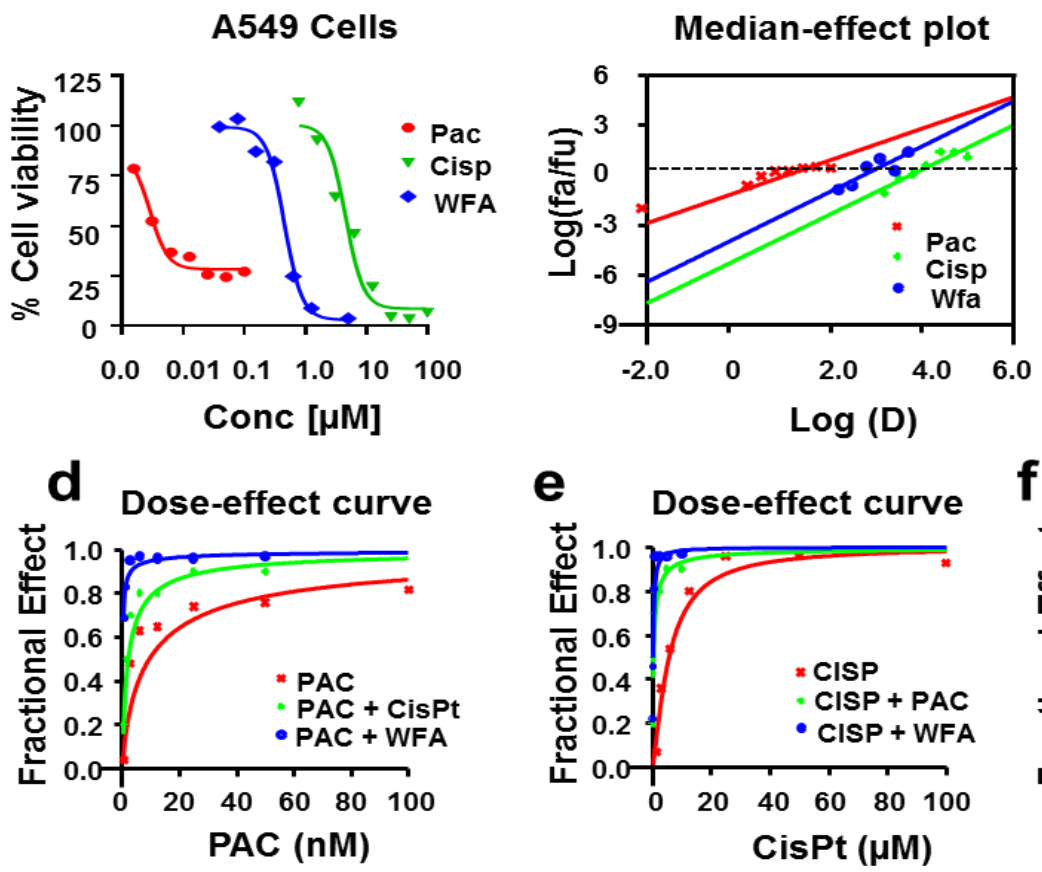

\begin{tabular}{ccc}
\hline \multirow{2}{*}{ Drug } & \multicolumn{2}{c}{ Dose (nM) } \\
\cline { 2 - 3 } & IC $_{50}$ & IC $_{90}$ \\
\hline PAC & 11 & 113 \\
CisPt & 5730 & 29460 \\
WFA & 560 & 2792 \\
\hline
\end{tabular}
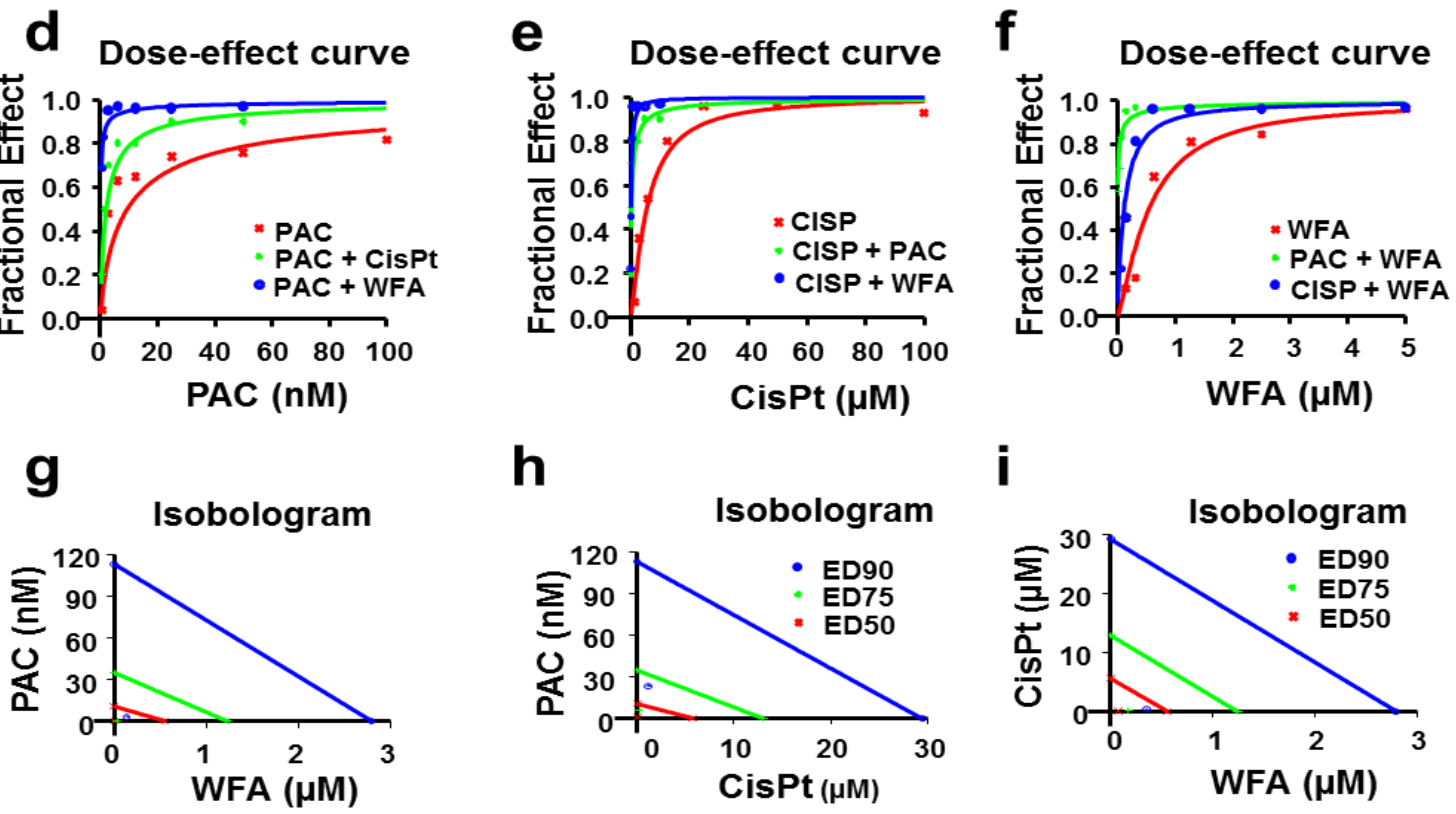

h

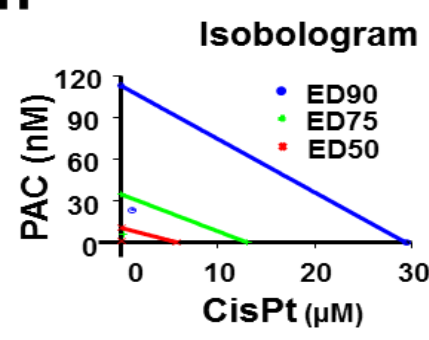

i

j

k

Fa-Cl plot

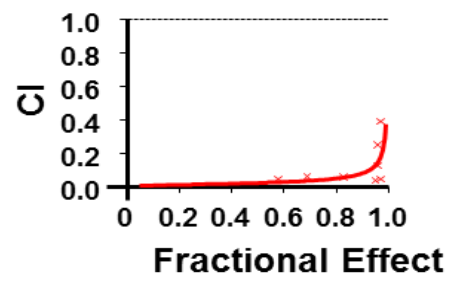

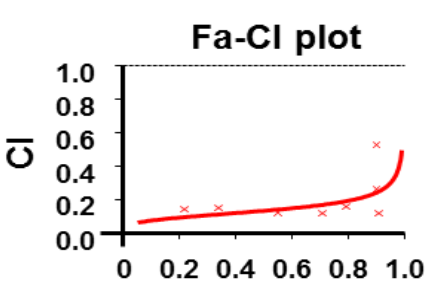

Fractional Effect

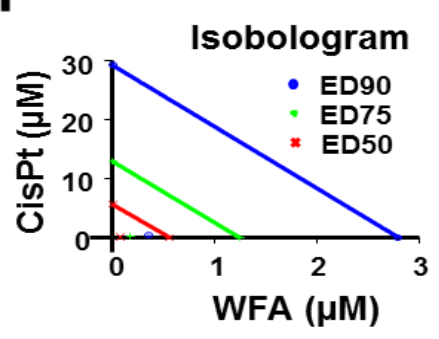

I

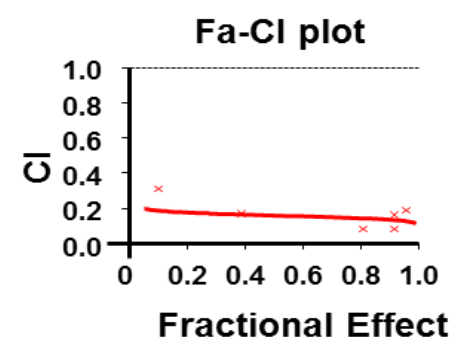

Fig 2.3. WFA synergized the antiproliferative activity of PAC and CisPt against A549 cells. The potency and efficacy of PAC, CisPt and WFA against A549 NSCLC cells were compared using the median-effect equation. (a) Dose-response plots depicting PAC, CisPt and WFA dosedependent inhibition of cell proliferation of A549 cells. (b) Median-effect plot indicated PAC had lowest $\mathrm{IC}_{50}$ followed by WFA and CisPt the highest. Preliminary combinations of PAC and CisPt, PAC and WFA and CisPt and WFA were tested. PAC+WFA (c) displayed the greatest efficacy, followed by CisPt + WFA $(\mathbf{d})$. Isobologram analysis $(\mathbf{g - i})$ and combinaion index $(\mathrm{CI})$ values $(<1)$ indicated that all the combinations were highly synergistic against A549 cells. 


\section{Synergistic effect of PAC and WFA on colony formation and induction of apoptosis}

Next, to validate the observed MTT data, we sought to investigate potential synergistic effects of PAC and WFA on the replicative ability of H1299 and A549 cells using colony formation assay (Fig. 2.4). Briefly, cells (500 cells/well) were seeded in 6well plates and incubated in media containing PAC (0-25 nM) and WFA (0-1 $\mu \mathrm{M})$ alone, or their combination. As expected, PAC and WFA individually displayed dose-dependent inhibition of colony formation in H1299 (Fig. 2.4a) and A549 (Fig. 2.4b) cells. As expected, the combination of PAC and WFA synergistically inhibited colony formation in both cell lines greater than either agent used alone. Our data (Fig. $2.4 \mathrm{c}-\mathrm{e}$ ), indicated that at shorter drug exposure durations resulted in much greater synergistic effects for PAC and WFA than was observed when cells were exposed to drugs for extended drug periods (48, $72 \mathrm{~h})$.

Further, we investigated whether the PAC + WFA combination induced apoptosis in NSCLC cells. Cells were incubated with WFA $(1.0 \mu \mathrm{M})$ or PAC $(25 \mathrm{nM})$, alone and in combination for $24 \mathrm{~h}$, and apoptosis was detected by flow cytometry using the Annexin V/PI assay. We found that the combinations of PAC and WFA significantly increased the percentage of Annexin- $\mathrm{V}$ positive cells (Fig. 2.4f). To support these findings, western blot was performed and the data obtained (Fig. 2.4g) indicated an increase in the cleavage of both PARP and caspase-3, Bcl-2 degradation, and Bax upregulation. In addition, p21 and phospho-H3 were found to be significantly increased, an indication of cell cycle arrest and subsequent induction of apoptosis. 


\section{The effect of different ratios, treatment schedules, sequence and cell numbers on the synergism of PAC and WFA}

As we found that PAC+WFA resulted in greater synergism than PAC+cis-Pt or cisPt+WFA against both H1299 and A549 cells, we further examined whether different combination ratios, cell numbers, sequences and schedules of treatment would alter the synergism. First, we used PAC as the standard of care and then altered the concentrations of WFA to test the effects of different ratios of PAC: WFA on cell viability. Based on previously obtained $\mathrm{IC}_{50}$ values, we tested combinations of PAC+WFA at 1:40, 1:20, and 1:10 ratios of PAC to WFA, respectively, on the viability of cells. For each of these ratios, we examined the resulting CI and DRI values to assess the extent of synergism. Using isobolograms, CI and DRI values, we found that all the ratios 1:40, 1;20, and 1:10 were synergistic against both H1299 and A549 cells. For all the combinatorial ratios, the CI values at 50\% (ED50), 75\% (ED75) and 90\% (ED90) effect levels were all <1 indicating synergism as per Chou et al.,. Since the 1:40 ratio of PAC: WFA produced the lowest combined $\mathrm{IC}_{50}$ against both $\mathrm{H} 1299(3.6 \mathrm{nM})$ and A549 $(7 \mathrm{nM})$, this combination was concluded to have displayed the greatest potency and efficacy of PAC and WFA. In terms of synergism, the highest synergistic interaction was displayed by the ratio of 1:10 of PAC:WFA since it resulted in the lowest CI values compared to 1:20 and 1:40 (Table 2.1 and Table 2.2).

Next, we determined the most effective strategy for combining PAC and WFA to maximize the synergism. We assessed the resultant synergism obtained when the cells were exposed to both drugs; (1) concomitantly, (2) sequentially, and (3) in a schedule-dependent manner. Firstly, and as expected, all the three treatment strategies (concomitant, sequential 
and schedule dependence) resulted in synergism of PAC and WFA against both H1299 and A549 cells (Table 2.3). Importantly, we found that by pretreatment of cells with WFA (2 h) prior to incubation with PAC+WFA, the resultant synergism $(\mathrm{CI}=0.22$ and 0.26 for H1299 and A549 cells, respectively) was greater than that observed when cells were pretreated with PAC prior to PAC+WFA $(\mathrm{CI}=0.87$ and 0.64$)$ or the simultaneous of PAC and WFA treatment (CI: 0.77 and 0.81). Interestingly, the synergism of PAC and WFA was not schedule-dependent as there were no significant differences in the potency and efficacy of either compounds when the drugs were added in a schedule-dependent manner. Furthermore, we determined and compared the CI and DRI values obtained when cells were incubated with PAC and WFA for various periods of time.

Further, we tested for the effects of PAC and WFA, alone and in combination on different cell numbers of H1299 and A549 cells. Briefly; 2000, 4000, 8000 and 12000 cells/well were seeded in 96-well plates and incubated with increasing concentrations of PAC and WFA, alone and in combination (Fig 2.5). The media containing drugs was discarded after $12 \mathrm{~h}$ and the cells were incubated for up to $48 \mathrm{~h}$ to determine cell viability. For both H1299 and A549 cell lines, there was a dramatic decrease in the individual efficacy of PAC or WFA with an increase in the number of cells plated. As expected, the lowest $\mathrm{IC}_{50}$ values for PAC and WFA were observed when incubated with 2000 cells/well (Fig 2.5a and Fig 2.5e). However, when the cell numbers were increased to 12000 cells/well (Fig 2.5d and Fig. 2.5h), there was up to 40-fold and 8-fold increase in the $\mathrm{IC}_{50}$ of PAC and WFA, respectively against both H1299 and A549 cells. Interestingly, while there was an increase in the combined $\mathrm{IC}_{50}$ values of PAC and WFA, there were no significant changes in the CI values at various cell numbers. Thus, we concluded that 
increasing the cell numbers of H1299 and A549 did not alter the synergism between PAC and WFA.

Table 2.1. Synergism Summary Table for PAC and WFA on H1299 Cells

\begin{tabular}{cccccccc}
\hline \multirow{2}{*}{ Test Agent } & \multicolumn{3}{c}{ Cl Values } & & Dm & m & r \\
\cline { 2 - 5 } & ED50 & ED75 & ED90 & & & \\
\hline PAC & N/A & N/A & N/A & 25 & 0.6 & 0.99 \\
WFA & N/A & N/A & N/A & 190 & 1.1 & 0.98 \\
PAC + WFA (1: 40) & 0.90 & 0.76 & 0.68 & 3.60 & 1.23 & 0.99 \\
PAC + WFA (1: 20) & 0.89 & 0.82 & 0.83 & 6.22 & 1.04 & 1.00 \\
PAC + WFA (1: 10) & 0.59 & 0.63 & 0.80 & 6.43 & 0.82 & 0.96 \\
\hline
\end{tabular}

Note: CI: Combination index, Dm: Median dose, m: shape of curve, r: goodness of fit

Table 2.2. Synergism Summary Table for PAC and WFA on A549 Cells

\begin{tabular}{cccccccc}
\hline & \multicolumn{3}{c}{ Cl Values } & & & \\
\cline { 2 - 4 } Test Agent & ED50 & ED75 & ED90 & Dm & m & r \\
\cline { 3 - 6 } PAC & N/A & N/A & N/A & 23 & 0.6 & 0.94 \\
WFA & N/A & N/A & N/A & 613 & 1.8 & 0.98 \\
PAC + WFA (1: 40) & 0.80 & 0.66 & 0.68 & 7 & 1.5 & 0.94 \\
PAC + WFA (1: 20) & 0.47 & 0.55 & 0.86 & 6 & 0.9 & 1.00 \\
PAC + WFA (1: 10) & 0.55 & 0.52 & 0.71 & 9 & 0.9 & 0.99 \\
\hline
\end{tabular}

Note: CI: Combination index, Dm: Median dose, $\mathbf{m}$ : shape of curve, $\mathbf{r}$ : goodness of fit 

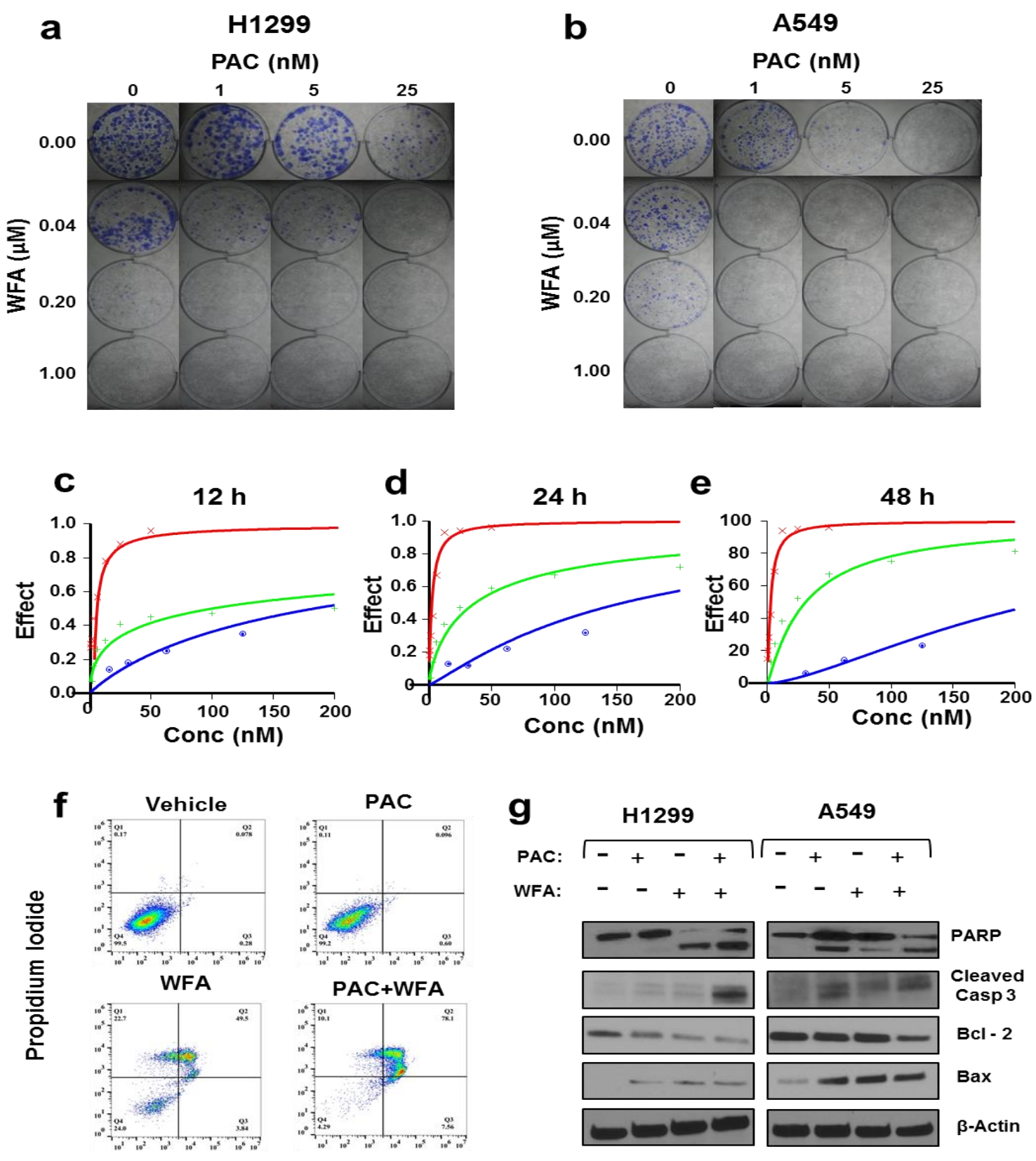

Annexin V

Fig 2.4. The combination of PAC and WFA significantly increased efficacy against colony formation, proliferation and induction of apoptosis in NSCLC cells. Representative images showing the colony formation assay in $\mathrm{H} 1299$ (a) and A549 (b) cells incubated with PAC and WFA alone and in combination. In both cell lines, PAC and WFA alone dose-dependently inhibited colony formation. However, the combination of PAC and WFA resulted in much greater inhibition of colony formation than either drug alone. Cells were also treated with PAC and WFA for $12 \mathrm{~h}$ (c), $24 \mathrm{~h}(\mathbf{d})$ and $48 \mathrm{~h}(\mathbf{e})$ and it was established that the synergism between PAC and WFA was greatest at the $12 \mathrm{~h}$ incubation time point. (f) Annexin V/PI assay and (g)western blot analysis depicted increased apoptosis induction. Moreover, the combination of PAC and WFA increased the cleavage of PARP, caspase 3, increased Bax and decreased the expression of Bcl2. 
a H1299 e

e

A549

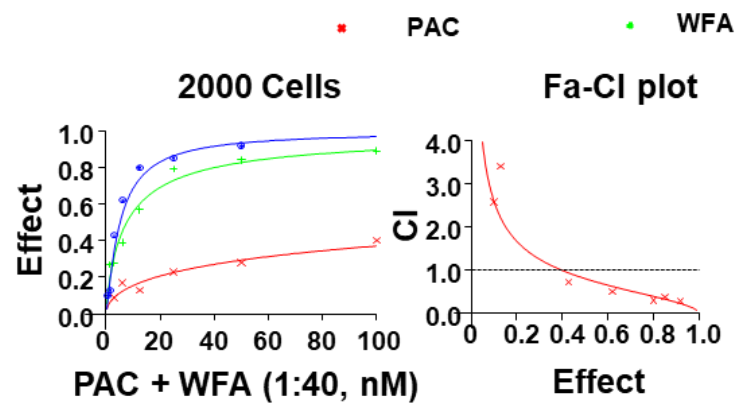

\section{Cells}

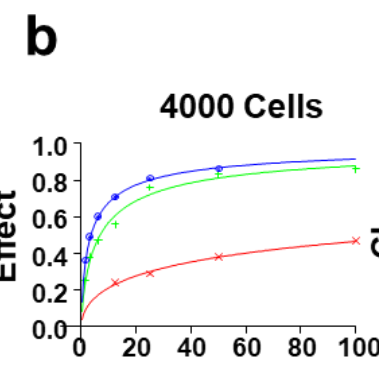

PAC + WFA (1:40, nM)
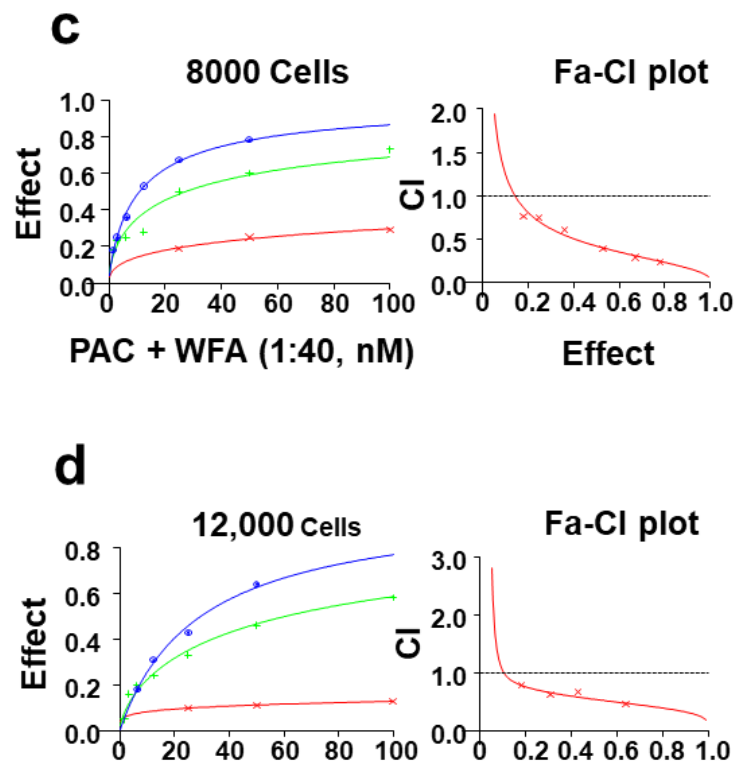

PAC + WFA (1:40, nM)

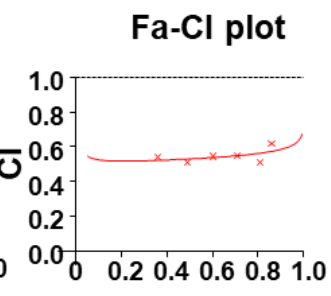

Effect
- PAC+WFA 2000 Cells
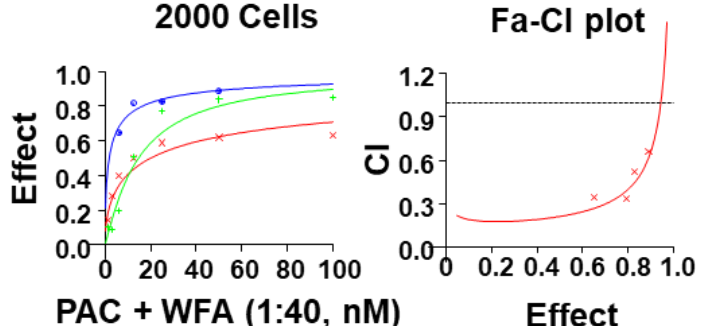

PAC + WFA $(1: 40, n M)$

f

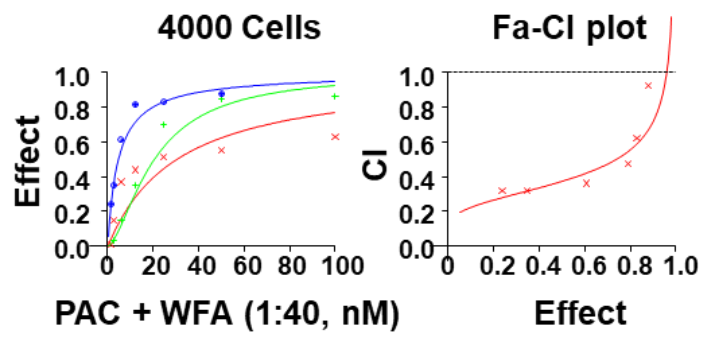

\section{g}

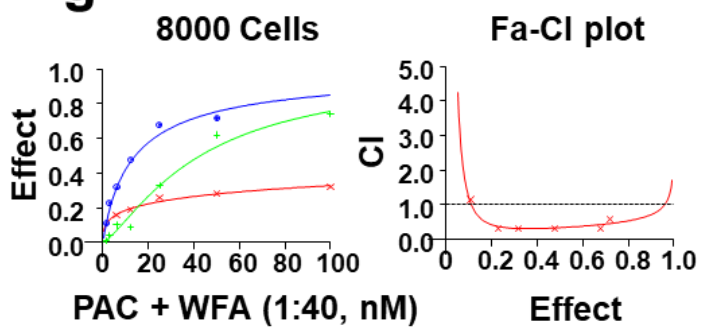

h

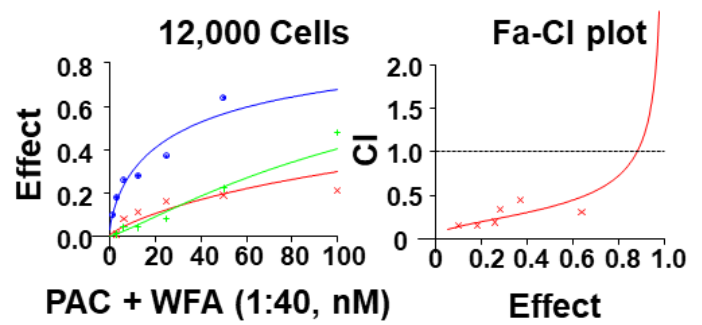

Fig 2.5. Increasing cell numbers did not alter the synergism of PAC and WFA against NSCLC cells. H1299 and A549 cells were plated at indicated densities and incubated with PAC and WFA alone, and in combination for $48 \mathrm{~h}$. The efficacy and potency of PAC and WFA alone was greatly diminished with increasing cell number in both H1299 (a-d) and A549 cells (e-h). However, the combination of PAC and WFA synergistically inhibited the proliferation of both cell lines regardless of the number of cells plated. Cell viability was measured by MTT assay, fractional effects and synergism were assessed by combination index $(\mathrm{CI}<1)$ as per as per Chou et al. 


\section{Effect of the PAC+WFA combination on migration and invasion of NSCLC cells.}

Cell motility, migration, and invasion are all important steps that are critical for the metastatic dissemination of NSCLC cells. Therefore, we investigated the effects of PAC and WFA, alone and in combination, on these cellular events. To determine effects on cell migration, we used the wound healing assay to assess the effects of PAC and WFA on cell motility into cell-free areas. Representative images (Fig 2.6)were obtained using a light microscope to monitor the wound areas between 0 and $24 \mathrm{~h}$ in order to determine the rate of cell motility. Low doses of PAC and WFA alone, inhibited the motility of H1299 (Fig. 2.6a) and A549 (Fig. 2.6b) cells. Comparatively, H1299 cells displayed greater migratory potential (>90\% migration in $24 \mathrm{~h}$ ) than A549 cells, thus the greatest effects on cellular migration for PAC and WFA were observed with A549 cells than H1299 cells. In both cell lines, at the tested concentrations, WFA displayed greater inhibitory effects on cell motility than PAC. However, the combination of PAC and WFA resulted in much greater inhibition

of cellular motility than either PAC or WFA alone $(<10 \%, * * \mathrm{p}<0.01)$. Furthermore, using the trans-well migration and invasion assays, we showed that PAC or WFA alone decreased migration and invasion compared to vehicle alone (Fig. 2.6c). As was observed in the motility assay, the combination of PAC and WFA also significantly inhibited cell migration than either drug used individually. In summary, the combination of PAC and WFA not only synergistically inhibited cellular growth and proliferation, but also significantly inhibited the migratory and metastatic capacities of H1299 and A549 cells. 
a
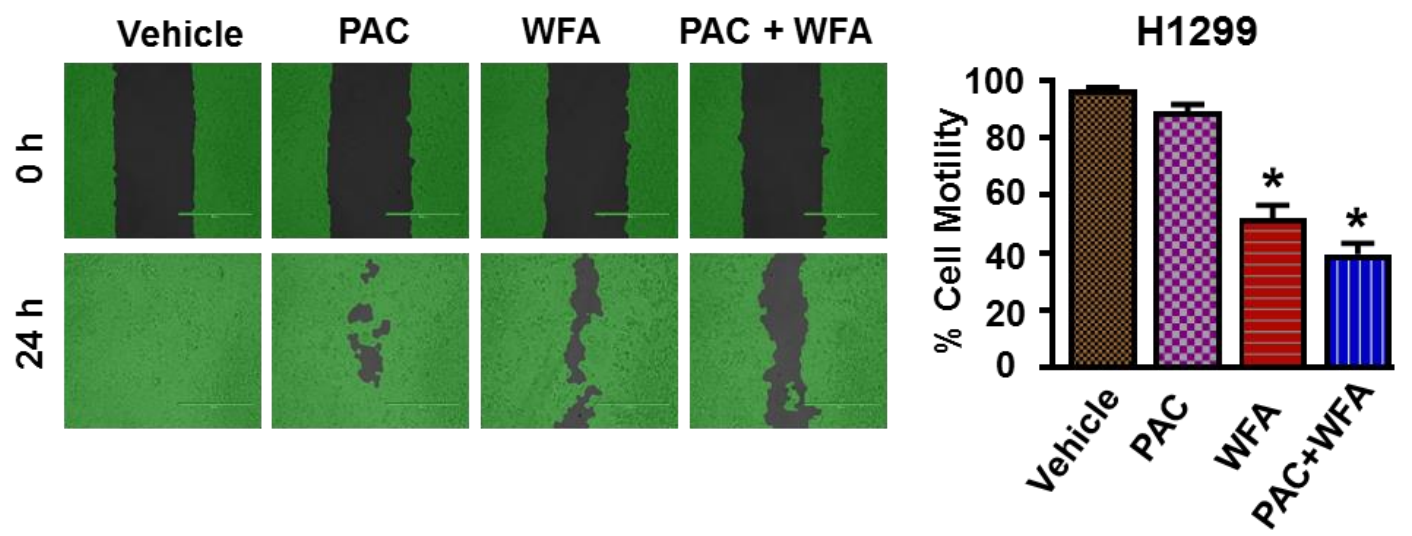

b
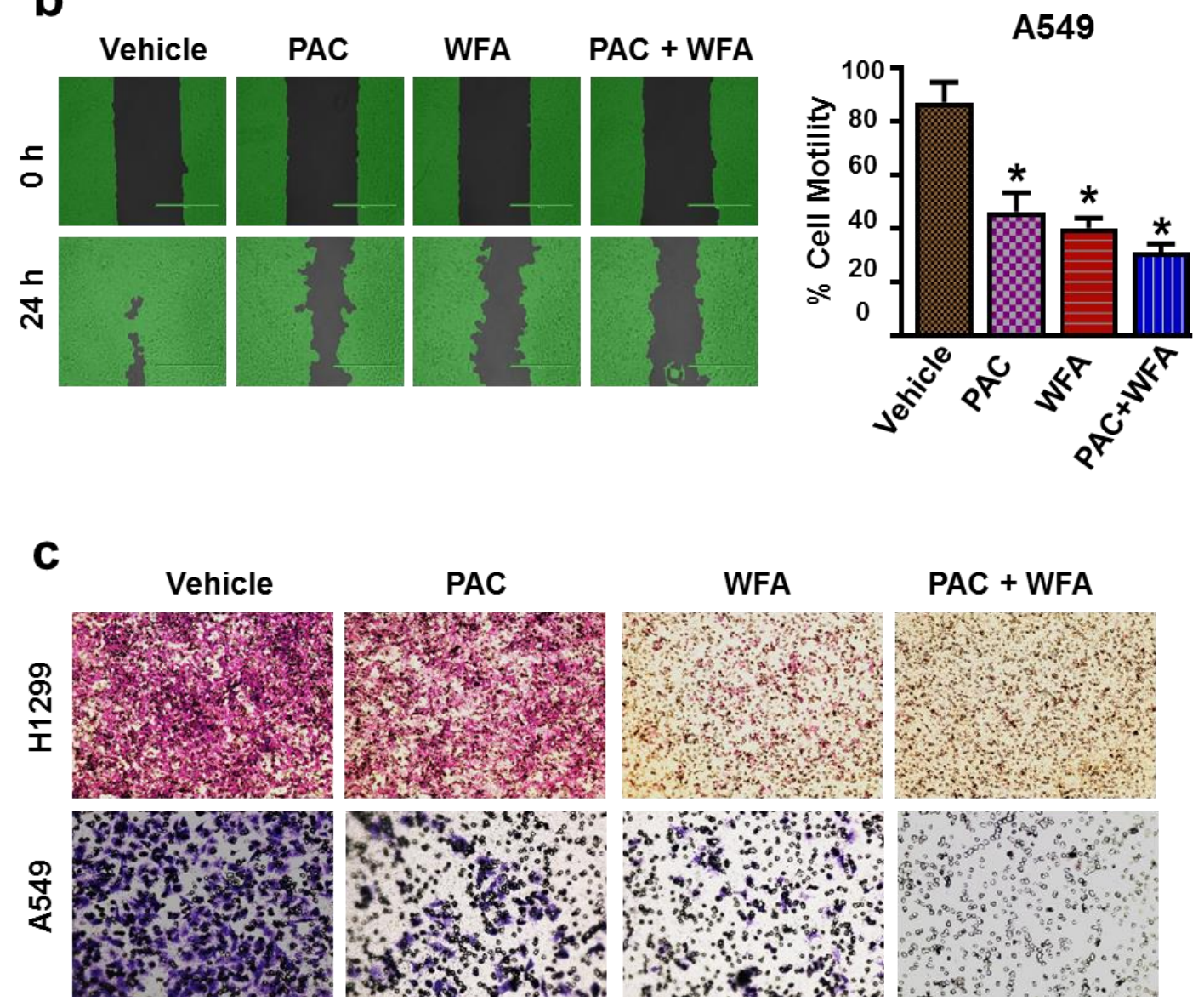

PAC + WFA

Fig 2.6. PAC and WFA inhibited NSCLC cell motility and migration. H1299 (a) and A549 (b) cells were incubated with PAC $(5 \mathrm{nM})$ and WFA $(0.2 \mu \mathrm{M})$ alone, and in combination. Cell motility was assessed by wound healing assay over $24 \mathrm{~h}$. Pictures of wound areas were taken using a light microscope and analysis by Wimasis software. (c) Invasion assay depicting the synergistic inhibition of cellular trans-well migration in $\mathrm{H} 1299$ and A549 cells. Data are mean $\pm \mathrm{SD}\left({ }^{*} \mathrm{p}<0.05\right)$. 


\section{WFA targets PAC resistant TRA549 NSCLC cells in vitro and in vivo.}

Previously published studies have reported that PAC treatment is associated with the induction and development of chemoresistance in NSCLC cells [72-74]. For example, Davi et al [75] have shown that PAC-resistance was developed in human NSCLC cell lines by culturing these cells for $>4$ months in the presence of increasing concentrations of PAC or cis-Pt. In the present study, we used PAC-resistant cells (TR-A549 cells) that were developed as described previously (Fig. 2.7a) [69] and kindly provided as a gift by Dr. Bruce Zetter (Harvard Medical School). Briefly, these TR-A549 cells were developed by starting PAC treatments $1 / 2$ PAC $\mathrm{IC}_{50}$ concentrations for $2 \mathrm{~d}$ followed by culturing the surviving cells in drug-free media for 2 wks [70]. In subsequent cycles, the last PAC concentration of the previous cycle was increased 2-fold until the development of chemoresistance. The taxol-sensitive parental (TS-A549 cells) and taxol-resistant variants (TR-A549 cells) were characterized for their drug response to PAC until we observed a 10fold change in $\mathrm{IC}_{50}$. In our studies, (Fig. 2.7b) we evaluated the sensitivity of both the drugsensitive and drug-resistant cells to PAC. Using the MTT and colony formation assays, we found that the PAC-resistant cells (TR-A549 cells) displayed cross-resistance between PAC and cis-Pt but remained sensitive to WFA (Fig. 2.7c-d). Consistent with previous studies [75], we detected increased levels of mRNA and protein expression of MDR1 and PDL-1 in the drug-resistant NSCLC cells (Fig. 2.7 e-f).

To evaluate the response of PAC-resistant TR-A549 cells to WFA, we explored the antitumor effects of WFA on the growth and proliferation of TR-A549 cells both in vitro and in vivo (Fig. 2.8). First, using in vitro assays, we demonstrated that both the parental cells (TS A549) and drug-resistant variants (TR-A549) remained sensitive to WFA 
in vitro (Fig 2.8a). To determine the effects of WFA on PAC-resistant cells in vivo, we established subcutaneous xenografts of PAC resistant cells in mice. WFA $(10 \mathrm{mg} / \mathrm{kg})$ was administered intraperitoneally in 3 doses per week in athymic nude mice xenografted with $2 \times 10^{6}$ TR-A549 cells/mouse (Fig. 2.8b). In agreement with in vitro data, mice treated with PAC (10 mg/kg divided into 3 doses a week) did not show any difference in tumor volume compared to those treated with vehicle. However, WFA significantly and time-dependently decreased the average tumor volumes of TRA549 xenografts when compared to vehicle and PAC groups. Mechanistically, WFA inhibited the mRNA and protein expression of MDR1 in TR A549 cells (Fig. 2.8c); however, modulation of PD-L1 with WFA remains to be determined. Therefore, our data presented here show that in addition to synergizing the effects of PAC, WFA targets both drug-sensitive and drug-resistant NSCLC cells. As such, the ability of WFA to synergize the anticancer efficacy of PAC and cis-Pt in drug-sensitive NSCLC cells, and target both drug-sensitive and drug-resistant NSCLC cells provides a strong rationale for the development of a combination of PAC and WFA for the treatment of NSCLC. 


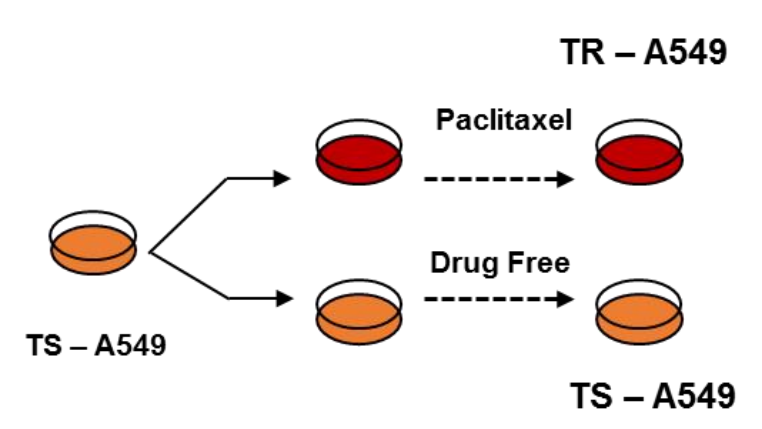

C

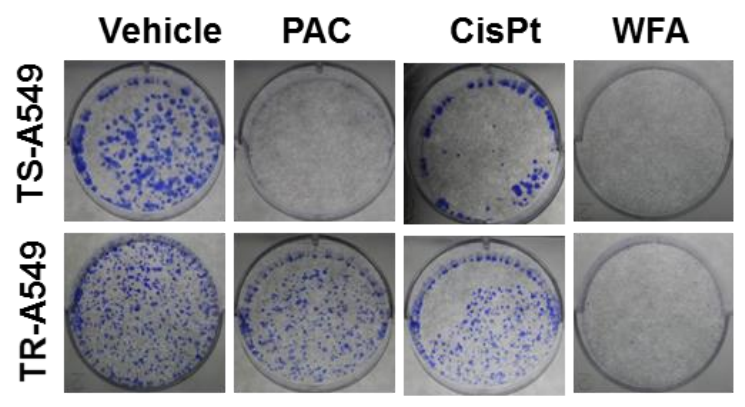

e

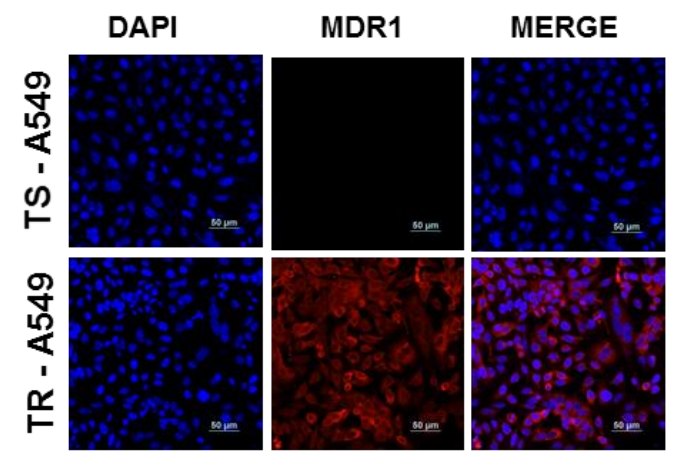

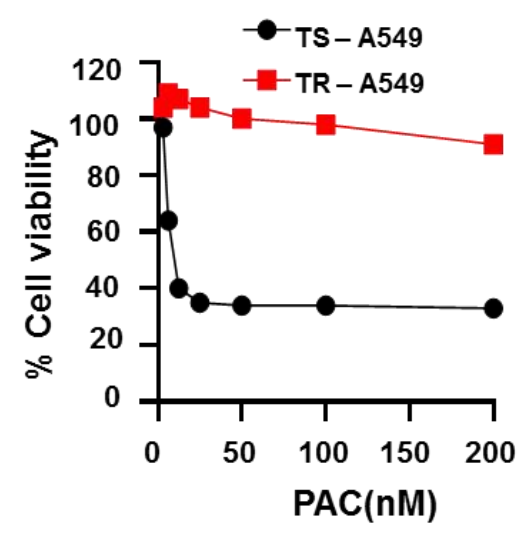

d

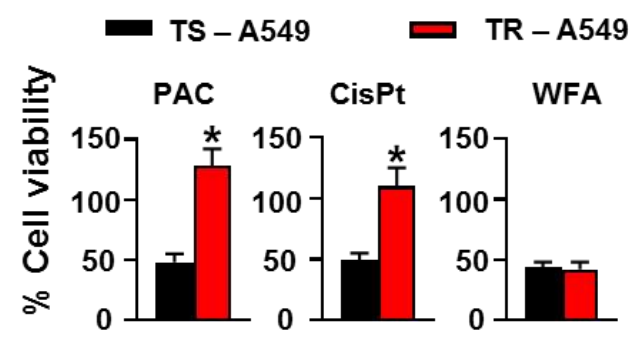

$\mathbf{f}$

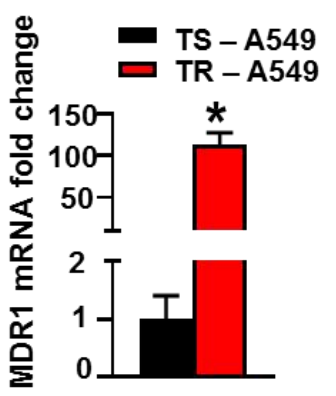

Fig 2.7. PAC treatment induced MDR1-dependent chemoresistance in NSCLC cells. (a) A549 cells were incubated in media with or without sub-cytotoxic concentrations of PAC (>6 months). (b) The cells incubated with PAC became resistant to PAC (TR-A549) while those incubated in media without PAC remained sensitive to PAC (TS-A549). (c) Colony formation assay and (d) MTT assay indicated decreased sensitivity of TR A549 cells to both PAC and CisPt but the cells remained sensitive to WFA. (e-f) Confocal imaging, (f) RT-PCR and western blot analysis indicated a significant increase in the expression of multi-resistant drug protein (MDR1). 
a

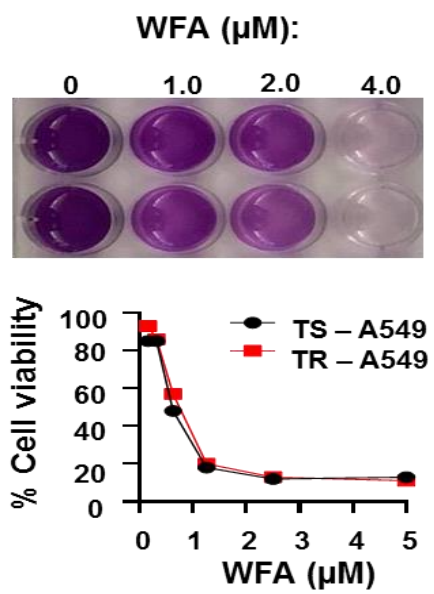

C

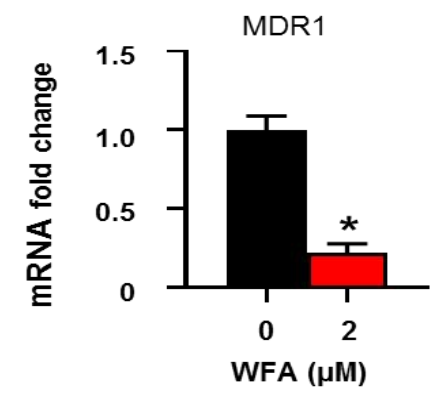

e

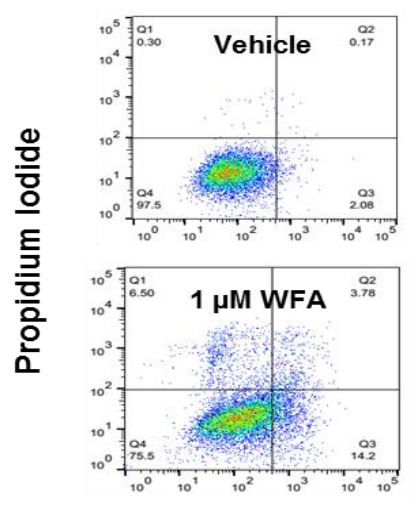

b

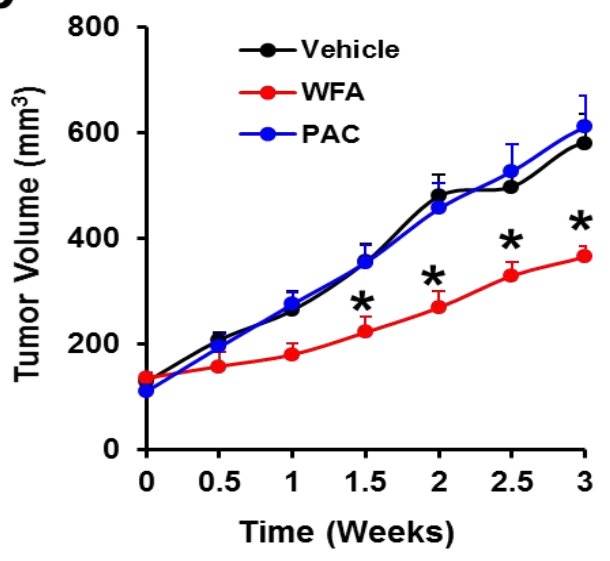

d
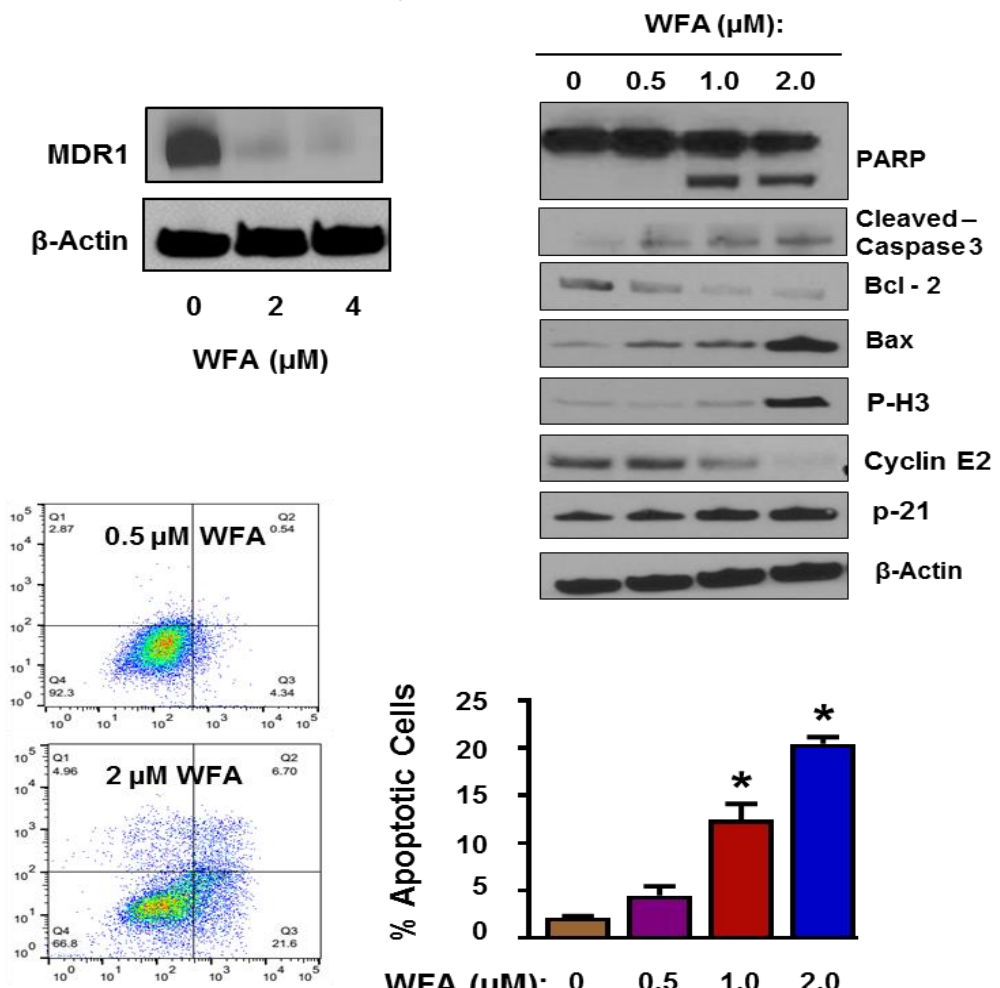

Annexin V

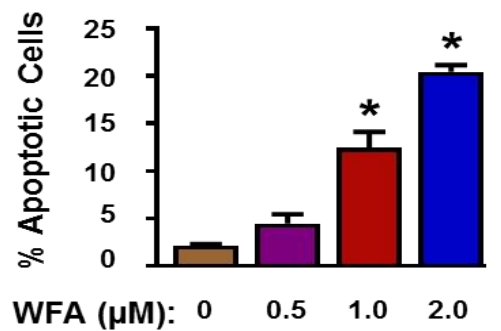

Fig 2.8. WFA alone inhibited the growth and proliferation of resistant PAC resistant NSCLC cells. (a) MTT assay depicting the in vitro inhibition of cellular proliferation of TR A549 and TS A549 (b) WFA significantly inhibited the growth of TR A549 (2.5 x10 cells/mouse) tumor xenografts in athymic nude mice. (c) RT PCR and western blot analysis indicated dose-dependent inhibition of the expression MDR1 in TR A549 after incubation with WFA for $48 \mathrm{~h}$. (d-e) AnnexinV/PI assay indicated dose-dependent induction of apoptosis in TR A549 cells after incubation with WFA $(0-2 \mu \mathrm{M})$ for $24 \mathrm{~h}$. (f) Western blot analysis confirmed the induction of apoptosis as indicated by the increased cleavage of PARP and caspase 3, increased expression of Bax, p21, phosphor-H3 while inhibiting the expression of Bc12 and cyclin E2. 


\section{DISCUSSION}

Today, taxane-platinum chemotherapeutic drug combinations remain the standard of care as first-line regimens for all sub-types of advanced NSCLC with no targetable driver genetic mutations. Cisplatin (cis-Pt) alongside paclitaxel (PAC) although highly toxic, is the most widely used and most efficacious two-drug combination for the treatment

of advanced NSCLC $[13,16,48]$. However, due to drug resistance, tumor relapse, and metastasis, the response rate has plateaued at 20 - 30\% and the survival efficacy is currently about 10-14 months [48, 49]. Moreover, despite advancements in molecularly targeted drugs and immunotherapies, chemoresistance and metastasis remain almost universal in NSCLC [46]. In addition, the currently used chemotherapeutic drugs are highly toxic, sometimes causing severe, irreversible and life-threatening toxicities in patients. Thus, the systemic treatment of advanced NSCLC remains a major therapeutic challenge, and there is a significant unmet clinical need to develop safe and efficacious alternative therapies for advanced NSCLC.

In the present study, we investigated the efficacy of various combinations of WFA and PAC synergistic against the growth and proliferation of human NSCLC cells. WFA, a plant-derived steroidal-lactone is the most prominent member of a large group of naturally occurring 28-carbon compounds called withanolides. Previously, multiple preclinical studies have extensively examined the anticancer effects of WFA on different cancer types [22]. In the current study, we demostrate that WFA is highly active against the proliferation of the human NSCLC cell lines, H1299 and A549. Mechanistically, at cytotoxic concentrations, WFA increased the generation of ROS, an indicator that WFA induces cells 
to undergo oxidative stress, and subsequently apoptosis. The generation of ROS was comfirmed by using a $\mathrm{H} 2 \mathrm{O} 2$ as a positive control and was inhibited in the presence of the ROS quencher, NAC. Interestingly, whereas we observed that ROS production was related to the cytotoxicity of WFA, only thiol containing ROS quenchers such as NAC and DTT inhibited the antitumor effects of WFA. Understandably, this phenomenon indicated that the generation of ROS was secondary to the cytotoxic antitumor activities of WFA. Therefore, although not fully investigated in present study, it appears that the molecular mechanism of action of WFA involve thiol binding as one of the more critical steps.

To establish the in vitro potency and efficacy, we have demonstrated that WFA as a single agent was active against both H1299 and A549 cells. However, since it is highly unlikely that NSCLC would be treated with monotherapy, the more attractive approach was to investigate the synergistic combination of WFA with standard chemotherapeutic drugs $[41,43,76]$. Along these line, we explored the potential for WFA to synergize the effects of PAC and/or cis-Pt against the proliferation of human NSCLC cells. This strategy was particularly exciting for the case of NSCLC since the clinical efficacy with the current treatments seems to have reached a maximum with disappointingly low survival outcomes [43]. Based on the data presented, as individual drugs, PAC, a tubulin inhibitor showed the greatest potency (lowest $\mathrm{IC}_{50}$ ) against both H1299 and A549 human NSCLC cell lines. These findings are supported by several previously published studies $[59,60]$ and strongly suggest that PAC remains the most important component of the PAC+cis-Pt combination in NSCLC treatment. As such, we took PAC to represent the standard of care and then demonstrated that its combination with WFA resulted in much greater efficacy than when combined with cis-Pt, the standard of care. 
Chou et al [71] have developed a robust method for the analysis of the synergism for two or more drug combinations using the median-effect equations. Several studies have applied this method to investigate the synergistic effects of anticancer drugs against breast [77], lung, pancreatic [76] and ovarian [41] cancers. Similarly, we used these median-effect equations as described previously to determine whether PAC and WFA were synergistic. In our studies, we demonstrated that WFA dramatically increased the efficacy and potency of both PAC and cis-Pt in a synergistic manner $(\mathrm{CI}<1)$. However, the synergistic effects observed with PAC and WFA were much greater than those for PAC and cis-Pt or WFA and cis-Pt, respectively. Interestingly, in the synergistic combinations, the observed dramatic decreases in the $\mathrm{IC}_{50}$ values were much greater for PAC than were for WFA, indicating that the greatest contribution towards synergism was from WFA in either cell lines. These observations were strongly supported by the findings from previous studies $[41,42]$ in which WFA has been previously reported to synergize the effects of standard chemotherapeutic drugs. Therefore, based on the current findings, we hypothesize that using WFA alongside PAC in NSCLC would significantly enhance clinical efficacy while dramatically decreasing the potential for toxicity in patients. In the clinic, oncologists administer chemotherapeutic drugs following complex dosing schedules to prevent overlapping toxicities. Liu et al have shown that the synergism of anticancer drugs can be enhanced by altering the sequence, concentration, schedule and duration of drug exposure [78-80]. To this effect, we investigated whether the synergism observed between PAC and WFA could be increased by altering sequence and schedule of treatment. Our findings showed that the synergistic effects of PAC and WFA were not scheduled dependent but we demonstrated that preincubation of $\mathrm{H} 1299$ and A549 cells with WFA for $2 \mathrm{~h}$ dramatically 
enhanced the antiproliferative efficacy of the combination of PAC and WFA. Thus, it could be concluded that WFA had a chemosensitizing effect on PAC against NSCLC cells as has been repoted in previous studies conducted with other cancer cell types [22].

Chemoresistance and metastasis remain the primary causes of death in advanced NSCLC with no clinically effective approved drugs [81]. At the molecular level, intrinsic and acquired resistance mechanisms in tumor cells contribute to $>80 \%$ of treatment failure in NSCLC. Furthermore, accumulating evidence from previous studies has shown that the development of chemoresistance is simultaneously induced for multiple antitumor agents following the treatment with only one agent. For example, studies $[16,70,75]$ have shown that chronic incubation of NSCLC cells with PAC treatment induces chemoresistance to cis-Pt and other chemotherapeutic agents. At the molecular level, PAC induces cellular mechanisms such as EMT in NSCLC cells that trigger the development of chemoresistance and metastasis. In the present study, we used a previously developed PAC resistant (TRA549) cell line that expressed high levels of MDR1 indicating that chemoresistance was mediated by increased drug-efflux from cells. Also, we observed high levels of PDL-1 expression in TR-A549 cells which strongly suggested a link between the simultaneous development of drug resistance and immune evasion in NSCLC [82]. Using this TR-A549 cell line, we demonstrated that WFA was active against both drug-sensitive and drugresistant NSCLC tumor cells. Since WFA was active against this MDR-1 overexpressing TR-A549 cell line, it appears that WFA is not a substrate for MDR-1 and has the potential to inhibit MDR-1 dependent drug resistance in NSCLC. Furthermore, the TR-A549 cells displayed decreased E-cadherin expression, a molecular indicator that PAC induced an EMT phenotype in NSCLC. In a recent study [83], we have shown that WFA inhibits EMT 
in NSCLC cells, and we therefore strongly hypothesize that the use of PAC and WFA can prevent the emergence of drug resistance and metastasis in NSCLC. Together, these novel findings strongly support our rationale for combining PAC with WFA, and further suggest that this strategy has an additional benefit of the ability to prevent the emergence or target drug resistance and metastasis in NSCLC.

In conclusion, we have investigated and demonstrated the therapeutic potential of WFA alone and in combination with PAC against human NSCLC cells. Using in vitro antiproliferative assays, we have shown that PAC and WFA are highly synergistic against H1299 (large cell carcinoma) and A549 (adenocarcinoma) cells. Moreover, the PAC and WFA combinations were more efficacious than PAC and cis-Pt or WFA and cis-Pt, respectively, against both $\mathrm{H} 1299$ and A549 human NSCLC cells. In addition, WFA was active against the proliferation of the drug-resistant (TR-A549) cells and decreased the expression of MDR-1 in these cells. Together these findings provide a strong rationale for further development of the PAC and WFA combination to advance a new therapeutic alternative for the treatment of advanced NSCLC. 


\section{CHAPTER THREE}

\section{WITHAFERIN A INHIBITS EPITHELIAL TO MESENCHYMAL TRANSITION IN NON-SMALL CELL LUNG CANCER CELLS}

\section{INTRODUCTION}

Lung cancer is the leading cause of cancer-related deaths worldwide [10] and in the United States [44]. Non-small cell lung cancer (NSCLC) which accounts for about 85-90\% of all lung cancer cases has an overall five-year survival rate of 15-17\% [84, 85]. Despite recent advancements in early detection methods [81,86], surgical techniques, targeted and immunotherapies [87], the overall survival from NSCLC has only minimally improved. This poor prognosis is explained in part because about 50-70\% of all NSCLC patients are diagnosed when the disease is at an advanced stage and are not curable regardless of treatment approach [81]. Furthermore, the rate of tumor recurrence among NSCLC patients who undergo surgical resection is about $30-70 \%$, most of whom eventually succumb to metastasis $[15,45]$.

Tumor cell migration, invasion, and metastasis are the primary causes of treatment failure and death among NSCLC patients [84, 88]. Unlike cellular proliferation, the therapeutic targeting of metastasis is difficult with no clinically-effective drugs. Metastatic processes are complex, and the underlying mechanisms are still poorly understood. because they utilize an interplay of cell adhesion, motility, and survival pathways [89]. 
Recently, several clinical and preclinical studies indicate that epithelial-to-mesenchymal transition (EMT) plays is a crucial role in the metastatic process of NSCLC cells $[90,91]$. EMT is a complex but dynamic biochemical process of cellular reprogramming where epithelial cells undergo extensive molecular and morphological changes to acquire a mesenchymal phenotype. Normally, EMT critical during embryogenesis, angiogenesis and wound healing but tumor cells invoke EMT to increase their motility, migratory, and invasive capacities [92, 93]. The importance of EMT in metastasis has prompted the evaluation of small molecule inhibitors of EMT as potential drugs against metastasis in NSCLC [89].

Withaferin A (WFA) is a biologically active steroidal-lactone that was first isolated from the extracts of the Indian Ayurvedic medicinal plant, Withania somnifera [24]. Previous studies have demonstrated multiple pharmacological effects of WFA including antitumor, anti-inflammatory, pro-apoptotic, immunomodulatory, hepatoprotective, anabolic, antiangiogenic, and anti-fibrotic activities. Despite these multiple effects, WFA has attracted significant preclinical testing mainly for its antitumor activities [22]. Indeed, WFA has been shown to be cytotoxic against lung [40, 62-64], cervical [66], prostate [39, 94], breast [31, 35, 37], ovarian [41-43], colon [95], B-cell lymphoma [68], and pancreatic [76] cancers in the preclinical setting. More importantly, in addition to cytotoxicity, WFA displays remarkable anti-invasive and anti-metastatic activities [22,31,96]. Although the molecular mechanisms underlying the activity of WFA are not fully understood, its antimetastatic effects have been attributed in part to its inhibition of EMT in tumor cells.

Previously, anti-EMT properties of WFA have been demonstrated on breast, ovarian, and mammalian epithelial cells [22]. In the present study, we examined the effects of WFA 
on migration, invasion, and EMT induction in A549 and H1299 cells. Here, we show that the cytokines TGF $\beta 1$ and TNF $\alpha$ cooperate to induce EMT, increase migration and invasion of these tumor cells. Importantly, we demonstrated that sub-cytotoxic doses of WFA suppressed TGF $\beta / \mathrm{TNF} \alpha$-induced EMT, cell adhesion, migration, invasion, and motility. Mechanistically, WFA inhibited TGF $\beta 1$-dependent Smad2/3-signaling and TNF $\alpha$ dependent NFKB signaling. Together, this data provides additional evidence on role WFA in regulating EMT in tumor cells and further demonstrates the therapeutic potential of WFA for the treatment of metastatic NSCLC. 


\section{MATERIALS AND METHODS}

\section{Reagents, chemicals, and supplies.}

WFA ( $>96 \%$ pure) was generously provided as a gift sample by 3P Biotechnologies Inc., (Louisville, KY, USA). A stock solution of WFA (5 mM) was prepared in dimethyl sulfoxide (DMSO) and stored in aliquots at $-20^{\circ} \mathrm{C}$ until use. MTT (3-[4,5-dimethylthiazol2-yl]-2,5-diphenyltetrazolium bromide) was purchased from Alfa Aesar (Ward Hill, MA, USA). RIPA cell lysis buffer, BCA protein assay kit, PVDF membranes, phosphatebuffered- saline (PBS), ECL chemiluminescence reagent, and Bolt 4-12\% Bis-Tris phosphate gels were purchased from ThermoFisher (Rockford, IL, USA). Dulbecco's modified Eagle's medium (DMEM), penicillin and streptomycin were purchased from Life Technologies (Gibco, Grand Islands, NY, USA). Fetal bovine serum (FBS) was purchased from VWR, Seradigm Life science (Radnor, PA, USA). The human recombinant TGF $\beta-1$ (\#8915) and TNFa (\#8902) were purchased from Cell Signaling Technology (Danvers, MA, USA) and prepared as $50 \mu \mathrm{g} / \mathrm{mL}$ stock solutions in sterile $20 \mathrm{mM}$ citrate, $\mathrm{pH} 3.0$ and sterile PBS, respectively. The stock solution was stored in aliquots at $-80^{\circ} \mathrm{C}$ and diluted in serum-free DMEM media before use.

\section{Antibodies.}

Primary antibodies against Smad2/3, phospho-Smad2/3, Smad 2, Smad3, phospho-Smad2, phospho-Smad3, Smad4, Smad7, Snail, Slug, E-cadherin, ZEB1, N-cadherin, Vimentin, $\mathrm{NF} \kappa \mathrm{B}, \mathrm{pNF} \kappa \mathrm{B}, \mathrm{I} \kappa \mathrm{B}$, phospho-I $\mathrm{B}, \mathrm{IKK} \alpha, \mathrm{IKK} \beta$, phospho-IKK $\alpha / \beta$, and were purchased from Cell Signaling Technology (Danvers, MA). The primary antibodies against $\beta$-actin 
were purchased from Sigma Chemical Co. (St. Louis, MO). A detailed list of all antibodies used is presented in Supplementary Table 1.

\section{Cell culture conditions.}

The human NSCLC cell lines, A549, and H1299 were maintained in DMEM media supplemented with $10 \%(v / v)$ fetal bovine serum (FBS) and $1 \%$ antibiotics $(100 \mathrm{U} / \mathrm{mL}$ penicillin and $100 \mu \mathrm{g} / \mathrm{mL}$ streptomycin), at $37^{\circ} \mathrm{C}$ with $5 \% \mathrm{CO}_{2}$ in a humidified incubator. Culture media was replaced every $48 \mathrm{~h}$ and the cells were passaged at $\sim 80 \%$ confluence, for less than 20 cycles.

\section{Cell viability assay.}

Cell viability was determined by MTT assay as described previously. Briefly, cells were seeded in 96-well plates $\left(3 \times 10^{3}\right.$ cells/well) and preincubated for $24 \mathrm{~h}$ before treatments. DMEM was replaced with fresh media containing various concentrations $[0-5 \mu \mathrm{M}]$ of WFA and incubated for an additional 24,48 , and $72 \mathrm{~h}$. The cells were then incubated with the $0.5 \mathrm{mg} / \mathrm{mL}$ MTT reagent for $3 \mathrm{~h}$ and formazan crystals formed were solubilized using $200 \mu \mathrm{L}$ of DMSO. The absorbance of the resulting solution was measured at $570 \mathrm{~nm}$. Data were expressed as mean $\pm \mathrm{SD}$ of four or more separate experiments.

\section{Cell adhesion assay.}

Cells were serum starved for $12 \mathrm{~h}$ and then incubated with WFA in the presence or absence of TGF $\beta 1$ or TNF $\alpha$ in serum-free media for $1 \mathrm{~h}$. The cells were then seeded in Matrigel (50 $\mu \mathrm{g} / \mathrm{mL})$ pre-coated 96 -well plates $\left(5 \times 10^{4}\right.$ cells/ well) and incubated for $2 \mathrm{~h}$ to allow attachment. Non-adherent cells were washed away using pre-warmed sterile PBS, and then

fresh DMEM media (10\% FBS) was added to each well. Cultures were incubated for an additional $4 \mathrm{~h}$, and then cell viability was measured using MTT assay. 


\section{Colony formation assay.}

Cells were incubated with or without WFA for $24 \mathrm{~h}$, trypsinized and then seeded in 6well plates at 200 cells/well density. Fresh DMEM media was added and changed every $48 \mathrm{~h}$ for a total of $7 \mathrm{~d}$. The colonies formed were fixed using methanol/acetic acid solution (3:1), and stained with $0.2 \%$ crystal violet. The number of colonies formed was recorded using a high-resolution digital camera and counted using a microscope in five random fields.

\section{Wound healing assay.}

The wound-healing assay was performed using the 2-well inserts from ibidi ${ }^{\circledR}$ (Munich, Germany). Briefly, cells ( $1 \times 10^{4}$ cells/well) were seeded into the 2 -well inserts and incubated for $24 \mathrm{~h}$ to allow cell attachment. The cell-free gaps (wounds) were created by removing the culture inserts. Floating cells and cellular debris were washed off using sterile PBS, and media was replaced with fresh media containing WFA in the presence or absence of TGF $\beta 1$ and TNF $\alpha$. The distance migrated by cells in each cell-free gap was monitored by light microscopy at 0,12 , and $24 \mathrm{~h}$. At each time point, 3 random microphotographs of the wound area were taken. The percent migration was quantified using Wimasis Image Analysis software (WimScratch, Cordoba, Spain).

\section{Transwell migration and invasion assays.}

Migration and invasion assays were performed using uncoated and Matrigel-coated $8 \mu \mathrm{m}$

pore transwell chambers (BD Bioscience, San Jose, CA), respectively. Cells $\left(4 \times 10^{4}\right.$ cells/well) were suspended in serum-free DMEM media and seeded into the upper compartments of transwells. DMEM media containing 10\% FBS was added to the lower 
chamber as a chemoattractant to cause cell migration from the upper chamber to the lower chamber of the transwells. Cell migration was allowed to occur for $24 \mathrm{~h}$, and the migrated cells were fixed using $4 \%$ paraformaldehyde solution. Cells were permeabilized using $100 \%$ methanol and then stained using $0.2 \%$ toluidine blue. Migrated cells in five random fields were examined using a microscope and microphotographs taken using a highresolution digital camera.

\section{Western blot analysis.}

Following incubation with indicated treatments, cells were harvested by trypsinization and washed twice with ice-cold PBS. Whole-cell protein lysates were prepared by suspending cell pellets in RIPA cell lysis buffer at $4^{\circ} \mathrm{C}$. Protein concentration for the cellular lysate was determined by BCA method and protein samples $(20 \mu \mathrm{g})$ were resolved by SDS-PAGE

and then transferred to PVDF membranes. The expression of specific proteins was determined by probing the PVDF membranes with specific primary monoclonal antibodies, followed by the respective HRP-conjugated secondary antibodies. The levels of each protein were determined by visualizing protein bands on blots using ECL detection reagents (Thermo Scientific, Rockford, IL). The relative density of each blot was quantified using the ImageJ software (NIH, Bethesda, MD). $\beta$-actin (Sigma-Aldrich, St. Louis, MO) was used as protein loading controls.

\section{Electrophoretic Mobility Shift Assay (EMSA).}

To determine the effect of WFA on the binding of NFKB to nucleic acids, the electrophoretic mobility shift assay (EMSA) was performed. Briefly, cells were incubated with or without WFA for $24 \mathrm{~h}$, and then was NFkB stimulated using $5 \mathrm{ng} / \mathrm{mL}$ TNF $\alpha$ for 30 
min. Cytosolic and nuclear cell extracts were prepared using standard protocols. A total of $30 \mu \mathrm{g}$ nuclear protein was incubated with ${ }^{32} \mathrm{P}$ end-labeled double-stranded NFkB, and the DNA-protein complex formed were separated from free oligonucleotide on $6 \%$ native polyacrylamide gels. The radioactive bands were visualized by a Packard InstantImager.

\section{Immunofluorescence staining.}

Cells were seeded in 8-well chamber slides and cultured up to $60-70 \%$ confluency. After $24 \mathrm{~h}$ serum starvation, cells were incubated with $\mathrm{TGF} \beta 1$ or $\mathrm{TNF} \alpha$ in the presence or absence of WFA for $24 \mathrm{~h}$. Then, cells were fixed using $4 \%$ paraformaldehyde and permeabilized using $0.25 \%$ Triton $\mathrm{X}-100$ in PBS. The fixed cells were blocked using $1 \%$ BSA for $1 \mathrm{~h}$ at $23^{\circ} \mathrm{C}$ and then probed with specific primary antibodies at $4{ }^{\circ} \mathrm{C}$ overnight. The primary antibodies were then probed with fluorescent-tagged secondary monoclonal antibodies diluted in $1 \% \mathrm{BSA}$ in PBS for $2 \mathrm{~h}$ at $23^{\circ} \mathrm{C}$. F-actin was stained using green or red fluorescent phalloidin while the nuclei were stained blue using DAPI.

\section{qRT-PCR analysis.}

Total RNA was extracted from cells using Trizol reagent (Invitrogen, Carlsbad, CA) as per the manufacturer's instructions. The relative expression of E-cadherin, vimentin, Snail, and fibronectin mRNA levels were determined by qRT-PCR and normalized to the expression of GAPDH. qRT-PCR was performed using Power SYBR Green RNA to CT 1-Step Kit. The primers used in the qRT-PCR assay are listed in table 1 in the supplementary information. Relative mRNA levels were determined by calculating the fold change for each sample using the $2^{-\Delta \Delta C t}$ relative quantification method. 


\section{Statistical analysis.}

Statistical analysis was performed using Graph Pad Prism 7.0 software. Data were presented as means \pm SD of at least 3 separate experiments. First, One-way analysis of variance (ANOVA) was used to determine variations among experimental and control groups. Comparisons of individual experimental and control group means were done using the students' t-test, and p-values $<0.05$ were considered statistically significant. 


\section{RESULTS}

WFA inhibits the growth and proliferation of A549 and H1299 cells.

First, we determined the cytotoxic effects of WFA against the two human NSCLC cell lines H1299 and A549, which are known to be highly and moderately metastatic, respectively. Incubation of cells with WFA caused dose- and time-dependent cytotoxicity to both A549 and H1299 cells. Phase-contrast images (Fig. 3.1b) were obtained after $48 \mathrm{~h}$ of cell culture in the presence of WFA. The images revealed significant dose-dependent changes in cellular morphology of both A549 and H1299 cells, that were indicative of decreased cell viability and/or increased cell death. To quantitatively determine the cytotoxic effects of WFA on A549 and H1299 cells, we used the MTT assay to measure cell viability. By considering the vehicle-treated cells as $100 \%$ viable, our data (Fig. 3.1c) shows that WFA dose-dependently decreased the viability of both A549 and H1299 cells. The anti-proliferative effects of WFA on NSCLC cells were both time- and concentrationdependent (Fig. 3.1d-e).

Next, to validate the MTT data, we assessed the effects of WFA on the replicative ability of A549 and H1299 cells by conducting the colony formation assay. Representative images (Fig. 3.1f) of A549 and H1299 colonies stained using 0.5\% crystal violet in methanol. In support of our MTT assay data, WFA significantly inhibited the ability of cells to form colonies. Together, this data demonstrates the remarkable cytotoxic effects of WFA against A549 and H1299 human NSCLC cells in vitro. Overall, we found the highly invasive H1299 cells to be more sensitive to WFA than the moderately invasive A549 cells. 
b
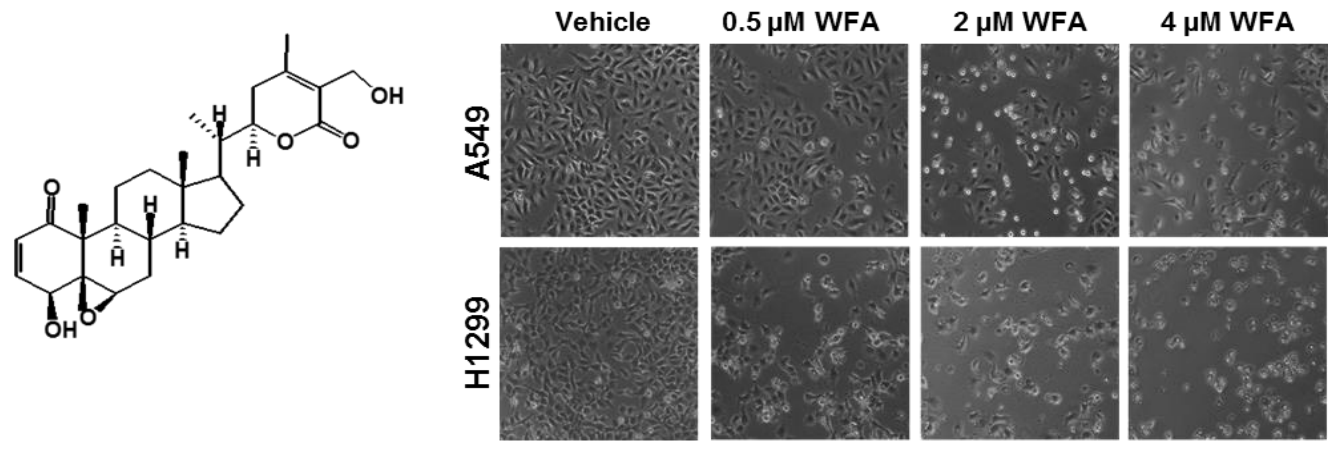

C

d
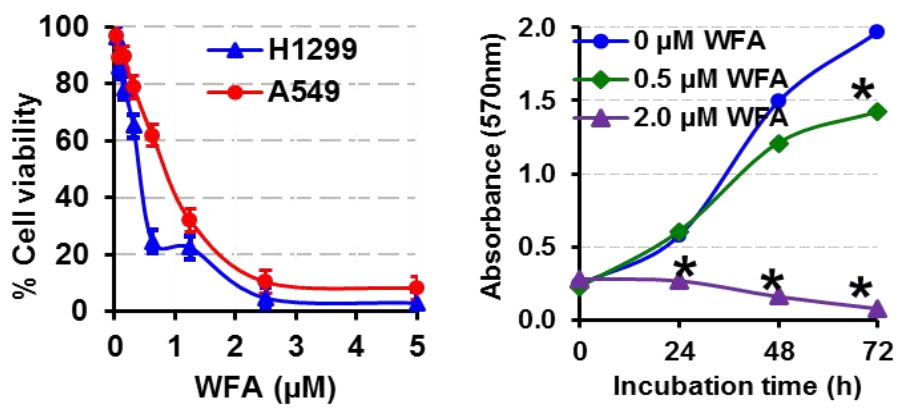

e

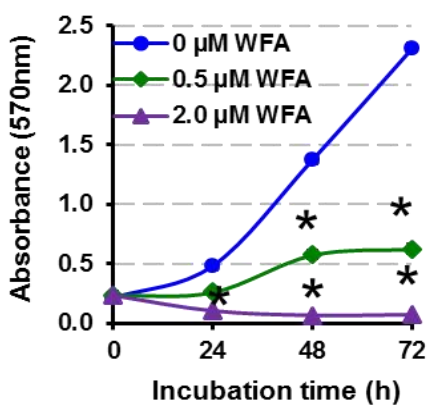

$\mathbf{f}$
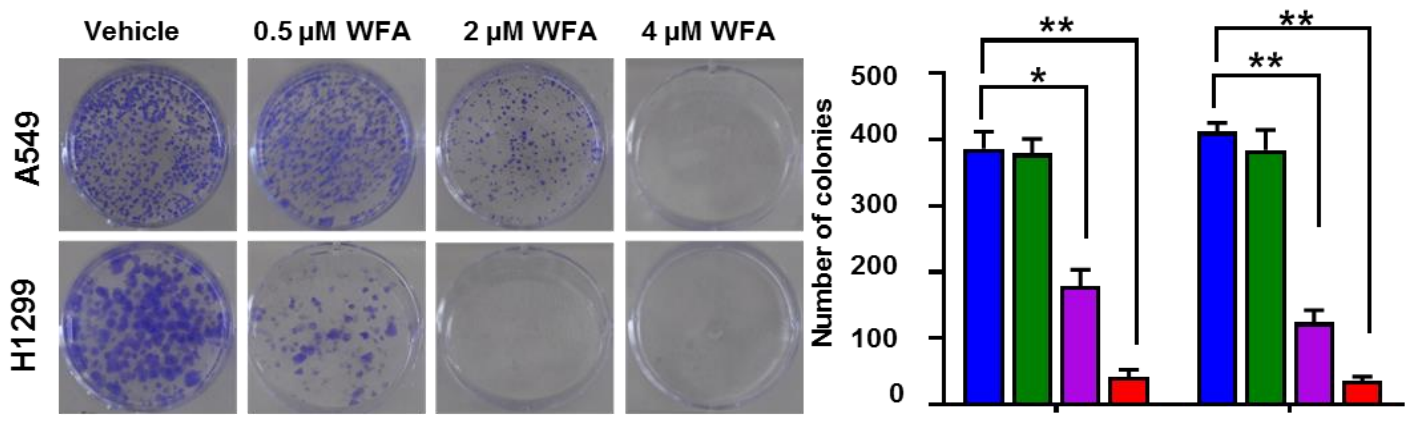

WFA $(\mu \mathrm{M}): \begin{array}{llllllll}0 & 0.5 & 2 & 4 & 0 & 0.5 & 2 & 4\end{array}$

Fig 3.1. WFA suppressed the growth and proliferation of A549 and H1299 cells. (a) Chemical structure of WFA. (b) Phase-contrast images (4X magnification) showing the cellular morphology following incubation with WFA for $48 \mathrm{~h}$. (c) Dose-dependent antiproliferative activity of WFA against A549 and H1299 cells after 72 h incubation. Time- and concentration-dependent inhibitory effects of WFA on the growth and proliferation of A549 (d) and (e) H1299 cells. (f) Representative images of colony formation assay depicting a dose-dependent inhibitory effect of WFA on A549 and H1299 cells. Cell viability was measured using MTT assay while the number of colonies formed were counted using a microscope and data are presented as mean $\pm \mathrm{SD}$ of 3 technical replicates. ${ }^{*} \mathrm{p}<0.05, * * \mathrm{p}<0.01$. 


\section{WFA inhibits cell adhesion, migration, and invasion of A549 and H1299 cells}

Increased migratory and invasive behaviors of tumor cells are known to be indicative of higher metastatic potential in NSCLC tumors. Therefore, to test whether WFA inhibited the metastatic potential of A549 and H1299 cells, we conducted the cell adhesion, migration, and invasion assays. We used the cell adhesion assay to determine the potential of A549 and H1299 to attach to an extracellular matrix in the presence of WFA. The vehicle-treated cells (Fig. 3.2a) were considered to have $100 \%$ adhesion to Matrigel, while WFA treated cells displayed dose-dependent in cell adhesion for both A549 and H1299 cells. Next, to examine the effects of WFA on cell motility, we performed the wound healing assay for both A549 and H1299 cells. We observed (Fig. 3.2b) that $0.5 \mu \mathrm{M}$ WFA significantly suppressed cell motility of both A549 and H1299 cells. In untreated (vehicle-treated) cells, $>95 \%$ of the wound areas were covered by cells indicating higher cell motility. However, in the presence of WFA, we observed only 20$40 \%$ motility of these cells $(* \mathrm{p}<0.05)$.

Furthermore, we also determined the effect of WFA on transwell migration and invasion using $8 \mu \mathrm{m}$-pore transwell chambers coated with or without Matrigel. The number of migrated cells were counted in five random fields under a microscope and presented as mean \pm SD. Representative microphotographs of migrated and invaded cells show that WFA significantly suppressed the migration and invasion of A549 and H1299 cells (Fig $3.2 c)$. 
a

A549
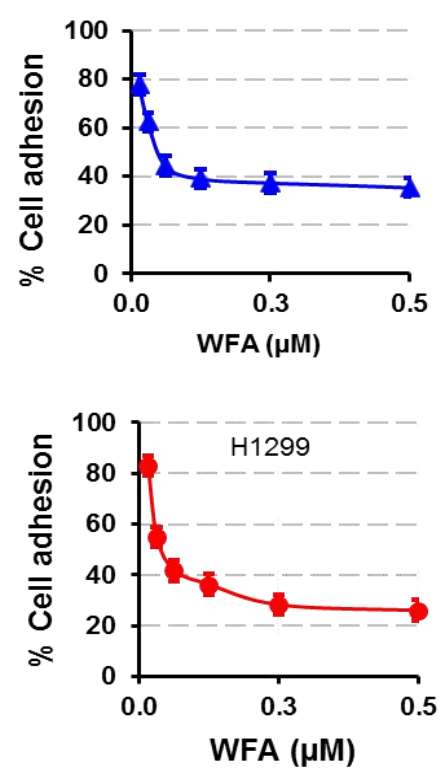

C
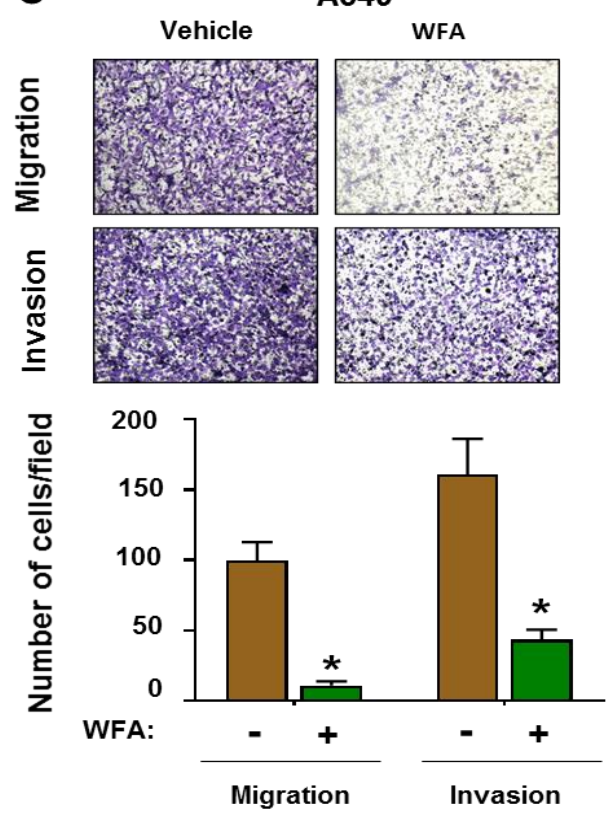

A549

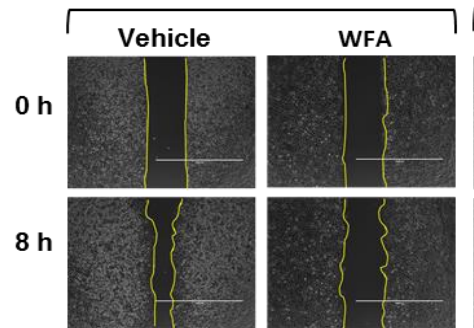

$24 \mathrm{~h}$
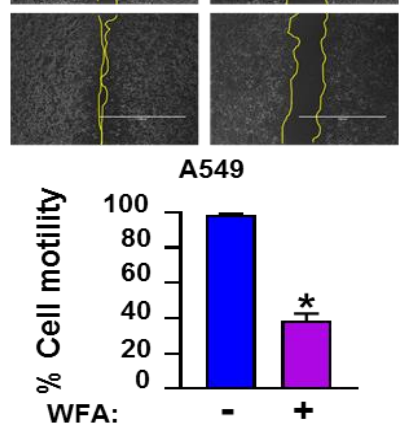

H1299
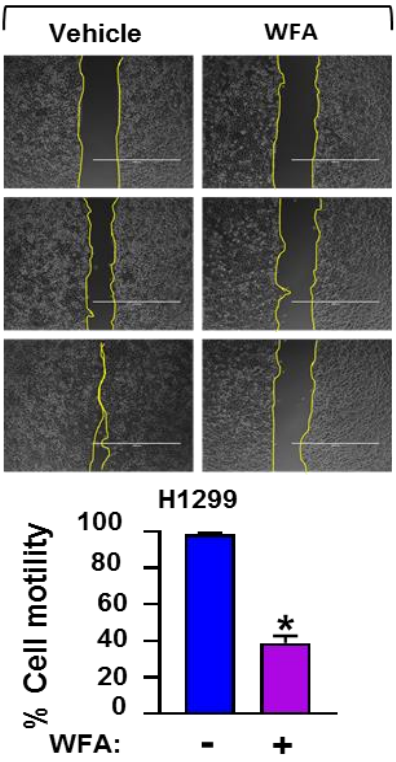

H1299

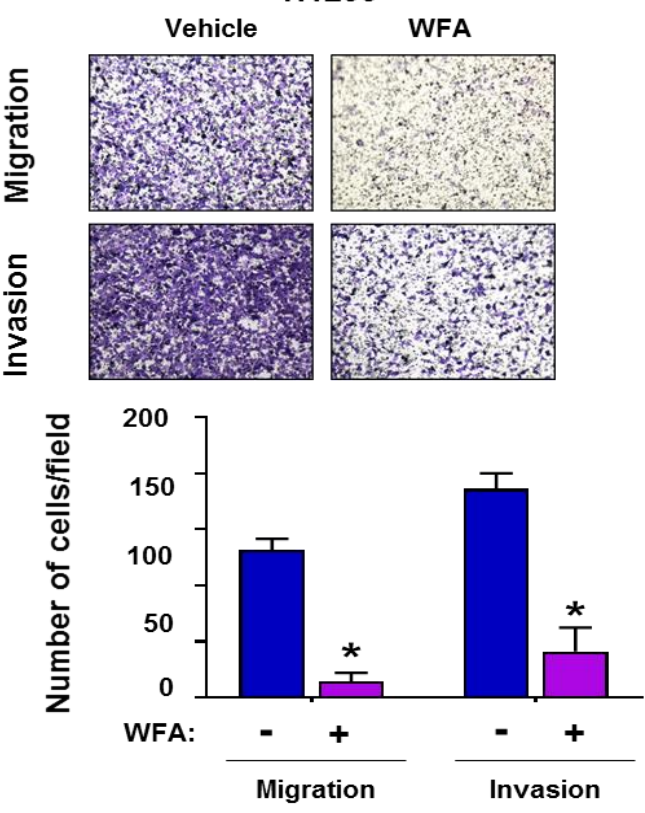

Figure 3.2. WFA inhibited cell adhesion, motility, migration, and invasion of A549 and H1299 cells. (a) Cell adhesion assay depicting the dose-dependent inhibition of the adhesion of A549 and H1299 cells. (b) Wound healing assay showing the inhibitory effect of WFA on the motility of A549 and H1299 cells. (c) Representative images showing the effect of WFA on transwell migration and invasion of A549 and H1299 cells following incubation with WFA. Data are mean $\pm \mathrm{SD},{ }^{*} \mathrm{p}<0.05$. 


\section{WFA inhibited experimental EMT induction in A549 and H1299 cells.}

The loss of the epithelial marker E-cadherin and an increase in the expression of $\mathrm{N}$-cadherin (also known as the cadherin switch) is the hallmark of EMT induction in tumor cells. Previously, TGF $\beta 1$ and TNF $\alpha$ either individually or in combination have been used to induce experimental EMT in various tumor cell types including NSCLC. In the present study, we assessed whether WFA could inhibit experimentally induced EMT in A549 and H1299 cells. First, we induced EMT in A549 and H1299 cells by culturing cells in serum-free media containing TGF $\beta 1(5 \mathrm{ng} / \mathrm{mL})$ and TNF $\alpha(25 \mathrm{ng} / \mathrm{mL})$ alone or in combination. Data presented (Fig. 3.3a) shows the western blot analysis of whole-cell lysates prepared from A549 and H1299 cells following EMT induction. In the vehicle-treated cells, the highly invasive H1299 cells did not express E-cadherin but A549 cells expressed significant levels of E-cadherin. However, incubation of A549 cells with TGF $\beta 1$ and TNF $\alpha$ either alone or in combination resulted in a significant decrease in the expression of E-cadherin in A549 cells while upregulating N-cadherin. This finding indicated that culturing A549 cells in the presence of these cytokines resulted in EMT induction. Using qRT-PCR analysis (Fig. 3.3b), it was found that the repression of E-Cadherin expression was associated with decreased levels of mRNA in A549 cells. On the other hand, the expression levels of the EMT-related proteins such as vimentin, Snail, fibronectin, and claudin-1 were all significantly increased after incubation with the cytokines. Interestingly, the expression of Snail, a known EMT transcription factor was significantly increased by TGF $\beta 1$ alone or in the combination of TGF $\beta 1$ and TNF $\alpha$ but not TNF $\alpha$. Also, only TGF $\beta 1$ significantly increased the expression of vimentin as indicated by western blot (Fig. 3.3a) and qRT-PCR (Fig.3.3b). 
a

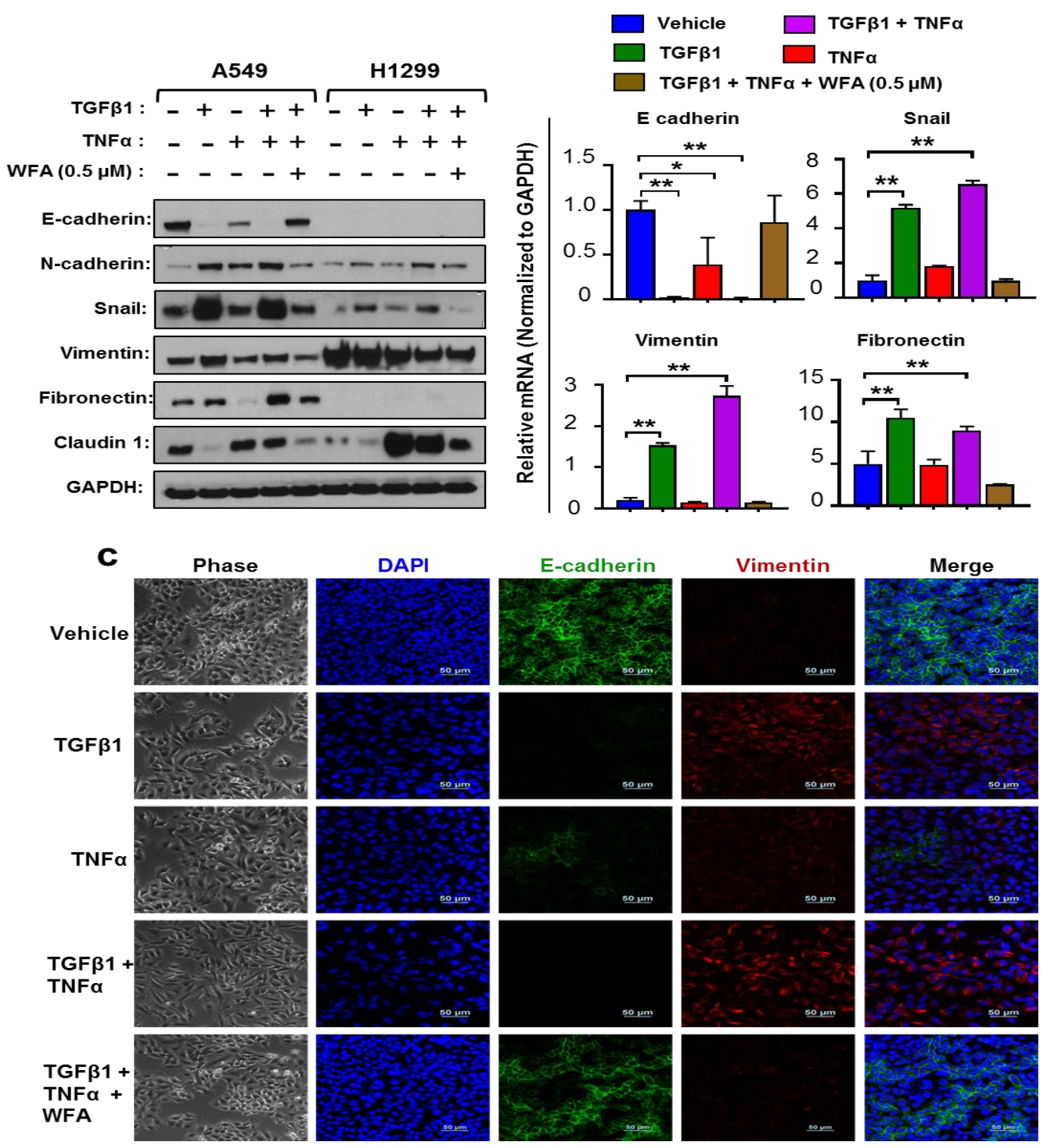

Fig 3.3. WFA inhibited experimental EMT induction in A549 and H1299 cells. Cells were left untreated or pretreated with $0.5 \mu \mathrm{M}$ WFA for $12 \mathrm{~h}$ and then stimulated with TGF $\beta 1$ or TNF $\alpha$ alone and in combination in serum free media. EMT induction was assessed by western blot analysis, qRT-PCR, and immunofluorescence staining. (a) Western blot analysis depicting the expression levels the epithelial marker E-cadherin, and the EMT-related proteins N-cadherin, Snail, vimentin, and fibronectin (b) qRT-PCR depicting the relative mRNA expression. Fold change was calculated using the $\Delta \Delta \mathrm{CT}$ method and GAPDH was used as normalizing gene. (c) Phase-contrast images (4x magnification) and confocal images showing changes in cellular morphology and the expression of E-cadherin and vimentin in A549 cells following incubation with a combination of TGF $\beta 1$ and TNF $\alpha$ in the presence or absence of $0.5 \mu \mathrm{M}$ WFA. $(* \mathrm{p}<0.05$ vs. untreated). 
When cells were pre-incubated with $0.5 \mu \mathrm{M}$ WFA prior to EMT induction, the observed repression of E-cadherin or the upregulation of vimentin, Snail, fibronectin, and claudin1 were inhibited. This inhibitory effect of WFA was confirmed using both western blot analysis and qRT-PCR analysis. To further validate these data, we performed immunofluorescence staining to determine the expression of E-cadherin and vimentin in A549 cells following EMT induction. Phase contrast and confocal images (Fig 3.3c) show the morphological changes, downregulation of E-cadherin, and the upregulation of vimentin as indicators of EMT induction in A549 cells. There was evidence of loss of cell to cell contact, and the cells became more elongated. However, in support of data obtained by western blot and qRT-PCR analysis, WFA significantly inhibited EMT induction in A549 cells. Further, we assessed the effects of WFA motility of both A549 and H1299 cells in the presence of TGF $\beta 1$ and TNF $\alpha$. As indicated (Fig. 3.4), TGF $\beta 1$ and TNF $\alpha$ significantly increased the motility which was suppressed in the presence of WFA. 

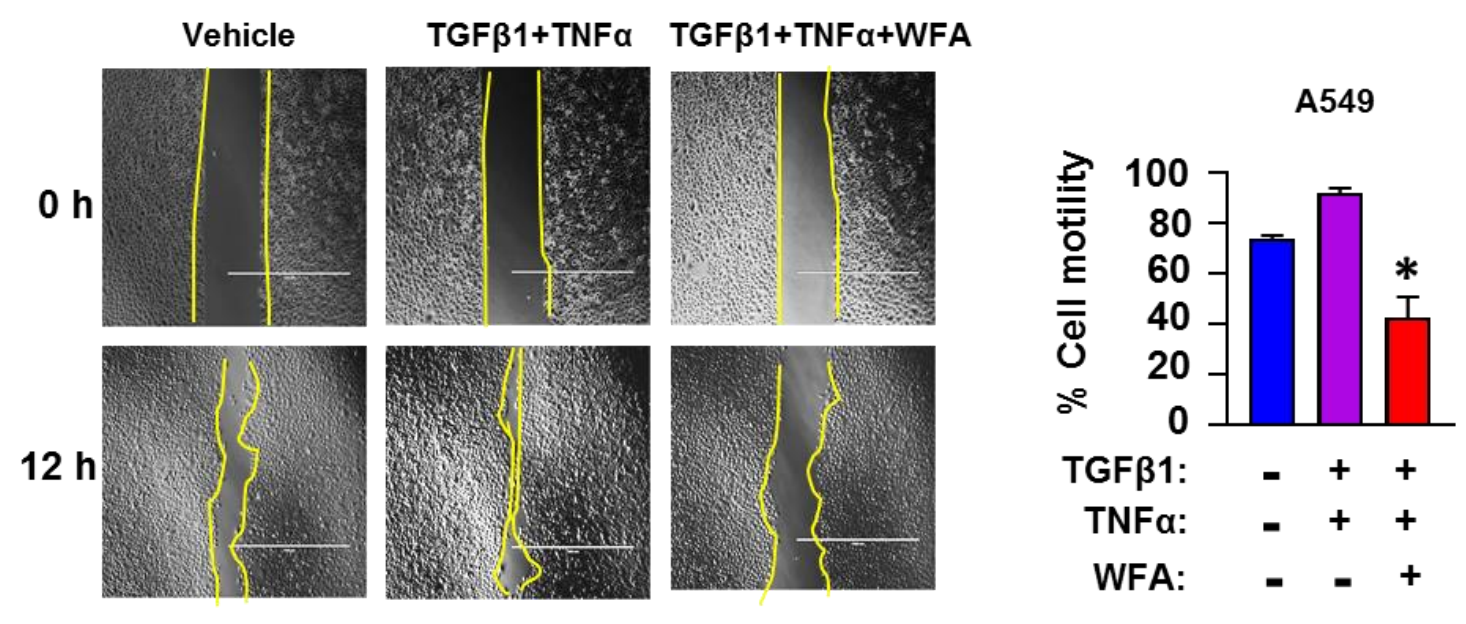

b
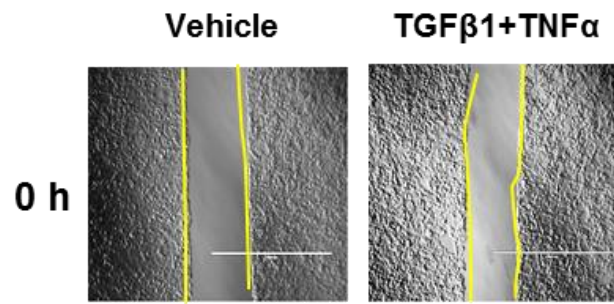

TGF $\beta 1+$ TNF $\alpha+$ WFA
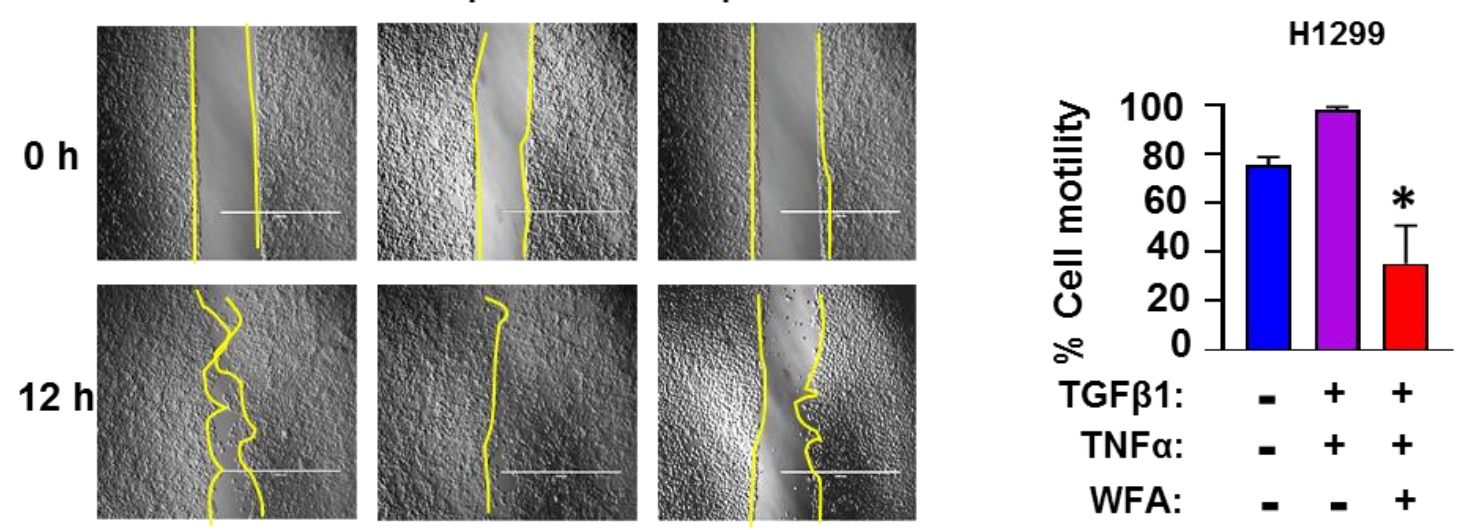

Fig 3.4. WFA suppressed TGFß1/TNF $\alpha$-induced motility of A549 and H1299 cells. Wound healing assay was used to measure the motility of the cells in the presence $0.5 \mu \mathrm{M}$ WFA and a combination of TGF $\beta 1(5 \mathrm{ng} / \mathrm{mL})$ or TNF $\alpha(25 \mathrm{ng} / \mathrm{mL})$. The figure shows that TGF $\beta 1 /$ TNF $\alpha$ significantly enhanced the motility of A549 (a), and H1299 (b) cells. Data are presented as percent migration (mean $\pm \mathrm{SD}$ ) by considering wound area at time $0 \mathrm{~h}$ as $100 \%$. $\left({ }^{*} \mathrm{p}<0.05\right)$ higher motility compared with vehicle-treated cells. 


\section{WFA inhibited TGF $\beta 1$-dependent Smad2/3 signaling in A549 and H1299 cells}

TGF $\beta 1$-induced EMT in tumor cells has been shown to occur mainly through the activation of Smad-dependent signaling pathways. Therefore, to assess whether WFA affected TGF $\beta$-induced Smad2/3 activation, cells were incubated with WFA at indicated concentrations and then stimulated with TGF $\beta 1(5 \mathrm{ng} / \mathrm{mL})$. The phosphorylation of Smad2/3 was assessed by immunofluorescence staining and western blot analysis. Confocal images (40x magnification) of A549 cells (Fig. 3.5a) shows probed for pSmad2/3 (green), F-actin (red) and nuclei (blue). These images show that TGF $\beta 1$ increased the levels of $\mathrm{p}-\mathrm{Smad} 2 / 3$ and its nuclear localization when compared to vehicle-

treated cells. However, pre-incubation of cells with WFA prior to TGF $\beta 1$ stimulation, the phosphorylation and nuclear translocation of Smad2/3 was inhibited.

To validate the confocal data, we performed western blot analysis on whole cell, nuclear, and cytoplasmic lysates prepared from A549 and H1299 cells. As shown in (Fig.3.5b), we observed that WFA dose-dependently suppressed Smad2 and Smad3 phosphorylation and thus prevented their nuclear translocation. Moreover, it appears that WFA also decreased the levels of total Smad3 in a dose-dependent manner. Overall, the data presented here show that WFA inhibited TGF $\beta 1$-induced EMT at least in part via its regulation of Smad-dependent mechanisms. 
a
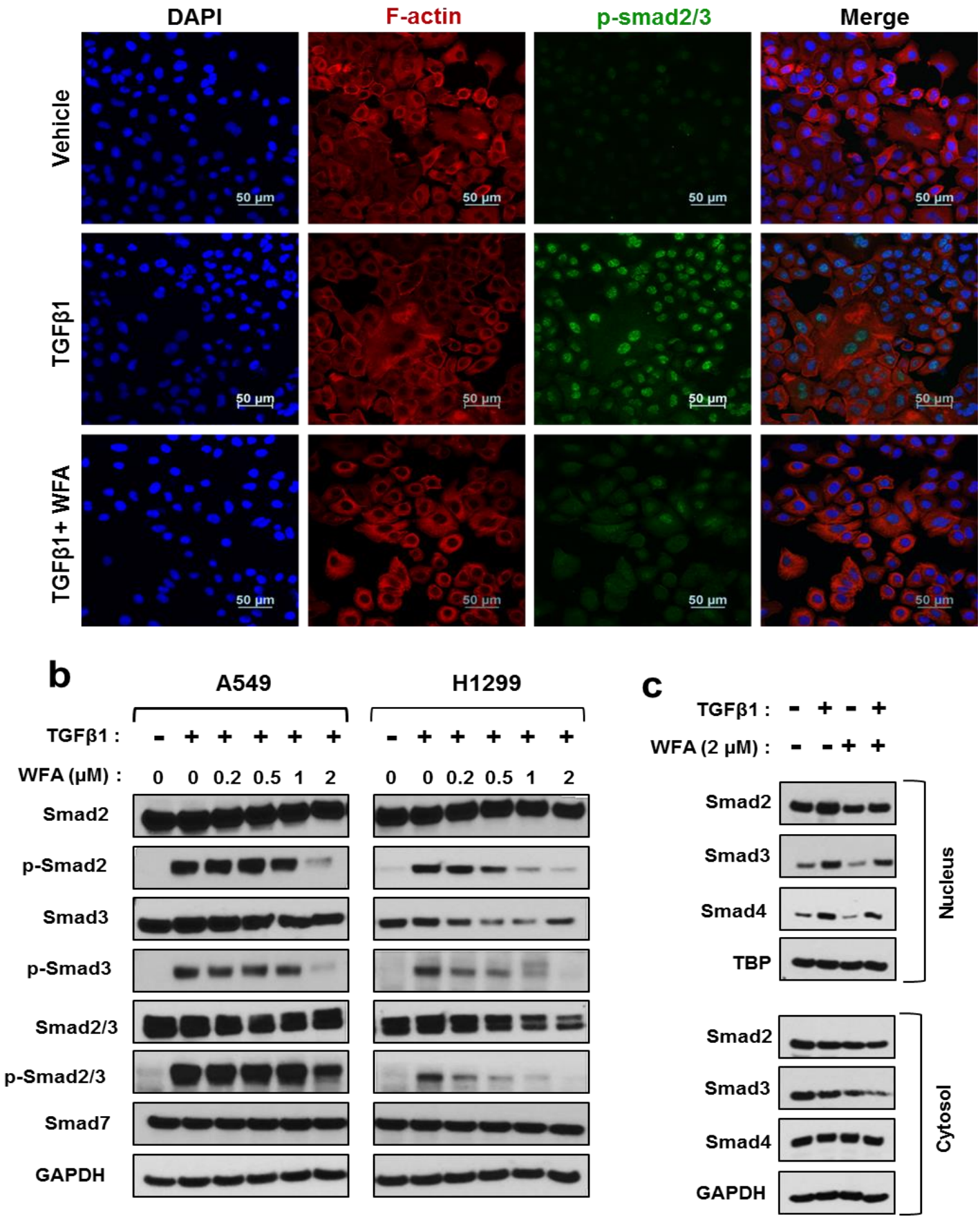

Fig 3.5. WFA inhibited TGFß1-dependent activation of Smad2/3 in A549 and H1299 cells. (a) Confocal images of A549 NSCLC cells depicting the inhibitory effect of WFA $(1.0 \mu \mathrm{M})$ on TGF $\beta 1(5 \mathrm{ng} / \mathrm{mL})$-induced Smad2/3 phosphorylation in A549 cells. (b) Western blot analysis of whole cell lysates depicting the dose-dependent inhibitory effect of WFA on the Smad2/3 signaling pathway (c) Western blot analysis of cytosolic and nuclear fractions from A549 cells showed decreased Smad2/3 phosphorylation and nuclear translocation in the presence of WFA. GAPDH was used the total protein loading control. 


\section{WFA inhibited TNF $\alpha$-induced NFkB signaling in A549 and H1299 cells.}

$\mathrm{TNF} \alpha$ stimulates aberrant $\mathrm{NF}-\kappa \mathrm{B}$ signaling pathways in tumor cells to regulate motility, migration, invasion, and chemo-resistance [97]. In the present study, we investigated whether WFA inhibited TNF $\alpha$-induced activation of NFאB in H1299 and A549 cells. As shown in Figure 6, TNF $\alpha$ stimulated the NF- $\kappa \mathrm{B}$ signaling pathway by inducing the phosphorylation and nuclear translocation of $\mathrm{NF}-\kappa \mathrm{B}(\mathrm{P} 65)$. As assessed by immunofluorescence staining (Fig. 3.6a), cells stimulated with TNF $\alpha$ had higher levels of $\mathrm{p}-\mathrm{NF}-\kappa \mathrm{B}$ than vehicle-treated controls. However, pre-incubation of cells with WFA significantly decreased the phosphorylation of $\mathrm{NF \kappa B}$. Western blot analysis (Fig. 3.6bc) of the whole-cell and cytosolic lysates indicates that TNF $\alpha$ alone increased the phosphorylation of $\mathrm{I} \kappa \mathrm{B} \alpha(\mathrm{p}-\mathrm{I} \kappa \mathrm{B} \alpha)$ and $\mathrm{NF} \kappa \mathrm{B}(\mathrm{p}-\mathrm{NF}-\kappa \mathrm{B})$. The phosphorylation of I $\mathrm{B}$ resulted in increased degradation of $\mathrm{I} \kappa \mathrm{B}$ and the nuclear translocation of NF- $\kappa \mathrm{B}(\mathrm{P} 65)$. However, WFA suppressed the TNF $\alpha$-dependent phosphorylation of NF- $\mathrm{B}(\mathrm{P} 65)$ and $\mathrm{I} \kappa \mathrm{B} \alpha$, the degradation of $\mathrm{I} \kappa \mathrm{B} \alpha$ and nuclear translocation of NF- $\kappa \mathrm{B}(\mathrm{P} 65)$. Interestingly,

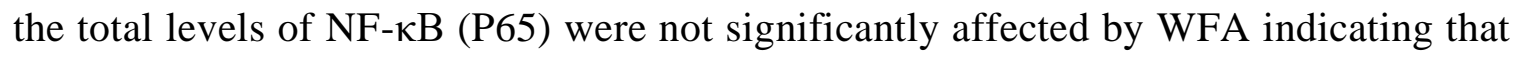
WFA inhibited NFאB signaling by regulation phosphorylation. By measuring the levels of total and phosphorylated IKK $\alpha / \beta$, we found that WFA inhibited $N F-\kappa B(P 65)$ by regulating the activity of IKK $\alpha / \beta$. Furthermore, we also investigated the effect of WFA on the transcriptional activity of NFKB (P65) using the electrophoretic mobility shift assay (EMSA). Our data (Fig. 3.6e) shows WFA decreased the binding of NFאB (p65) on to DNA in both A549 and H1299 cells. Overall, we demonstrate that WFA remarkably inhibited $T N F \alpha$-induced degradation of $I \kappa B$ resulting in inhibition of the

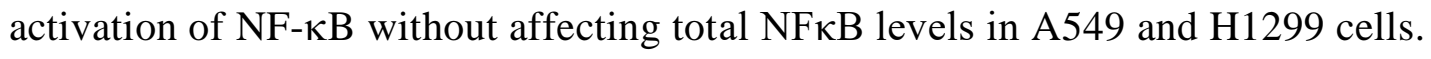



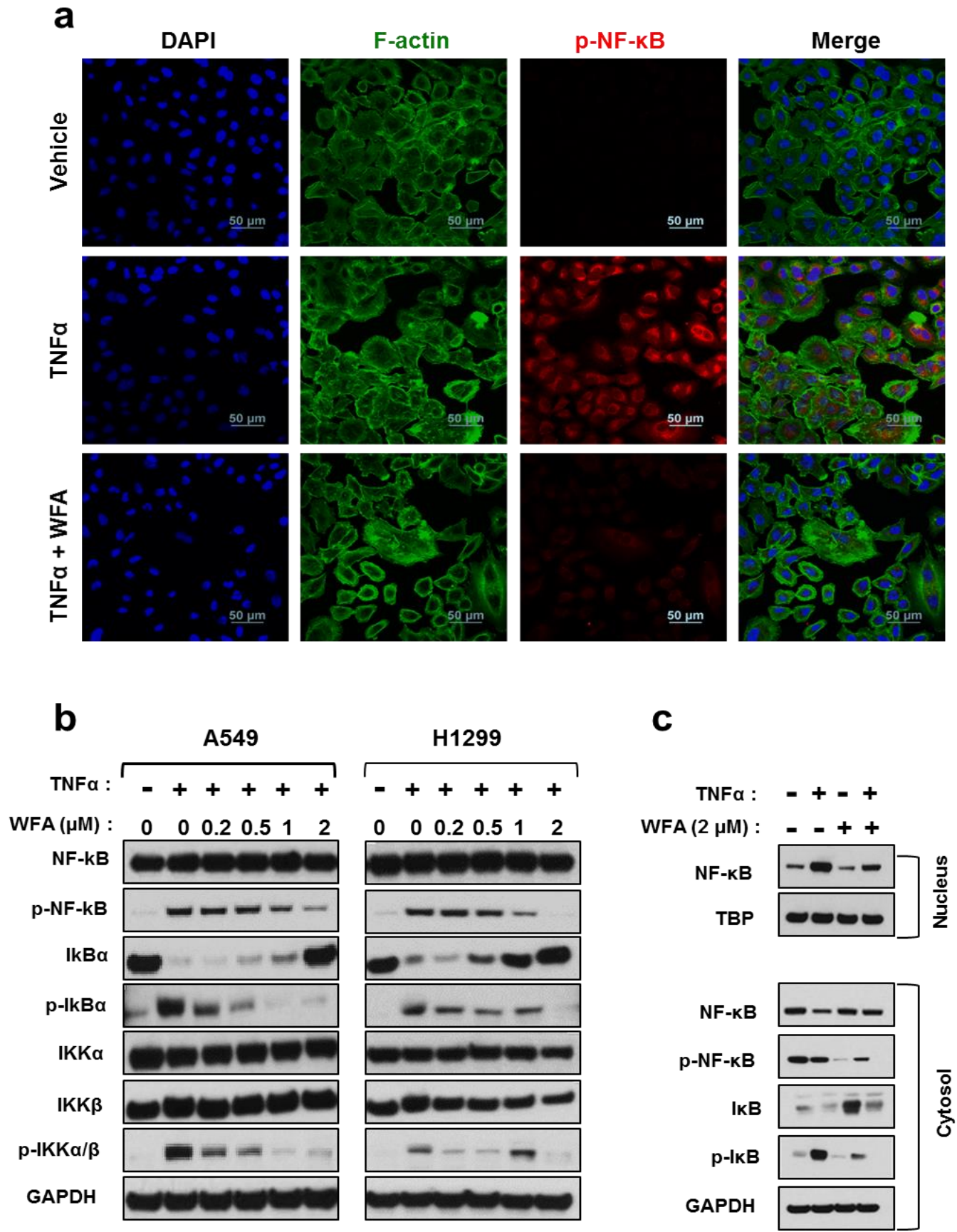

Fig 3.6. Inhibition of TNFa-induced NFKB activation in human NSCLC cells. (a) Confocal images (40x magnification) showing the levels of p-NF- $\mathrm{kB}$. (b) Western blot analysis depicting levels of $\mathrm{p}-\mathrm{NF} \kappa \mathrm{B}$, total NF-kB, $\mathrm{p}-\mathrm{I} \kappa \mathrm{B}$, and total I $\mathrm{\kappa B}$ in whole cell lysates following stimulation with $\mathrm{TNF} \alpha(25 \mathrm{ng} / \mathrm{mL}$ (c) Western blot analysis of nuclear and cytosolic cell extracts depicting levels of $\mathrm{NF} \kappa \mathrm{B}, \mathrm{p}-\mathrm{NF} \kappa \mathrm{B}, \mathrm{I} \kappa \mathrm{B} \alpha$, and $\mathrm{p}-\mathrm{I} \kappa \mathrm{B} \alpha$ following stimulation with TNF $\alpha$. 


\section{DISCUSSION}

Despite the recent advances in the treatment of NSCLC such as targeted therapies and immunotherapies, invasion and metastasis remain a major therapeutic challenge in NSCLC [81]. Recently, it has become clear that EMT plays a crucial role in the metastasis of NSCLC tumors and therefore has become an attractive drug target [90]. It has been shown that EMT induction causes tumor cells to become more migratory, invasive, and metastatic [92]. Additionally, EMT stimulates tumor cells to acquire stem cell-like properties and increases resistance to standard chemotherapeutic drugs [98]. Therefore, the importance of EMT during metastasis and in drug resistance of tumor cells has prompted the search for compounds that inhibit EMT in tumor cells as potential drugs for metastatic NSCLC [99]. Based on this hypothesis, we and others [31, 43, 96] have examined the

effects of WFA on experimentally induced EMT to demonstrate its potential antimetastatic properties.

WFA is a member of the withanolides, a large group of naturally occurring 28carbon-containing compounds [19]. The basic chemical structure of withanolides is composed of a steroidal framework attached to a lactone ring, and are also referred to as 'steroidal-lactones' [20]. In WFA (Figure 1A), there are five functional groups including; an unsaturated ketone ring, 2 hydroxyl groups, an epoxide ring, a 6-carbon lactone ring, and an unsaturated carbonyl group [27, 28]. It has been shown that these five functional groups enable WFA to interact with several molecular targets and account for the diverse biological activities of WFA including its anti-cancer properties [22]. Multiple lines of experimental evidence from in vitro and in vivo studies show that WFA is a highly efficacious anti-tumor molecule that displays both cytotoxic activities against multiple 
cancer cells $[37,64,100]$. Recently, the effects of WFA on tumor cell migration, invasion, and metastasis [22] have gained a lot of attention and have stimulated interest in investigating the anti-metastatic properties of WFA. Importantly, unlike common cytotoxic chemotherapeutic drugs that have been shown to induce EMT [101], WFA, on the other hand, suppresses EMT [31, 43, 96, 102] and therefore has the capability to prevent metastasis and drug resistance in multiple cancers.

Earlier studies have shown that sub-cytotoxic doses inhibit EMT induction in the breast, ovarian, and mammalian epithelial cells $[96,103]$. In fact, detailed molecular evidence showed that WFA binds to and causes the degradation of the EMT-related intermediate filament protein, vimentin [31]. Similarly, our study highlights the role of WFA in regulating EMT and metastasis in tumor cells. Initially, we determined the cytotoxicity of WFA on anchorage-dependent growth and proliferation of A549 and H1299 cells. As reported elsewhere [64], our findings show remarkable cytotoxicity of WFA on the anchorage-dependent proliferation of NSCLC cells. Notably, the highly invasive H1299 cells were more sensitive to WFA than the moderately invasive A549 cells. This finding suggests and strengthens the hypothesis that WFA is likely more potent against the highly metastatic tumors which are incidentally found in the majority of NSCLC patients.

During the EMT process, tumor cells lose cell to cell contacts, become more invasive and resistant to cytotoxic drugs [104]. At the molecular level, it is reported that the loss of the epithelial marker E-cadherin coupled with an increase in mesenchymal markers such as vimentin and N-cadherin are the hallmarks of EMT [90]. Furthermore, EMT pathways are regulated by various extracellular signals including cytokines in the tumor microenvironment such as TGF $\beta 1$ and TNF $\alpha[104,105]$. Experimentally, EMT 
induction in tumor cells has been modeled by culturing cells in serum-free media containing TGF $\beta 1$ and/or TNF $\alpha$ [92]. Using this model, we successfully induced EMT in A549 and H1299 cells using TGF $\beta 1$ and TNF $\alpha$. In agreement with previous studies [106], our findings data shows synergistic cooperation between TGF $\beta 1$ and TNF $\alpha$ on E-cadherin repression. More importantly, pre-incubation of cells with WFA prior to EMT induction prevented the loss of E-cadherin or the upregulation of $\mathrm{N}$-cadherin. Together with previous data [31, 96, 107], our study demonstrates that WFA is a powerful suppressor of EMT induction and further highlights the anti-metastatic effects of WFA.

The molecular mechanisms underlying EMT are complex but the transcription factor Snail has been shown to be a key regulator of EMT induction in NSCLC cells [105]. Specifically, Snail binds to DNA and causes the repression of E-cadherin expression while upregulating EMT-related proteins such as vimentin, N-cadherin, and fibronectin [92, 98, 105]. Snail expression and function are regulated by extracellular signals such as TGF $\beta 1$ [90, 92] and TNF $\alpha$ [106] via Smad- and NF-kB-dependent signaling pathways, respectively. Moreover, simultaneous activation of these two pathways synergistically increases the expression Snail in A549 cells [106, 108, 109]. In agreement with this observation, we demonstrated that a combination of TGF $\beta 1$ and TNF $\alpha$ synergistically increased the expression of Snail. Because Snail represses E-cadherin expression, this finding explains the observed synergistic interaction between TGF $\beta 1$ and TNF $\alpha$ on EMT induction as indicated by the enhanced loss of E-cadherin and upregulation of N-cadherin. However, WFA pre-incubation abrogated the cytokine-dependent increase in Snail expression, and further prevented the loss of E-cadherin thus preventing EMT induction. Overall, these findings suggest that the observed inhibition of EMT induction by WFA 
occurs at least in part due to the regulation of Snail expression by inhibiting Smad and/or $\mathrm{NF}-\kappa \mathrm{B}$ signaling pathways.

To further understand the potential mechanisms underlying the inhibitory effects of WFA on TGF $\beta 1 / \mathrm{TNF} \alpha$-dependent EMT induction, we explored Smad and NF- $\mathrm{B}$ signaling pathways. Smad2 and Smad3 belong to a family of transcription factors that exist as a Smad2/3 dimer in the cytoplasm in the inactive (dephosphorylated) form. However, following TGF $\beta 1$ stimulation, these are phosphorylation, then bind to another cytosolic protein called Smad4 and translocate to the nucleus where they stimulate the expression of Snail [105]. Therefore, to determine whether WFA inhibits the activation of Smad signaling, we measured the levels of total and phosphorylated Smad2, Smad3, and Smad4 in whole-cell, cytoplasm, and nucleus. Although WFA has already been shown to inhibit Smad2/3 phosphorylation [107], our study provides additional evidence on the multiple effects of WFA on Smad signaling. WFA inhibited the phosphorylation of both Smad2 and Smad3, and thus prevented their nuclear translocation. Additionally, it appears that WFA also targeted Smad3 and caused its degradation.

Several published studies have shown that WFA is a potent anti-inflammatory activity and has been extensively studied for its effects NF- $\kappa B$ signaling mechanisms [22, $100,110,111]$. In tumor cells, it is known that $\mathrm{TNF} \alpha$ activates the NF- $\kappa \mathrm{B}$ signaling to stimulate EMT induction, cell survival, proliferation, migration, and chemo-resistance [97, 109]. Mechanistically, the activation of NFKB involves two major events; (1) IKK $\alpha / \beta$ dependent phosphorylation and subsequent degradation of $\mathrm{I} \kappa \mathrm{B} \alpha$, the inhibitor of $\mathrm{NF}-\kappa \mathrm{B}$ (P65), and (2) the phosphorylation and nuclear translocation of NF- $\kappa \mathrm{B}$ (P65). Data from previous studies [110] conducted on fibrosarcoma and human embryonic kidneys cells 
indicated that WFA regulated NF- $\kappa B$ signaling by inhibiting the kinase activity of IKK $\alpha / \beta$. This resulted in decreased phosphorylation and degradation of $\mathrm{I} \mathrm{KB} \alpha$, the phosphorylation and nuclear translocation of NF- $\mathrm{KB}$ (P65). Because TNF $\alpha$ is known to induce EMT via the NF- $\kappa B$ pathway, we also assessed the effect of WFA on NF- $\kappa B$ signaling. In agreement with previous findings, we also observed dose-dependent inhibition of TNF $\alpha$-dependent activation of NF-kB signaling in both A549 and H1299 cells. The phosphorylation and

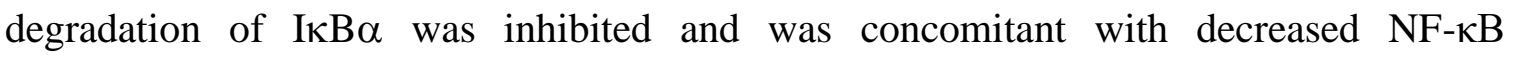
phosphorylation, nuclear translocation, and binding to DNA. These findings provide additional supporting evidence of the inhibitory effect of WFA on NFKB signaling via interference with IKK $\alpha / \beta$ kinase activity.

In conclusion, using a combination of TGF $\beta 1$ and TNF $\alpha$ to experimentally induce EMT, our study demonstrates the inhibitory of WFA on EMT induction in A549 and H1299 NSCLC tumor cells. Although detailed molecular mechanisms have not been elucidated, our data provides a basis for the conclusion that WFA regulates EMT via the regulation of Smad and NFkB signaling pathways. Together, the findings presented here and in previous studies suggest that WFA is a promising antitumor agent that has therapeutic potential in the treatment of metastatic NSCLC cancer. Additionally, our observation that WFA was more potent against the highly invasive H1299 cells than the moderately invasive A549 cell line, further strengthens this hypothesis. 


\section{CHAPTER FOUR}

\section{REPEATED DOSING TOXICITY AND PHARMACOKINETICS OF WITHAFERIN A IN SPRAGUE DAWLEY (SD) RATS}

\section{INTRODUCTION}

Pharmacokinetics defines the processes of absorption, distribution, metabolism, and the excretion of drugs in the body [112]. Currently, systemic chemotherapy using cytotoxic agents remains the mainstay in the treatment of advanced NSCLC without targetable mutations [9]. In NSCLC, multiple high doses of cytotoxic drugs are often used to achieve the desired therapeutic response [16]. Many of the drugs used have a very narrow therapeutic window and require precise therapeutic dose monitoring to minimize the risks for toxicity. Traditionally, the dosing of NSCLC patients with various standard chemotherapeutic agents is based on the assumption of a linear relationship between body surface area (BSA) and clearance (CL) or volume of distribution (Vd) [113]. However, remultiple clinical trials $[112,114]$ have shown that this is not true and that most anticancer agents show interindividual pharmacokinetic variabilities. For example, systemic exposure (AUC) after standard doses of paclitaxel varies by up to 10 -fold between patients [112]. Moreover, NSCLC patient populations are often very diverse and complex, which creates the need for explicit drug dosing recommendations. 
The high variability of the pharmacokinetic properties of cytotoxic anticancer drugs in patient populations make it very difficult for oncologists to predict individual clinical responses in patients [112]. Moreover, with variable pharmacokinetics comes an increased risk of both toxicity and sub-therapeutic dosing, leading to compromised clinical efficacy [114]. On the other hand, if a drug has well-characterized pharmacokinetic properties, it is possible to decrease interindividual pharmacokinetic differences, minimize toxicity and enhance clinical efficacy by tailoring doses to the individual patient (the so called individualized dosing) [12, 48]. Therefore, to enhance clinical efficacy, it is important to characterize the toxicity and pharmacokinetics of new anticancer agents early enough in the drug discovery process to provide a framework for designing safe and efficacious dosing schedules.

Withanolides are a novel class of bioactive compounds that have shown multiple pharmacological activities [115]. Withaferin A (WFA) is the most prominent member of this class of compounds and has emerged as a potential therapeutic agent for cancer [22, 24]. Chemically, WFA is a plant-derived steroidal-lactone that was first isolated from the alcoholic extracts of the Ayurvedic medicinal plant Withania somnifera [23, 24, 26]. In literature, there is a growing body of in vitro and in vivo preclinical data to demonstrable the efficacy of WFA against NSCLC [62, 83], breast [31, 35], cervical [66], prostate [39, 94], pancreatic [67], renal [116], B-cell lymphoma [68] and ovarian cancers [43]. Mechanistically, it has been shown that WFA targets heat shock proteins [117], vimentin [31], NFkB [83], IKK $\beta$-kinase [118], STAT3 [22], Blc2, cyclins, EGFR, and PAR-4 [30, 94]. Together, these varied molecular targets allow WFA to disrupt multiple cellular processes such as proliferation, migration, invasion, and metastasis that are critical in the 
tumorigenesis of NSCLC cells [22]. Unfortunately, despite its demonstrable antitumor efficacy in multiple models, WFA is currently stuck at the preclinical testing stage mainly due to the lack of toxicity and pharmacokinetics data. There is very limited reliable data on important disposition parameters of WFA such as; metabolic stability, plasma protein binding, erythrocyte partitioning, excretion properties, and clearance.

In the present study, the objectives were to; (1) determine the potential repeated dose toxicity, and (2) determine the preclinical pharmacokinetic properties of WFA. Briefly, the sub-chronic toxicity of WFA was assessed for three doses $(4,8,12 \mathrm{mg} / \mathrm{kg})$ of WFA in male SD rats following 28-days of repeated oral dosing. The toxic endpoints consisted of an evaluation of changes in total body weight gain, food intake, hematological and biochemical parameters. On this regard, our data indicate that up to $12 \mathrm{mg} / \mathrm{kg}$ of WFA was safe and well-tolerated in male SD rats after oral administration. No significant changes in total body weight, body weight gain, hematological and biochemical parameters were observed. To study the pharmacokinetics of WFA, we first developed a highly sensitive analytical method for the quantification of WFA in plasma and tissues. In this method, we used HPLC as the chromatographic separation technique coupled to MS/MS for detection and quantitation. This analytical method was successfully developed and applied to determine the pharmacokinetic properties of WFA after administration of a single bolus (i.v.) dose (10mg/kg) in male SD rats. In agreement with previous studies[31], WFA displayed linear pharmacokinetics, with very rapid distribution and elimination from plasma, and the concentrations fell below the detection limit within 2 h. Further, WFA displayed high plasma protein binding and rapid intrinsic clearance in liver microsomes. 


\section{MATERIALS AND METHODS}

\section{Chemicals and reagents}

Withaferin A (Mol. Wt 470.61, purity > 95\% HPLC) was kindly provided by 3P Biotechnologies (Louisville, KY, USA). Dimethyl sulfoxide (DMSO, Cat \#13390) was purchased from Electron Microscopy Sciences (Hatfield, PA, USA). Pooled SD rat liver microsomes were purchased from ThermoFisher (Kansas City, KS). Prednisolone analytical standard (Mol. Wt. 360.44, Cat\#46656) was purchased from Sigma (SigmaAldrich, St Louis, MO, United States). In addition, acetonitrile, methanol, and ultrapure HPLC-grade water were purchased from Sigma Aldrich (St. Louis, MO, USA).

\section{Animals}

Male SAS Sprague Dawley (SD) rats (150-200 g) were purchased from Charles River Laboratories. These rats were housed individually or as a maximum of 3 rats/cage in a temperature-controlled room $\left(25^{\circ} \mathrm{C}\right)$ with a 12 -h light/dark cycle. The rats were allowed access to purified AIN-93M diet and water ad libitum The research protocols involving the use of animals were approved in advance by the Institutional Animal Care and Use Committee (IACUC\#17041).

\section{Sub-chronic toxicity of WFA in male SAS SD rats}

In this study, a total of 20 male SAS SD rats were used to evaluate the potential toxicity of WFA following repeated oral dosing. Prior to the initiation of treatments, the rats were allowed a minimum of 7-days acclimation period and then randomly divided into 4 experimental groups ( $n=5 /$ group). To the first group, we administered a drug-free vehicle (0.1 M $\beta$-hydroxypropyl cyclodextrin) in an equivalent volume with drug-treated rats. In 
the remaining 3 groups, rats were administered with 3 escalating doses of WFA $(4,8,12$ $\mathrm{mg} / \mathrm{kg}$ ). For the vehicle and all experimental groups, the rats were orally administered with treatments three times per week for a total of four weeks (28-days). To assess the potential toxicity of the treatments, several toxic endpoints were determined. Firstly, we observed the rats daily for clinical signs of toxicity such as overall activity and isolation, breathing rate, fur coat, eye color, and discharge. Before dosing (3 times/week), the body weights for all rats as well as the amount of food per cage were recorded. After 28-days, the study was terminated and all the surviving rats were humanely sacrificed by $\mathrm{CO}_{2}$ asphyxiation. All blood from the carcasses was collected via cardiac puncture and selected organs (liver, kidneys, lungs, spleen, brain, pancreas, gut, heart) were dissected out. The blood samples for each rat were divided into two sub-samples and separately subjected to hematological and biochemical clinical investigations (Antech Diagnostics). Some of the hematological parameters evaluated included; total white blood cell counts (WBC), neutrophils, lymphocytes, monocytes, eosinophils, basophils, total erythrocyte counts (RBC), hemoglobin $(\mathrm{Hb})$, hematocrit (HCT), and platelet count (MPV). For serum clinical chemistry, the following parameters were determined: albumin, alkaline phosphatase (ALP), aspartate aminotransferase (AST), alanine aminotransferase (ALT), bilirubin direct, bilirubin total, creatinine $(\mathrm{Cr})$, total protein, and blood urea nitrogen (BUN). Furthermore, the selected tissues were weighted and random samples for each organ were fixed in $10 \%$ neutral buffered formalin and sent for histopathological analysis.: For each body organ removed, the absolute organ weight (g) was used to calculate the relative organ weight with respect to the total body weight of each rat. 


\section{HPLC-MS/MS conditions for analysis of WFA}

The detection and quantitative analysis of WFA were performed on an Agilent 6550 iFunnel Q-TOF with Agilent JetStream equipped with an Agilent 1290 Infinity uHPLC (Agilent Technologies, Santa Clara, CA, USA). For each sample run, 6 ul of sample was injected onto a Zorbax Extend C18, 2.1 x $50 \mathrm{~mm}, 1.8$-micron column(Agilent) kept at $40^{\circ}$ C. The mobile phase was; A: $0.1 \%$ formic acid in water, B: acetonitrile. The mobile phase gradient: $0-4 \min 80 \%$ A to $5 \% \mathrm{~A}, 4-10 \mathrm{~min}$ held at $5 \% \mathrm{~A}, 10-12 \min 5 \%$ A to $80 \% \mathrm{~A}$. The eluant was diverted to waste for the first $4 \mathrm{~min}$ of the run. High-resolution spectra were obtained in the positive mode with a source gas temperature of $225^{\circ} \mathrm{C}$, sheath gas temperature of $375^{\circ} \mathrm{C}$ with a flow rate of $11 \mathrm{~L} / \mathrm{min}$. The MS spectra were obtained in the mass range of $300-600 \mathrm{~m} / \mathrm{z}$ and $90-600 \mathrm{~m} / \mathrm{z}$ in the MS/MS mode. We used a fixed collision energy of 20 for targeted MS/MS analysis. The precursors chosen were m/z 471 for WFA and m/z 361 for the IS. For WFA, m/z 95 was used for quantification and m/z 281 and 299 were used as qualifiers. For the IS (prednisolone), m/z 147 was used for quantification with ions 325 and 343 used as qualifiers.

\section{Preparation of WFA Standard Solutions}

Stock solutions of WFA and prednisolone were prepared by dissolving known amounts of each agent in methanol. Standard solutions of WFA and prednisolone for HPLC-UV analysis were diluted to final concentrations of $0.3-40 \mu \mathrm{g} / \mathrm{mL}$ while for HPLCMS/MS analysis, the standards were diluted to final concentrations of $0.5-5000 \mathrm{ng} / \mathrm{mL}$. Calibrations standards were prepared by spiking known amounts of WFA in blank plasma or tissue matrix. 


\section{Plasma and tissue sample preparations}

To determine WFA concentration in plasma, aliquots $(100 \mu \mathrm{L})$ of plasma were mixed with $100 \mu \mathrm{L}$ internal standard solution (100 $\mathrm{ng} / \mathrm{mL}$ prednisolone) followed by the addition of $800 \mu \mathrm{L}$ of ice-cold acetonitrile. The mixture was vortexed vigorously for $1 \mathrm{~min}$ and then centrifuged at $10,000 \times \mathrm{g}$ for $10 \mathrm{~min}$ at $4{ }^{\circ} \mathrm{C}$. The supernatants were carefully transferred into $2 \mathrm{~mL}$ silanized glass tubes and dried completely under vacuum. The residues were reconstituted in $100 \mu \mathrm{L}$ of methanol, filtered and transferred to $1.5 \mathrm{~mL}$ HPLC vial for analysis of the concentrations of WFA by HPLC-MS/MS method. Similarly, to prepare tissue samples, about $1 \mathrm{~g}$ of tissue was homogenized in $3 \mathrm{~mL}$ of ice-cold $0.9 \%$ saline solution. Then, for every $1 \mathrm{~mL}$ of tissue homogenate, $100 \mu \mathrm{L}$ of IS solution was added followed by the addition of $4 \mathrm{~mL}$ of ice-cold acetonitrile, vortexed vigorously for $1 \mathrm{~min}$ and then centrifuged at $8,000 \mathrm{x} g$ for $10 \mathrm{~min}$. The supernatant was carefully separated from the tissue debris, transferred to a new tube using a pipette and dried completely under vacuum. The residues were reconstituted in $1 \mathrm{~mL}$ of acetonitrile: water (8:2) and loaded on to Captiva-EMR lipid SPE cartridge (Agilent Technologies). The samples were allowed to flow under gravity and followed by application vacuum to dry the cartridges. The eluent was dried completely and then reconstituted in $100 \mu \mathrm{L}$ of methanol prior to HPLC-MS/MS analysis.

\section{Plasma pharmacokinetics of WFA in male SD rats}

To determine the plasma pharmacokinetic properties of WFA, male SAS SD rats were cannulated in the right jugular veins with polyethylene cannulae. The surgery to insert cannulae was performed on rats at Charles River Laboratories prior to the purchase and 
delivery of the rats. Following surgery, the rats were allowed to recover for one day and then shipped overnight to the study site. In addition, the rats were allowed one more week of acclimation period during which they were allowed free access to water and purified AIN-93M diet ad libtum. On the day of the study, rats were administered with $10 \mathrm{mg} / \mathrm{kg}$ ( $1 \mathrm{mg} / \mathrm{mL}$ in $0.1 \mathrm{M} \beta$-hydroxypropyl cyclodextrin) as a single bolus intravenous dose $(<1$ min injection) via the lateral tail vein. Serial blood (200 $\mu \mathrm{L} /$ time point) samples were collected at $0,5,15,30,45,60,90,120,240,360$ and 480 minutes into heparinized tubes. The blood samples were processed to obtain plasma by centrifuging for $15 \mathrm{~min}$ at $2000 \mathrm{x}$ $\mathrm{g}$ and stored at $-80^{\circ} \mathrm{C}$ until analysis for WFA concentration using HPLC-MS/MS method. The concentration-time data of WFA in male SAS SD rats were subjected to noncompartmental analysis (NCA) using Pheonix WinNonLin 8.1 software (Pharsight, CA, USA). The following parameters calculated: $\mathrm{AUC}_{0-\mathrm{t}}, \mathrm{AUC}_{0-\infty}, \mathrm{t}_{1 / 2}$, total plasma clearance (CL), mean residence time (MRT).

\section{Protein binding of WFA in SAS SD rat plasma}

The binding of anticancer drugs to plasma proteins significantly affects their disposition kinetics and therapeutic efficacy [112]. Therefore, the objective of this study was to determine the fraction of WFA bound to plasma proteins in SAS SD rat plasma. Protein binding of WFA in freshly collected plasma was determined by ultracentrifugation method for separating bound and unbound drugs using the Centrifree Ultrafiltration device (Millipore, USA) with a 3000-molecular cut-off. Initially, we determined that the nonspecific adsorption of WFA on to the filter membrane by dissolving known amounts of WFA in PBS (5\% DMSO) and then determining the concentration of WFA before and after 
centrifugation. Then, for plasma protein binding capacity, aliquots of freshly collected plasma $(500 \mu \mathrm{L}) \mathrm{SD}$ rat plasma were spiked with known amounts of WFA in triplicates. The samples were thoroughly mixed and incubated at $37^{\circ} \mathrm{C}$ for $30 \mathrm{~min}$. The samples were transferred to the Centrifree Ultracentrifugation device and centrifuged at $3000 \mathrm{xg}$ for 20 min. After centrifugation, the concentration of WFA was determined in the pre- and post centrifugation samples (ultrafiltrate). The unbound fraction of $\left(f_{u}\right)$ of WFA was calculated as: $f_{u}=($ concentration of drug in ultra-filtrate/concentration in a pre-centrifuged sample $) \mathrm{x}$ $100 \%$.

\section{The stability of WFA in SD rat liver microsomes}

In this study, pooled frozen samples of SD rat liver microsomes $(20 \mathrm{mg} / \mathrm{ml})$ were purchased from ThermoFisher Scientific (Cat \# RTMCPL). To determine the stability of WFA in microsomes, in a total reaction volume of $1000 \mu \mathrm{L}$, we used $25 \mu \mathrm{L}$ of $20 \mathrm{mg} / \mathrm{mL}$ commercial liver microsomes, $4 \mu \mathrm{L}$ of drugs $(0.01,0.1$ and $1 \mu \mathrm{g} / \mathrm{ml}$ in methanol, $871 \mu \mathrm{L}$ of $0.1 \mathrm{M}$ phosphate buffer (pH 7.4) and mixed using a shaker for 5 min at $37^{\circ} \mathrm{C}$. Reactions were initiated by adding $100 \mu \mathrm{L}$ of $10 \mathrm{mM}$ NADPH solution to the mixture, and $100 \mu \mathrm{L}$ of the reaction solution will be collected at different time points $(0,5,15,30,60,90$ and 120 minutes). At each time point, the enzymatic reactions were stopped by the addition of 4 volumes of cold acetonitrile to precipitate the proteins. The samples were dried under vacuum and reconstituted in $100 \mu \mathrm{L}$ of methanol and analyzed for WFA concentration by HPLC-MS/MS. The concentrations of WFA at the start $(\mathrm{t}=0)$ were considered as reference and used to calculate percent remaining WFA with respect to $t=0$. The WFA/IS peak area ratios were plotted against incubation time curves ton estimate the in vitro half-life $\left(\mathrm{t}_{1 / 2}\right)$. 
The apparent intrinsic hepatic clearance was calculated as previously described [119] using the following formula:

$$
\begin{aligned}
& \text { Elimination rate constant }(\mathrm{k})=(\text {-gradient }) \\
& \text { Half life }\left(\mathrm{t}_{1 / 2}\right), \min =\frac{0.693}{\mathrm{k}} \\
& \mathrm{V}(\mu \mathrm{L} / \mathrm{mg})=\frac{\text { Volume of incubation }(\mu \mathrm{L})}{\text { Protein in the incubation }(\mathrm{mg})} \\
& \text { Intrinsic Clearance }\left(\mathrm{CL}_{\text {int }}\right)(\mu \mathrm{L} / \mathrm{min} / \mathrm{mg} \text { protein })=\frac{\mathrm{V} \times 0.693}{\mathrm{t}_{1 / 2}}
\end{aligned}
$$

Hepatic intrinsic clearance $\left(\mathrm{CL}_{\mathrm{in}, \mathrm{app}}\right)=\mathrm{CL}_{\text {microsome }} \mathrm{x}$ [microsome weight $(\mathrm{g}) /$ liver weight (g)] x [Liver weight $(\mathrm{g}) /$ body weight $(\mathrm{kg})]$

\section{Statistical analysis}

Statistical analysis was performed using GraphPad Prism 8.0 (Sandiego, CA, USA). Plasma concentration-time data was subjected to non-compartmental analysis (NCA) using the intravenous bolus model in Phoenix WinNonLin 8.1 (Pharsight, NC, USA). The pharmacokinetic parameters for WFA were generated from individual serial concentrations. The data are presented as means \pm standard deviation (SD). Where applicable, data comparisons between two groups were made using Student's independentsamples $t$-test and one-way analysis of variance (ANOVA). Statistical significance was set to be $p<0.05$. 


\section{RESULTS}

\section{Potential sub-chronic toxicity of WFA in male SD rats}

In this study, we evaluated the safety of repeated dosing of $4,8,12 \mathrm{mg} / \mathrm{kg}$ WFA to male SD rats for 28 days. All the rats were dosed succesifully with WFA for the 28 day period. None of the rats in all the 3 dose groups showed any signs of acute toxicity or morbidity during the study period. More importantly, no mortality of rats in either vehicle or experimental groups was observed at any of the 3 dose levels of WFA. Comparatively, there was a slight dose-dependent but non-significant increase in the overall body of rats dosed with WFA when compared to vehicle-treated rats over the 28-day period (Fig. 4.1a). This was also the case when we calculated the percent body weight gain in the groups of rats treated with WFA (Fig. 4.1b). However, while the percent body weight gains were dose-dependent, all the calculated average weekly percent body weight gains were statistically non-significant when compared to the vehicle-treated rats. Interestingly, data from the food intake studies indicated an increase food uptake among the rats that received WFA. Therefore, the observed dose-dependent increase in the body weights of rats that were treated with WFA was correlated with the increase in food intake (Fig. 4.1c). Overall, neither the changes in body weight gain nor food intake were statistically significant.

On dosing day 28 day, the toxicity study was terminated and all the rats were humanely sacrificed by asphyxiation under $\mathrm{CO}_{2}$ asphyxiation. Blood and body organs were collected at necropsy to evaluate systemic and histopathologic toxicity. There were no significant changes in percent body weight ratios for liver (Fig. 4.1d), lungs (Fig. 4.1e), kidneys (Fig. 4.1f) and spleen (Fig. 4.1g) of rats treated with WFA when compared to the 
vehicle control group. Analysis of whole-blood samples for complete blood count (CBC) showed no significant changes in WBC (Fig. 4.2a), neutrophils (Fig. 4.2b), lymphocytes (Fig. 4.2c) or monocytes (Fig. 4.2d). Similarly, the RBC (Fig. 4.2e), hemoglobin (Fig. 4.2f), hematocrit (Fig. 4.2g) or total platelet counts (Fig. 4.2h) were all comparable in the WFA treated rats and vehicle-treated group. Serum biochemical analysis did not show any significant changes in liver and renal function parameters. Total protein, albumin, blood urea nitrogen (BUN), creatinine and total electrolyte concentrations (sodium, potassium, chloride, and calcium) were non-signifcant (Fig. 4.3a). The enzymes AST, ALT, ALP, GGT were all compared in the WFA and vehicle treatment groups. Histologic examination of H\&E sections of various organs revealed no visible microscopic changes in cellular architecture, evidence of tissue necrosis or inflammation in liver, kidneys, and lungs. Together, these findings show that repeated oral dosing of up to $12 \mathrm{mg} / \mathrm{kg}$ of WFA did not cause any major gross or systemic toxicity and was well tolerated in male SD rats. 

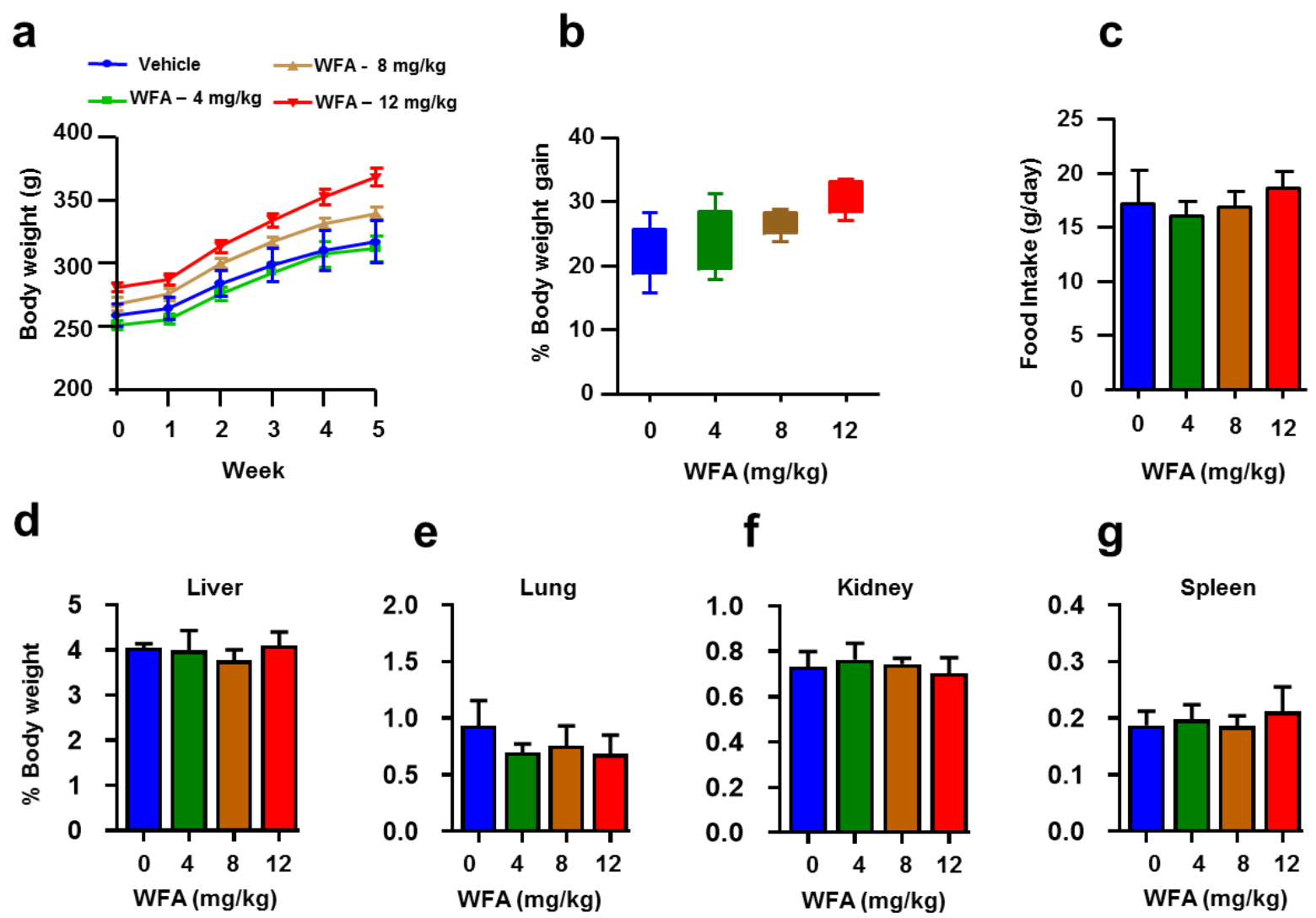

Fig 4.1. Effect of WFA on body and organ weight. Male SD rats were orally administered with vehicle (0.1 M $\beta$-hydroxypropyl cyclodextrin) or WFA $(4,8,12 \mathrm{mg} / \mathrm{kg}$ ) three times per week. (a) Mean weekly body weights, (b) Percent body weight gains, and (c) Mean daily food intake. Terminal organ weight ratios were calculated for each animal and presented as mean percent ratios for (d) liver, (e) lung, (f) kidneys, and (g) spleen. All data were presented as mean \pm SD for each group of rats of 3 technical replicates. *p<0.05 when compared with vehicle control. 


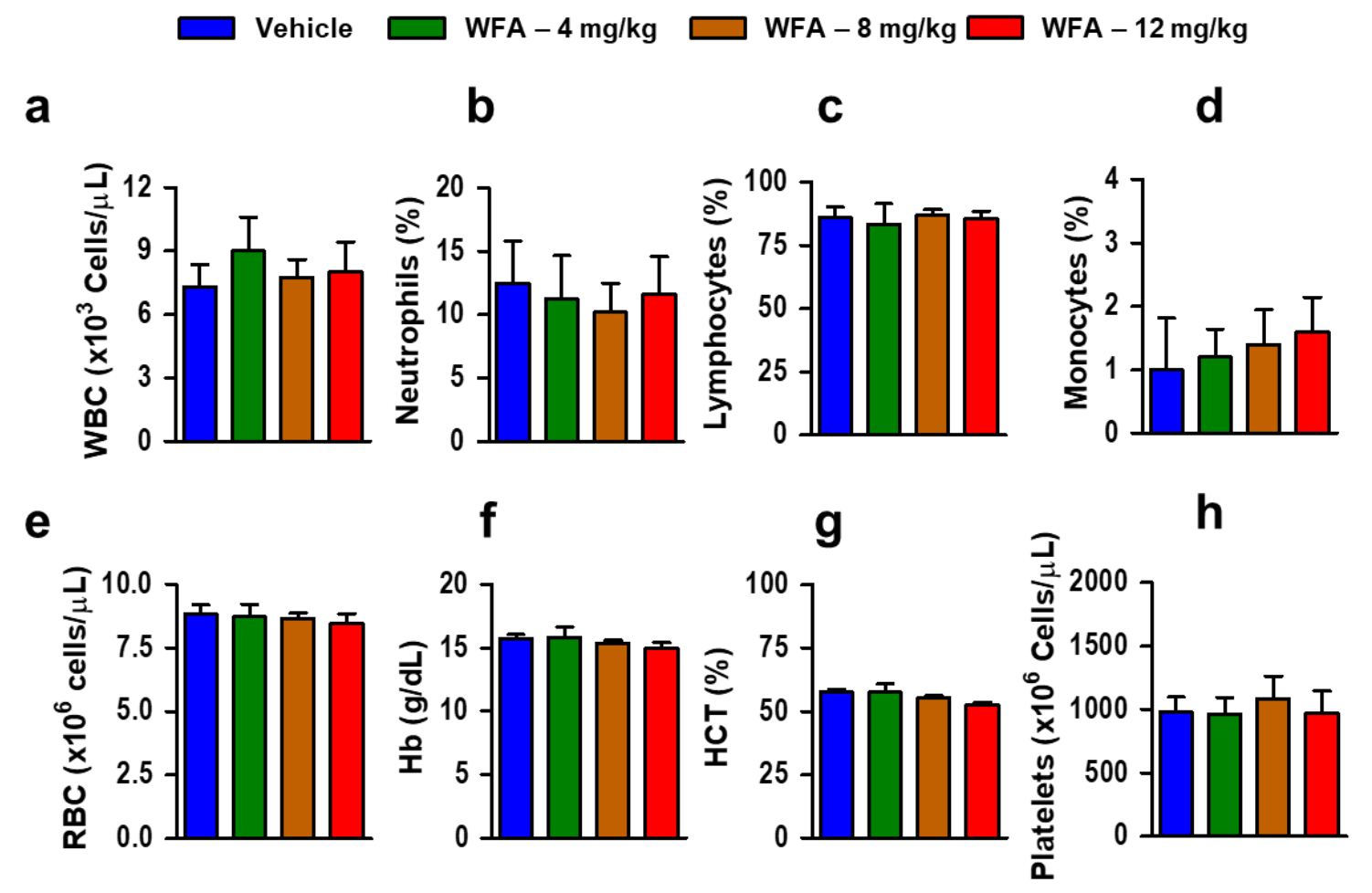

Fig 4.2. Effect of WFA on hematological parameters. After 28 days, rats were sacrificed under CO2 asphyxiation and terminal blood was collected by cardiac puncture. Mean; (a) Total white blood cell counts (WBC), (b) neutrophils, (c) lymphocytes, (d) monocytes, (e) total red blood cell counts (RBC), (f) hemoglobin (Hb), (g) hematocrit (HCT), and (h) total platelet counts. Data are presented as mean \pm SD for each group and were compared with the vehicle. No statistically significant changes in hematologic parameters were reported at all dose levels of WFA. 
a.
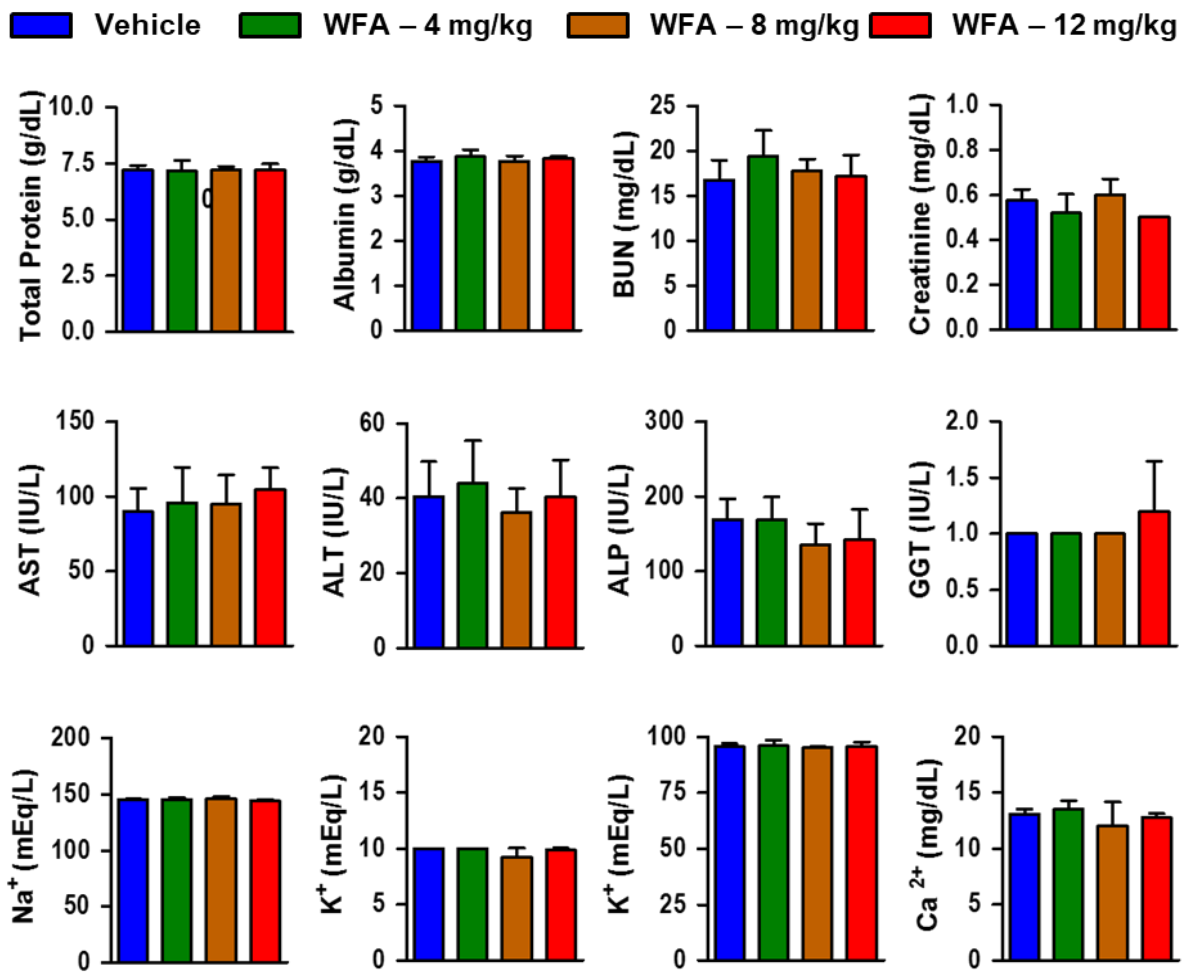

b.

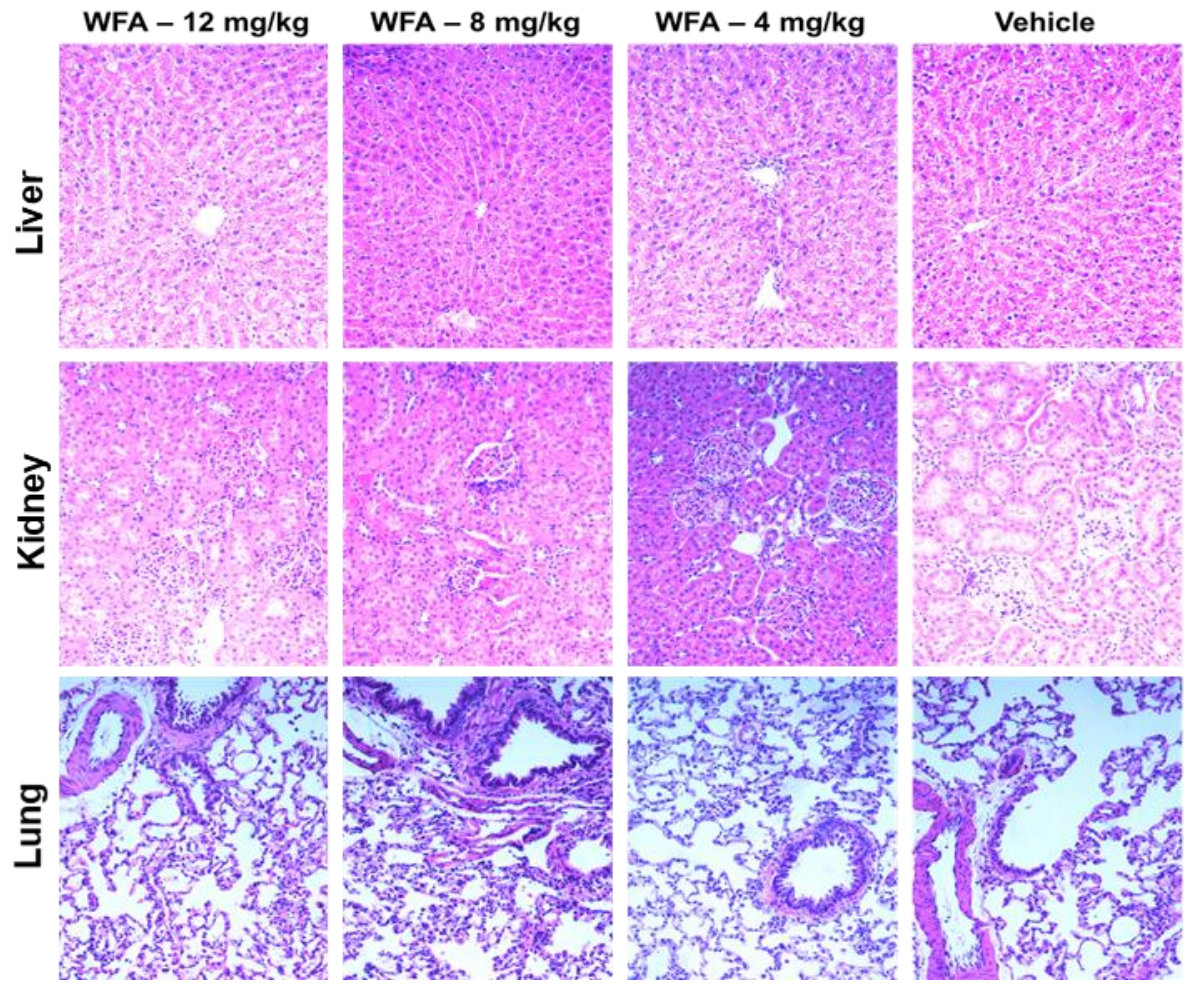

Fig 4.3. WFA did not cause biochemical or histological toxicity. (a) Serum was prepared and subjected to biochemical analysis for total protein, albumin, blood urea nitrogen (BUN), creatinine, AST, ALT, ALP, GGT, and electrolytes. (b) Representative photomicrographs of $\mathrm{H} \& \mathrm{E}$ stained tissue sections were prepared from organs collected at the termination of study. 


\section{HPLC-UV and HPLC-MS/MS analysis of WFA}

For the purposes of detection and quantification of WFA in plasma and tissues, we developed a highly sensitive and reproducible HPLC-MS/MS method. Previously, studies conducted in this laboratory [120] have used prednisolone as internal standard (IS) based on structural, chromatographic and extraction characteristics similarities with WFA. Similarly, in the present study, we used prednisolone as the internal standard. The HPLC chromatographic separation (1-10 $\mu$ L injections) and UV detection (Fig. 4.4a), showed that prednisolone $(\mathrm{RT}=5.7 \mathrm{~min})$ eluted before WFA $(\mathrm{RT}=6.3 \mathrm{~min})$. The maximum UV absorption for WFA was at $215 \mathrm{~nm}$ while the maximum absorbance for prednisolone was at $244 \mathrm{~nm}$. These two compounds, WFA (Fig. 4.4c) and prednisolone (Fig. 4.4d) displayed linear responses in the mean peak area versus concentration curves, and the peaks for both compounds were saturated at $100 \mu \mathrm{g} / \mathrm{mL}$. In addition, the peak area ratios (WFA/IS) were also plotted against WFA concentrations (Fig. 4.4e) and showed a linear relationship. The lower limit of detection (LLD) by HPLC- UV was $0.1 \mu \mathrm{g} / \mathrm{mL}$ for WFA and IS in methanol.

Since the main objective was to determine the levels of WFA in plasma and tissues, HPLC-UV was unsuitable for this analysis given the low UV absorbance of WFA (215 $\mathrm{nm})$. Therefore, we optimized a new analytical method in which we used HPLC separation coupled with MS/MS for detection and quantification of WFA levels. For both WFA and IS, we observed maximum responses in the positive MS/MS mode (Fig. 4.4b). Therefore, in positive MS/MS mode, we chose the m/z 471 and 361 as the precursor ions, 95 and 147 as the quantification ions for WFA and prednisolone, respectively. The m/z 281 and 299 ions were selected as qualifier ions for WFA while m/z 325 and 343 for prednisolone. The peak area ratios of WFA/IS versus concentration were plotted (Fig. 4.4f) 
a

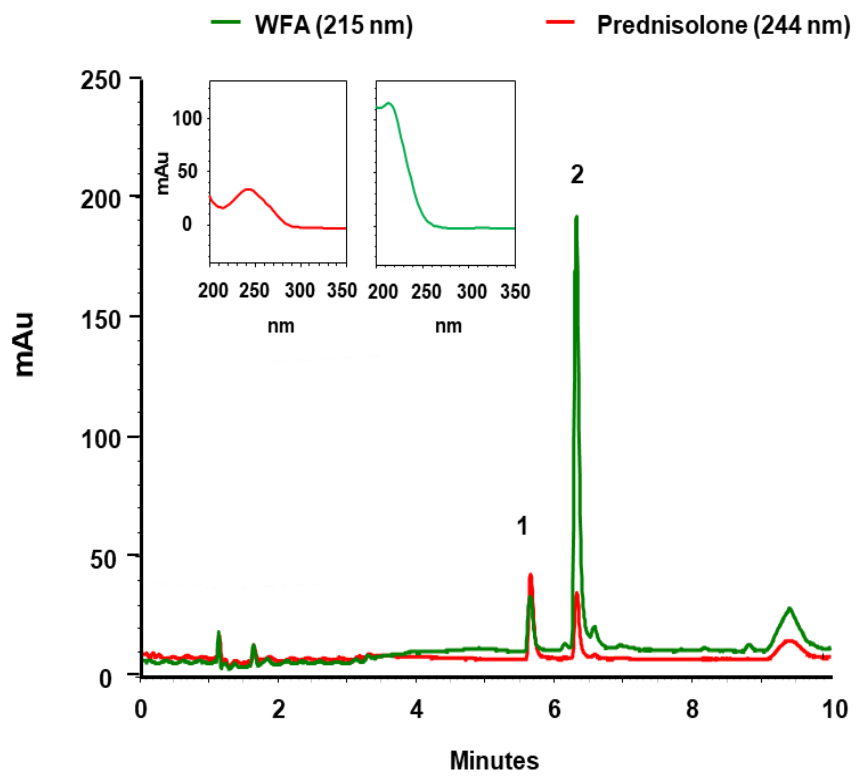

b
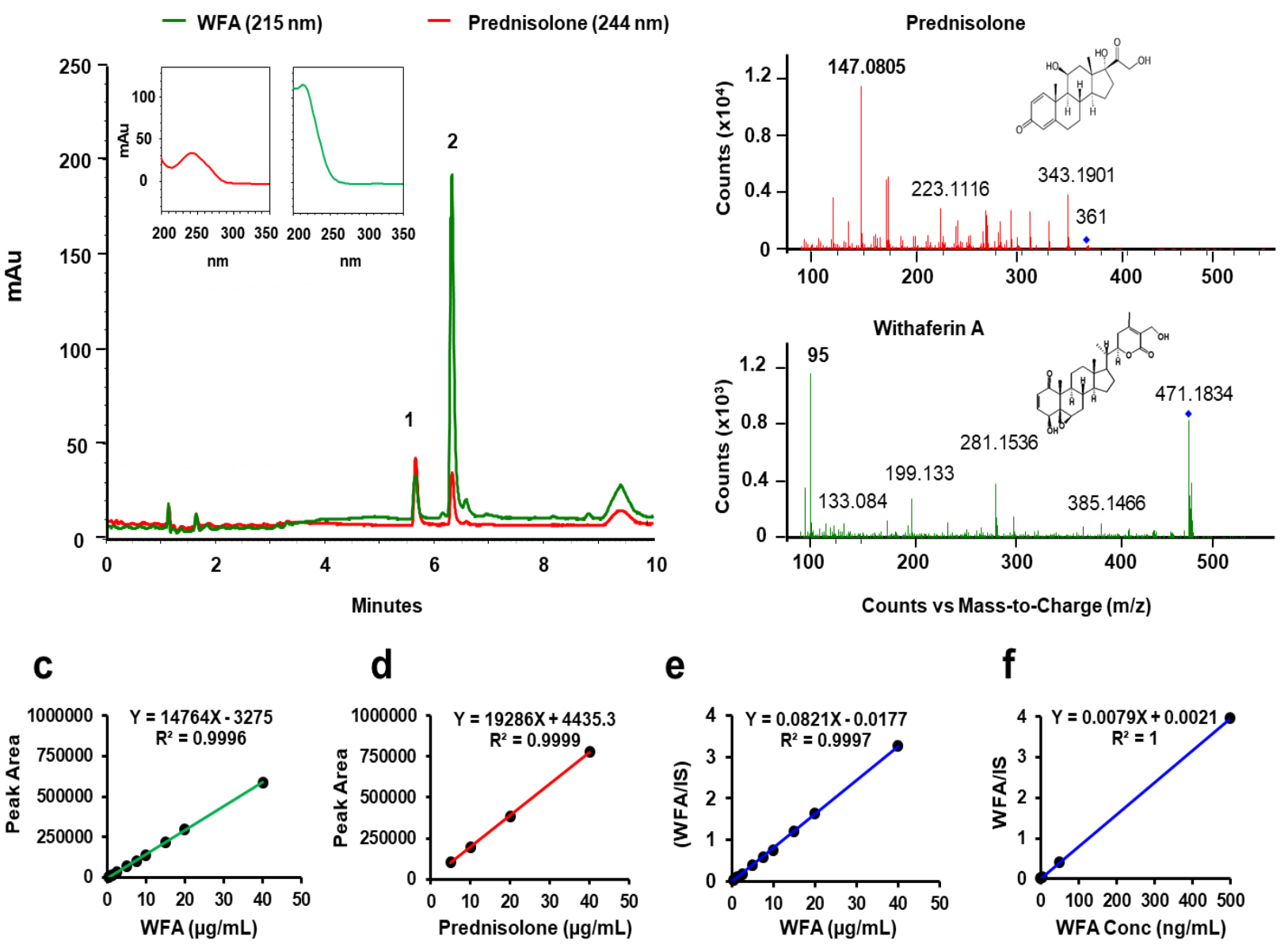

Figure 4.4. HPLC-MS/MS analysis of WFA. (a) Typical HPLC-UV chromatograpms obtained at $215 \mathrm{~nm}$ and $244 \mathrm{~nm}$ showing the peaks for (1) prednisolone (IS) in red and (2) WFA in green (volume of injection: $5 \mu \mathrm{L}$ ). (b) HPLC-MS/MS spectra for prednisolone and WFA. Standard curves based on peak areas were generated for (c) WFA, (d) prednisolone. (e) Using a $10 \mu \mathrm{g} / \mathrm{mL}$ IS, a standard curve based on peak area ratios (WFA/IS) was generated for WFA $(0-40 \mu \mathrm{g} / \mathrm{mL})$. (f) Standard curve for WFA using HPLC-MS/MS was generated for WFA $(0-500 \mathrm{ng} / \mathrm{mL})$. 


\section{Optimization of sample pre-treatment and method validation}

Several methods are available for the extraction of small molecules from biological matrices for quantitation purposes [121]. However, for each compound, there is need to optimize the method that results in the best extraction characteristics of the target analyte. In the present study, we developed and standardized a simple protein-precipitation method for the extraction of WFA from rat plasma and tissues. We found that for adequate and accurate quantitation, the sample preparation procedure required a matrix cleanup step to improve on the instrument sensitivity. We used $100 \mathrm{ng} / \mathrm{mL}$ prednisolone solution as the appropriate to monitor recoveries of WFA from plasma and tissue samples.

Initially, we investigated several crashing solvents to determine the best protein precipitation solvents. Notably, we compared acetonitrile (ACN) alone, acidified ACN (1 $\%$ formic acid), and a mixture of $\mathrm{ACN}$ and methanol $(\mathrm{MeOH})(1: 1)$. For all these solvent systems, we found out that there were no major differences in the recoveries of both WFA and the IS. However, using ACN alone provided the cleanest supernatant and had the least matrix interference in MS/MS (Fig. 4.5a). Therefore, we chose ACN as the extraction solvent to precipitate the proteins and disrupt the protein binding of WFA in plasma and tissue samples. It was determined that the best extract ratio of sample solution to ACN was 1:4 v/v. Next, when we compared the MS/MS responses of WFA in matrix versus without matrix and found that there was more than $70 \%$ ion suppression, particularly for the liver samples. This significantly decreased the sensitivity and accuracy of the instrument and the detection method. To decrease on the matrix interference, we used the Captiva EMR-Lipid solid-phase extraction (SPE) to preferentially remove phospholipids (Fig. 4.5b). As a result, Captiva EMR cartridges decreased the matrix effect from $>70 \%$ to $34 \%$. 


\section{Step 1:}

1. Weigh $1 \mathrm{~g}$ of tissue in $50 \mathrm{~mL}$ tube

2. Homogenize in $3 \mathrm{~mL} 0.9 \%$ saline solution

3. Add $100 \mu \mathrm{L}$ Internal Standard (IS) solution

\section{Step 2:}

1. Add $4 \mathrm{~mL}$ ice-cold $\mathrm{ACN}$ for $1 \mathrm{~mL}$ of homogenate

2. Vortex vigorously for $1 \mathrm{~min}$

3. Centrifuge at $8000 \mathrm{x}$ g for 20 minutes

4. Transfer the supernatant to a new tube

5. Dry under vacuum

\section{Step 3:}

1. Re-dissolve the residue $2 \mathrm{~mL}$ of $\mathrm{ACN} / \mathrm{H}_{2} \mathrm{O}(8: 2)$

2. Mix vigorously and centrifuge for $5 \mathrm{~min}$

3. Load on to $3 \mathrm{~mL}$ Captiva EMR-Lipid cartridge

4. Allow the sample to elute under gravity

5. Apply vacuum to dry the cartridge

6. Dry the supernatant under vacuum

Step 4:

1. Re-constitute in $100 \mu \mathrm{L}$ of $\mathrm{MeOH}$

2. The samples are ready for LCMS/MS analysis

Fig 4.5. Tissue and Plasma sample preparation scheme for WFA analysis by HPLC-MS/MS. 
a

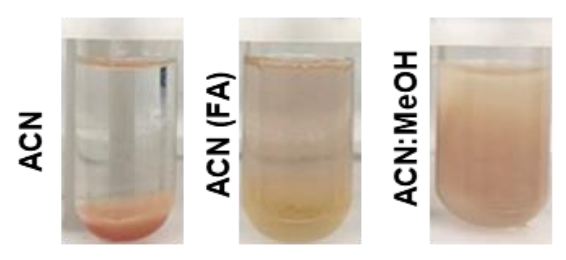

\section{Captiva EMR-Lipid Catridge}

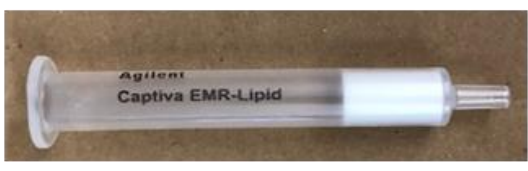

C

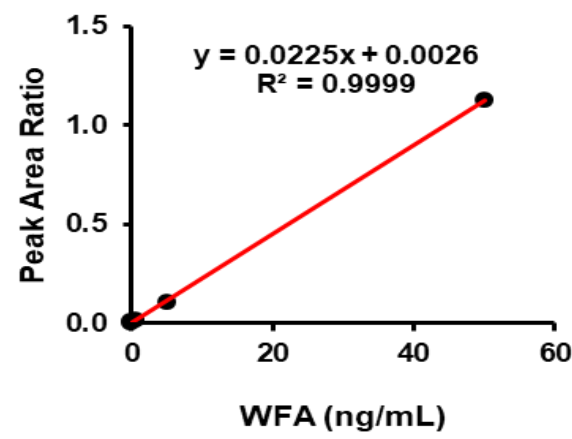

e

\section{Lung}

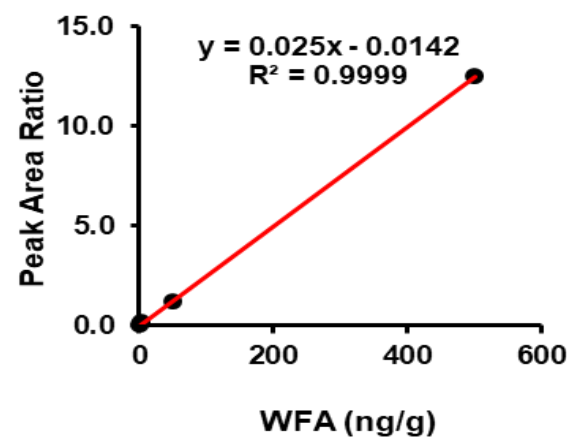

b

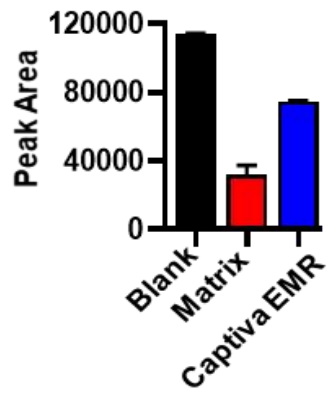

\begin{tabular}{|c|c|}
\hline Matrix & $\%$ Matrix Effect \\
\hline Liquid-Liquid & 72.2 \\
\hline Captiva-EMR Lipid & 34.4 \\
\hline
\end{tabular}

Kidney

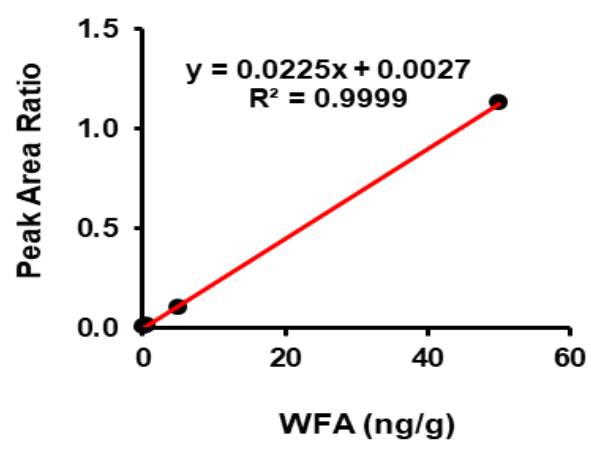

$\mathbf{f}$

Liver

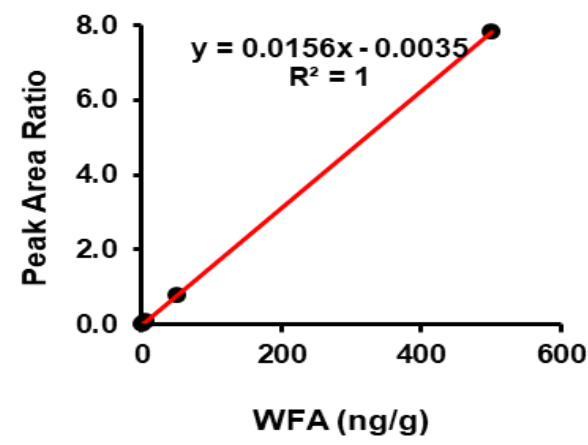

Fig 4.6. Sample matrix cleanup using Captiva-EMR Lipid SPE (a) Representative images showing protein-precipitation using solvents (acetonitrile: ACN, acetonitrile $+1 \%$ formic acid; $\mathrm{ACN}+1 \% \mathrm{FA}$, acetonitrile+methanol: $\mathrm{CAN}+\mathrm{MeOH}(1: 1)$. (b) Matrix clean-up was done using Captiva-EMR lipid SPE cartridges. Standard curves for WFA spiked in (c) plasma, (d) kidney, (e) lung, and (f) liver matrices. 


\section{Metabolic stability of WFA in SD rat liver microsomes}

In this study, the in vitro stability of WFA in microsomes was evaluated using SD rat microsomes at $37^{\circ} \mathrm{C}$. Briefly, $1 \mu \mathrm{M}$ WFA was incubated with SD rat liver microsomes $(0.1 \mathrm{mg}$ total protein) in a $200 \mu \mathrm{L}$ total reaction volume. A plot of peak area ratio (WFA/IS) indicated a rapid time-dependent disappearance of WFA in the reaction mixture (Fig 4.7a). The log-transformed data (Fig. 4.7b) showed a linear relationship between the peak area ratios and time with a slope of -0.044 . The elimination rate constant $(\mathrm{k}=-\mathrm{slope})$ of WFA in rat microsomes was calculated as 0.044 and the half-life was $16 \mathrm{~min}$. The intrinsic clearance of WFA in SD rat liver microsomes was $88 \mu \mathrm{L} / \mathrm{min} / \mathrm{mg}$ (Fig. 4.7c).

\section{The unbound fraction of WFA in SD rat plasma}

To determine the extent of plasma protein binding, known amounts of WFA were spiked in freshly collected SD rat plasma and the unbound drug was separated from the bound drug by an ultrafiltration method. Initially, we determined the non-specific binding (NSB) of WFA to the filter membrane by dissolving WFA in PBS $0.5 \mu \mathrm{g} / \mathrm{mL}$ (5\% DMSO). After centrifuging, the NSB of WFA to the Centrifree Ultrafiltration ${ }^{\circledR}$ device membrane was $<3 \%$ (Fig. 4.7d). By comparing the concentration of WFA in plasma prior to centrifugation (total), retentate and the filtrate, the fraction unbound $\left(f_{u}\right)$ of WFA was approximately 5\% (Fig. 4.7e). 

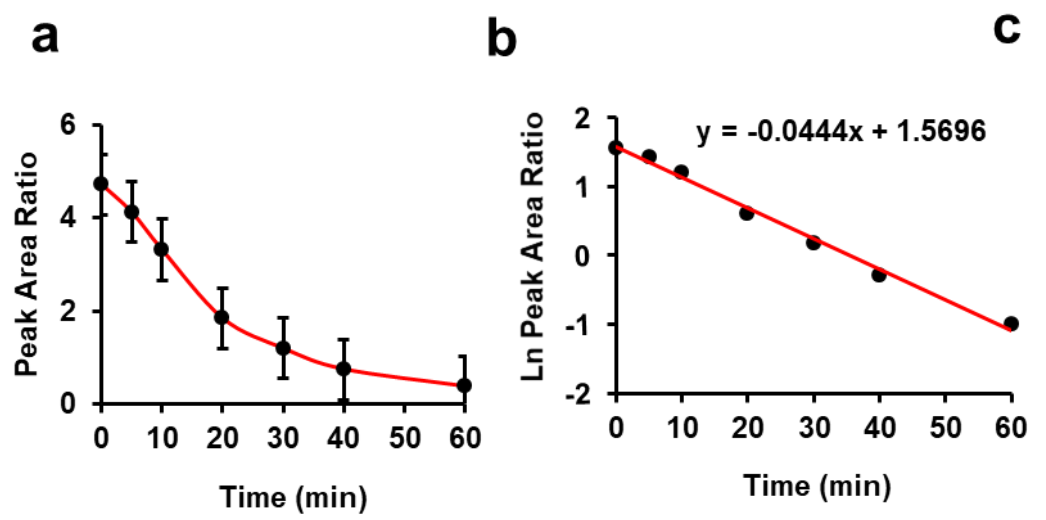

C
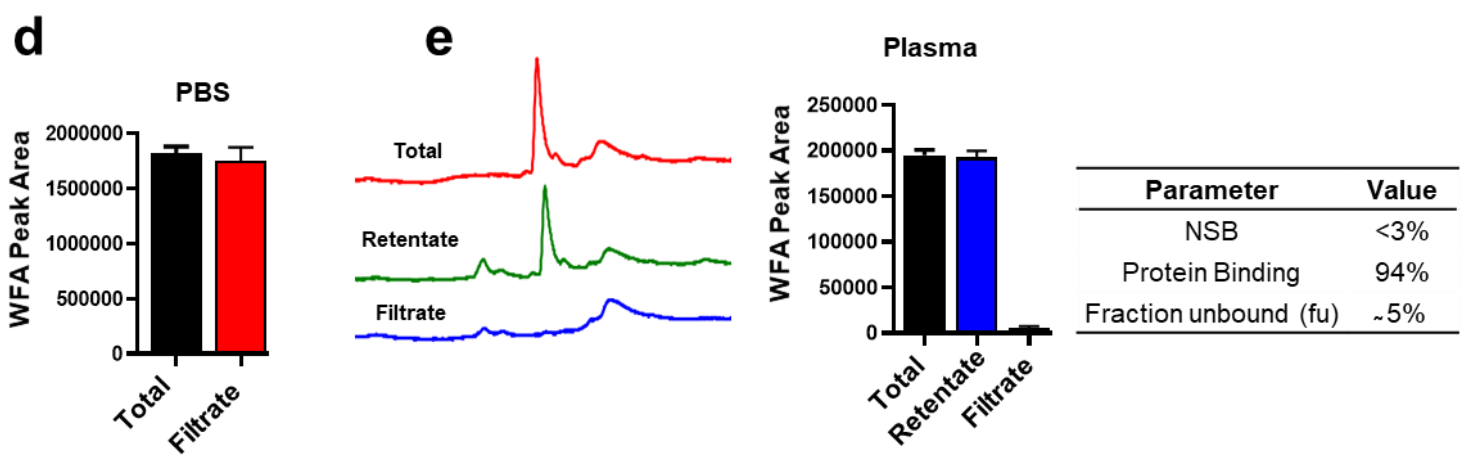

Fig 4.7. In vitro stability of WFA in liver microsomes and plasma protein binding. WFA (1 $\mu \mathrm{M})$ was incubated with SD rat microsomes and the fraction of parent compound remaining was determined by HPLC-MS/MS. The peak area ratios (WFA/IS) were plotted against time on (a) linear and (b) logarithmic scales. (c) The half-life $\left(\mathrm{t}_{1 / 2}\right)$ was $16 \mathrm{~min}$ and the in vitro intrinsic clearance $\left(\mathrm{Cl}_{\text {int }}\right)$ was $88 \mu \mathrm{L} / \mathrm{min} / \mathrm{mg}$. Plasma protein binding in fresh SD rat plasma was determined by ultrafiltration method. (d) Non-specific binding (NSB) was $<3 \%$. (e) Fraction unbound (fu) was calculated from the amount of WFA in filtrate versus total WFA in precentrifuged samples as approximately $5 \%$. 


\section{Pharmacokinetics of a single intravenous bolus dose of $10 \mathrm{mg} / \mathrm{kg}$ WFA in SD rats}

The pharmacokinetic properties of WFA were determined in male SD rats following the administration of a single intravenous bolus dose of $10 \mathrm{mg} / \mathrm{kg}$ WFA via the lateral vein. The concentrations of WFA in freshly collected plasma were determined by HPLC-MS/MS method as described in the methods section. The concentration-time plots of WFa in plasma (Fig 4.8a) demonstrated a rapid decline in the concentration of WFA with time. The mean concentrations of WFA in plasma rapidly fell below the limit of quantification within $2 \mathrm{~h}$ after dosing. The log-transformed concentration data (Fig. 4.8b) showed a linear relationship with time. The pharmacokinetic parameters of WFA were estimated using Phoenix WinNonLin 8.1 (Fig. 4.8c). The maximum plasma concentration was $907 \mathrm{ng} / \mathrm{mL}$ and was observed at the first sampling time point of 5 min after dosing. The last measurable concentration, $\mathrm{C}_{\text {last }}$, was $14 \mathrm{ng} / \mathrm{mL}$ at $2 \mathrm{~h}$, with an $\mathrm{AUC}_{\text {last }}$ of 15960 ng/mL*min and the $\mathrm{AUC}_{0 \text {-inf }}$ was $16709 \mathrm{ng}$ half-life $\left(\mathrm{t}_{1 / 2}\right)$ of WFA was 37 mins. The estimated terminal plasma clearance was $598 \mathrm{ml} / \mathrm{min} / \mathrm{kg}$. 

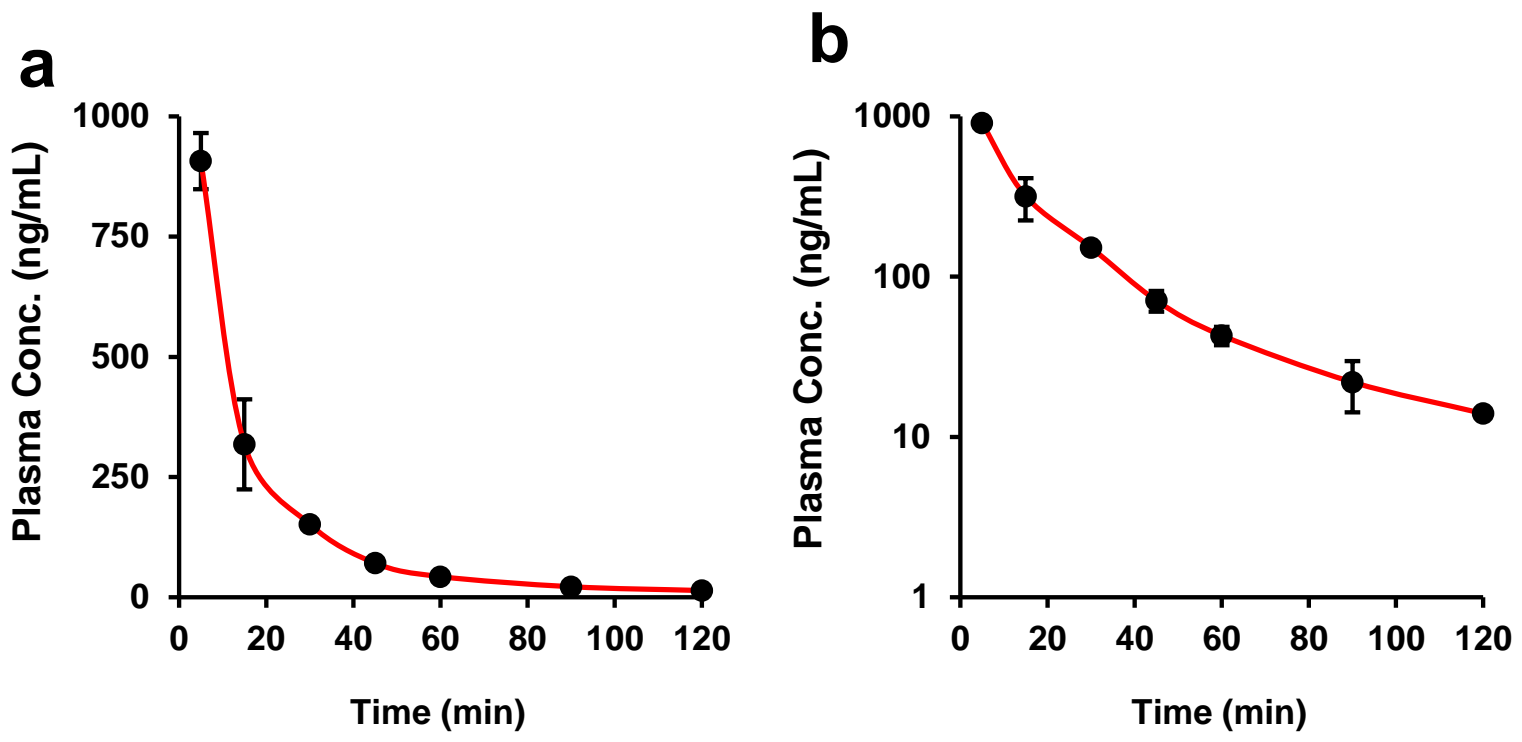

\begin{tabular}{cc}
\hline Pharmacokinetic Parameter & Value \\
\hline $\mathrm{Cmax}(\mathrm{ng} / \mathrm{mL})$ & 907 \\
$\mathrm{~T}_{\max }(\mathrm{min})$ & 5 \\
$\mathrm{C}_{\text {last }}(\mathrm{ng} / \mathrm{mL})$ & 14 \\
$\mathrm{AUC}_{0-\mathrm{t}}(\mathrm{ng} / \mathrm{mL} * \min )$ & 15960 \\
$\mathrm{AUC}_{0 \text {-inf }}(\mathrm{ng} / \mathrm{mL} * \min )$ & 16708 \\
$\mathrm{Extrapolation}(\%)$ & 4.3 \\
$\mathrm{t}_{1 / 2}(\mathrm{~min})$ & 37 \\
$\mathrm{CL}(\mathrm{mL} / \mathrm{min} / \mathrm{kg})$ & 598 \\
$\mathrm{AUMC}_{0-\mathrm{t}}(\mathrm{ng} / \mathrm{mL} * \min * \min )$ & 352613 \\
$\mathrm{AUMC}_{0 \text {-inf }}(\mathrm{ng} / \mathrm{mL} * \min * \min )$ & 482466 \\
\hline
\end{tabular}

Fig 4.8. Plasma pharmacokinetics of WFA in male SD rats. Plasma concentration $(\mathrm{ng} / \mathrm{mL})$ versus time curve profile of WFA following a single bolus intravenous injection of $10 \mathrm{mg} / \mathrm{kg}$ WFA in male SD rats. (a) Linear, and (b) Log-linear concentration versus time curves. (c) Noncompartmental analysis was perfomed using phoenix WinNonLin 8.1 to determine pharmacokinetic parameters. 


\section{DISCUSSION}

Many of the cytotoxic drugs used in the systemic chemotherapy of NSCLC require high doses to achieve clinically efficacious concentrations [13]. Traditionally, the dosage calculations for anticancer drugs are based on body surface area (BSA) which is derived from the body weight and height of patients [113]. However, this approach has proven inadequate and fallacious since several studies have shown that factors such as protein binding, metabolism, and clearance of drugs significantly affect the clinical efficacy of cancer treatment [112]. Moreover, cancer patient populations are often highly complex and varied in terms of age, presence of comorbidities or organ impairments [3]. In the current era of personalized medicine [12], dosing of patients with such highly toxic agents requires precise knowledge on the disposition characteristics of these agents. Today, the treatment guidelines for NSCLC involve multi-drug combinations which are administered via complex dosing schedules and sequences. Since there are limitations on the conduct of pharmacokinetic studies in cancer patients, the pharmacokinetic properties of most anticancer drugs used currently are poorly characterized. Therefore, to better predict the clinical efficacy of future cancer treatments, the potential anticancer agents must be well characterized in the preclinical setting for their toxicity and pharmacokinetic properties before studies are initiated in humans $[9,16]$.

Several published studies have demonstrated the potential therapeutic efficacy of WFA against multiple cancer types [22]. However, there is still very limited data describing the toxicity and pharmacokinetic properties of WFA to enable the design of appropriate doses and dosing schedules for clinical studies. As such, for several decades, the anticancer testing for the therapeutic efficacy of WFA is limited to preclinical studies. In addition, 
another major challenge to the study of the pharmacokinetics of WFA has been the lack of reliable, accurate and reproducible analytical methods for the detection and quantification of WFA in biological matrices. To address these challenges, we investigated the potential repeated dosing toxicity and the single-dose preclinical pharmacokinetic profile of WFA in male SD rats. Analytically, we have developed a highly sensitive HPLC-MS/MS method for the detection and quantification of WFA in plasma and tissues. In our studies, we have successfully applied this method to study the pharmacokinetics of WFA in males SD rats.

Ealier studies investigating the toxicity of WFA in experimental animals have been based on observation of animals dosed with standardized extracts of W. somnifera. Notably, studies have shown that alcoholic extracts of W. somnifera extract (up to 2000 $\mathrm{mg} / \mathrm{kg}$ ) were non-toxic to Wistar rats after 14-days repeated oral dosing [122]. Similar studies have reported no signs of systemic or histological toxicity following repeated subchronic dosing of WFA in mice $[123,124]$. Although it is evident that extracts containing WFA have been evaluated for their toxicity, the extracts used in these studies contained $<5 \%$ total WFA (\% w/w). Contrastingly, we have investigated the potential toxicity of pure WFA (>95\% purity) in male SD rats. In agreement with previously published studies, the findings presented here show that orally administered WFA is essentially non-toxic to male SD rats. While we observed a slight dose-dependent increase in body weight in the groups of rats receiving WFA, these changes were nonsignificant. Moreover, evaluations of the average food intake indicated that the observed body weight gains were correlated with higher food intake. More importantly, systemic data did not reveal any evidence of bone marrow suppression since parameters such as total RBC, WBC, $\mathrm{Hb}$, and HCT were all 
comparable to vehicle-treated rats. Therefore, the doses $(4,8$, and $12 \mathrm{mg} / \mathrm{kg})$ of WFA that we used in the present study were generally well tolerated and safe in male rats.

One major hindrance to the study of the pharmacokinetics of WFA has been the lack of appropriate analytical methods. In literature, there are a few HPLC and HPLCESI-MS/MS methods for the detection of have been published [125-127]. It has been reported that these methods could detect and quantify WFA in plasma up to a lower limit of $50 \mathrm{ng} / \mathrm{mL}$. Unfortunately, we failed to reproduce these methods as many did not include sufficient experimental details. Whereas there are methods for detection of WFA in plasma, limited information is available for the detection of WFA levels in tissue organs such as liver, lungs, and kidneys. Moreover, during our preliminary studies, we compared the effect of the matrix from plasma to that of other tissues and found that tissue matrices, especially from the liver significantly suppressed ionization and subsequent detection of WFA by LCMS/MS conditions. Therefore, while the authors [125] argue that the published methods could successfully be applied to quantify WFA in plasma, the methods were not applicable to tissues. Recently, studies done and published from this laboratory reported a liquidliquid extraction method for the detection and quantification of WFA in tissues. Along the same lines, we have made improvements to this method by adding a solid phase matrix clean-up step instead of liquid-liquid sample preparation.

During our preliminary sample preparation studies, we investigated multiple protein precipitation solvents to determine the most suitable of WFA. Though sample preparation was adequate for both methanol and acetonitrile, the supernatants from samples prepared with methanol were found to be turbid and the protein pellets were quite unstable. Therefore, sample preparation with methanol (100\% or $50 \%)$ was deemed unsuitable for 
the extraction since it produced significant matrix interference with the analytical conditions. On the other hand, acetonitrile produced much better response and was selected as the crushing solvent for sample preparation in subsequent studies. Further, in our analysis, whereas protein precipitation with acetonitrile eliminated most proteins, there was still significant interference on the ionization of WFA from lipids and phospholipids in the extracted samples. To address this challenge, we examined several SPE methods for matrix removal and found that the Captiva EMR-lipid cartridges (Agilent Technologies), which are specially made for the removal of phospholipids. Intriguingly, this step significantly improved the sensitivity of LC-MS/MS detection conditions and by applying a 10-fold concentration of the original samples (by reconstituting samples $(1 \mathrm{~g}$ tissue or $1 \mathrm{~mL}$ plasma) in to $100 \mu \mathrm{L}$ final volume, we improved the detection of WFA to up to $5 \mathrm{ng} / \mathrm{g}$ of tissue or $1 \mathrm{ng} / \mathrm{ml}$ of plasma. Our recovery of both WFA and internal standard was greatest from plasma samples (>80\%) and lowest in liver samples (approx. 75\%).

After developing the analytical method, we next investigated the pharmacokinetic properties of WFA in SD rats. Previously, studies conducted in mice have investigated the pharmacokinetics of WFA $[31,128]$. However, whereas plasma pharmacokinetics were investigated after oral administration in these studies, no data on protein binding capacity or metabolic stability in microsomes are reported. Therefore, in our studies, we investigated both the plasma protein binding capacity and metabolic stability of WFA in liver microsomes in SD rats. Our data show that WFA displays a high protein binding capacity in rat plasma resulting in a with a very low fraction unbound (fu). Since pharmacokinetic properties such as hepatic metabolism, clearance, and volume of distribution are heavily dependent on to the unbound fraction of drugs there is a significant potential of WFA to 
interact with drugs that show similar protein binding characteristics. In addition, WFA displayed a high intrinsic hepatic clearance ( $>80 \mu \mathrm{l} / \mathrm{min} / \mathrm{mg}$ ) indicating that the liver plays a critical role in the clearance of WFA from the body. Further, based on our plasma concentration-time data, we show that WFA has linear pharmacokinetics in SD rats. Following intravenous bolus administration, there was very rapid distribution and clearance of WFA from plasma. There was a high volume of distribution greater than the total body water indicating the extensive distribution of WFA in tissues. Since concentrations of WFA fell below the limit of detection in $2 \mathrm{~h}$, it appears slow intravenous infusions would be the best mode administration. The observed short terminal plasma $\mathrm{t}_{1 / 2}$ was in tandem with the observed rapid intrinsic clearance of WFA in rat microsomes indicating the potential role of the liver in the clearance of WFA. Since urine was never collected in the present studies, we did not determine the renal clearance of WFA. Nonetheless, our observed plasma pharmacokinetic parameters were similar to those obtained in mice after intraperitoneal and oral dosing of WFA.

In conclusion, WFA is a promising antitumor agent for the treatment of multiple types of cancers. In the present study, we have investigated the potential oral toxicity and preclinical pharmacokinetic properties of WFA SD rats. We conclude that oral dosing of up $12 \mathrm{mg} / \mathrm{kg}$ of WFA was safe and well-tolerated without any signs of gross or systemic toxicity in male SD rats. Further, WFA displays a high protein binding capacity to plasma proteins indicating a potential for drug-drug interactions. Moreover, WFA displayed rapid clearance in liver microsomes and plasma indicating that slow continuous infusion would be the most appropriate mode of administration. Further studies to determine the tissuedistribution of WFA, particularly in the tumor-bearing animals are highly recommended. 


\section{CHAPTER FIVE}

\section{OVERALL DISCUSSION, SUMMARY, AND CONCLUSIONS}

Lung cancer remains the leading cause of cancer-related deaths among both men and women worldwide, and in the United States. NSCLC contributes to 85-90\% of all lung cancer cases and has an overall survival rate of $15 \%$ after five years. The major reason for the dismal prognosis of NSCLC is that $60-70 \%$ of patients are diagnosed when the disease is at an advanced stage. As such, the tumors in many NSCLC patients are not amenable to curative surgery and most of the patients succumb in less than one year after diagnosis. Thus, systemic chemotherapy remains the only viable treatment option in the neoadjuvant, adjuvant or palliative setting for advanced NSCLC.

Recently, there has been a paradigm shift in the treatment approaches for advanced NSCLC. This was mainly prompted by the landmark discoveries of molecularly targeted drugs and immunotherapies for NSCLC. However, despite these advancements, only marginal improvements in progression-free survival but not overall survival have been reported. Besides the high cost of therapy, targeted and immunotherapies only benefit select groups of patients that fit a strict inclusion. Therefore, for the majority of NSCLC patients, systemic treatment involves the administration of multiple cycles of high doses of cytotoxic agents. Currently, cisplatin (cis-Pt) alongside paclitaxel (PAC) is the front-line treatment regimen in the clinic for stage IIIB-V metastatic NSCLC [11, 129]. However, chemoresistance, metastasis, and dose-limiting toxicities limit the therapeutic efficacy to a 
maximum of 14 months. In this doctoral thesis, we leverage the ability of WFA, a highly efficacious plant-derived steroidal lactone to enhance the chemotherapeutic efficacy of PAC against NSCLC. The main hypothesis was that WFA alongside PAC is a highly synergistic approach, which shows greater antitumor efficacy than the current platinumbased chemotherapies. To test this hypothesis, we designed experiments to; (1) explore the synergistic combination of PAC and WFA against human NSCLC cells, (2) determine the effect of WFA on epithelial to mesenchymal transition (EMT), (3) determine the safety and pharmacokinetic properties of WFA in experimental animals.

To demonstrate the in vitro antitumor efficacy, we used two human NSCLC cell lines, H1299 (large cell carcinoma) and A549 (adenocarcinoma). Firstly, we showed that that WFA alone was active against the growth and proliferation of both H1299 and A549 cell lines. The effects of WFA were both doses- and time-dependent, with median inhibitory concentrations in the sub-micromolar range. As reported previously [130, 131], WFA increased the production of reactive oxygen species (ROS) and induced cellular apoptosis in NSCLC cells. The cytotoxic effects of WFA blocked the thiol donors dithiothreitol (DTT) and N-acetyl cysteine (NAC). Therefore, we conclude and provide evidence for the hypothesis that WFA induced apoptosis in NSCLC via thiol-dependent mechanisms [130]. Next, we performed multiple drug combination studies consisting of two drugs of either PAC, cis-Pt or WFA to determine the most efficacious combination against cellular proliferation. Our data show that the combination of PAC+WFA was more efficacious than PAC+cisPt, or cisPt+WFA. There was a dramatic increase in the potency and efficacy of PAC when combined with WFA at 1:40, 1:20 and 1:10 combinatorial ratios, respectively. Importantly, pretreatment of cells with WFA increased the synergistic effects 
of PAC and WFA indicating a chemosensitizing effect of WFA on NSCLC cells to standard chemotherapeutic drugs. Together, the data presented demonstrate that the use of WFA alongside PAC is a very attractive and viable alternative therapeutic strategy, and provides a strong rationale for further testing of this combination in animal models and clinical studies.

Today, $>90 \%$ of deaths in NSCLC are directly attributable to chemoresistance and metastasis [13]. This prompted us the investigate whether WFA alone or in combination with PAC targets chemoresistance in NSCLC. First, we used both drug-sensitive and drugresistant cells to determine the effects on cellular proliferation and mechanisms of drug resistance. Interestingly, our data show that PAC-resistant (TR-A549) cells were crossresistant to cis-Pt but not to WFA. These cells overexpressed the MDR1 protein, an indicator that increased drug efflux was the main mechanism of resistance [73]. Further, PDL-1 expression was upregulated while E-cadherin was downregulation suggesting a strong link between EMT, drug resistance and immune evasion in NSCLC. Thus, it appears that the resistance of TR-A549 cells to PAC was associated with an upregulation of drug efflux mechanisms, immune evasion, and EMT. When xenografted in athymic nude mice, WFA significantly inhibited tumor growth PAC-resistant cells. Moreover, WFA treated downregulated the expression of MDR1 in PAC resistant cells. Mechanistically, WFA inhibited EMT induction via the regulation of smad $2 / 3$ and NF- $\kappa \mathrm{B}$ signaling in a dose- and time-dependent manner. In summary, these findings strongly suggest that WFA is highly active against both drug-sensitive and drug-resistant NSCLC cells and its ability to inhibit EMT in NSCLC cells demonstrates the potential of WFA to prevent or delay the emergence of chemoresistance and metastasis in NSCLC during treatment. 
Also, while multiple studies have reported the therapeutic efficacy of WFA, there is very limited data on its potential toxicity and pharmacokinetics. In this thesis, we examined the toxicity of WFA in male SD rats. Previously, some studies [122] have indirectly investigated the toxicity of WFA in mice and rats by administering standardized alcoholic extracts of W. somnifera. However, the limitation was that the content of WFA in these extracts was very low $(<1 \% \mathrm{w} / \mathrm{w})$, and considering the chemical complexity of $W$. somnifera extracts, it is not possible to delineate the effects of WFA from other compounds. In contrast, we have evaluated the potential toxicity of repeated oral dosing of highly pure WFA (>95\%, HPLC) and found that WFA did not cause any signs of toxicity in a 28 -day study. In our study, up to $12 \mathrm{mg} / \mathrm{kg}$ of WFA was given as three doses per week via oral gavage and did not cause any changes in hematological, biochemical or histopathological parameters. Next, a highly sensitive, reproducible and robust HPLC-MS/MS method for the detection and quantification of WFA in plasma and tissue samples was developed. In this method, WFA from plasma or tissues is extracted by simple liquid-liquid extraction followed an SPE matrix cleanup step using Captiva-EMR lipid removal cartridges. While other methods have been previously published [125], all these were not suitable for analysis of WFA in tissue by LC-MS/MS due to significant ion suppression. Using our method, the sensitivity of LC-MS/MS was increased up to $80 \%$ for the detection of WFA in tissue samples.

To study the pharmacokinetic properties of WFA, we determined the plasma protein binding capacity, in vitro clearance in microsomes, and the plasma pharmacokinetic properties of WFA in male SD rats. Using in vitro methods, we demonstrate that WFA displays a high plasma protein binding (>95\%) capacity as measured by ultrafiltration. 
Interestingly, when incubated in liver microsomes, there was a high intrinsic hepatic clearance of WFA $\left(\mathrm{Cl}_{\text {int }}: 88 \mu \mathrm{L} / \mathrm{min} / \mathrm{mg}\right)$. In male SD rats, WFA displayed an instant distribution phase and rapid clearance from plasma. Following a single bolus dose of 10 $\mathrm{mg} / \mathrm{kg}$, the levels of WFA were below the limit of detection within $2 \mathrm{~h}$. Together, these findings suggest that WFA has a high volume of distribution in tissues, a high protein binding capability to plasma proteins and hepatic clearance is the main elimination route for WFA. Overall, our studies demonstrate that WFA is well tolerated and safe following repeated oral dosing in rats. We developed a detailed method for the determination of WFA in biological matrices and have used this method to study the pharmacokinetic properties of WFA in SD rats.

In future studies, it is important that the synergistic effects of PAC and WFA are validated in animal studies before clinical studies can be initiated. It is well known that PAC treatment induces chemoresistance and metastasis mechanisms in NSCLC cells. Therefore, we recommend that studies be conducted to investigate whether WFA when used alongside PAC, can prevent the emergence of chemoresistance and metastasis in NSCLC. Furthermore, both PAC and WFA show high protein binding and clearance in liver microsomes. Therefore, it is important to study the pharmacokinetics of PAC+WFA to identify potential pharmacokinetic drug interactions. 


\section{REFERENCES}

1. Torre, L.A., et al., Global Cancer Incidence and Mortality Rates and Trends-An Update. Cancer Epidemiology Biomarkers \&amp; Prevention, 2016. 25(1): p. 1627.

2. Bray, F., et al., Global cancer statistics 2018: GLOBOCAN estimates of incidence and mortality worldwide for 36 cancers in 185 countries. CA Cancer J Clin, 2018. 68(6): p. 394-424.

3. Siegel, R.L., K.D. Miller, and A. Jemal, Cancer statistics, 2019. CA: A Cancer Journal for Clinicians, 2019. 69(1): p. 7-34.

4. Barta, J.A., C.A. Powell, and J.P. Wisnivesky, Global Epidemiology of Lung Cancer. Ann Glob Health, 2019. 85(1).

5. Celik, B., T. Bulut, and A. Khoor, Subtyping of non-small cell lung cancer by cytology specimens: A proposal for resource-poor hospitals. Cytojournal, 2019. 16: p. 8.

6. Herbst, R.S., J.V. Heymach, and S.M. Lippman, Lung Cancer. New England Journal of Medicine, 2008. 359(13): p. 1367-1380.

7. Haura, E.B., et al., Molecular origins of lung cancer: prospects for personalized prevention and therapy. J Thorac Oncol, 2010. 5(6 Suppl 3): p. S207-13.

8. Liam, C.K., et al., Lung cancer staging now and in the future. Respirology, 2015. 20(4): p. 526-34.

9. Zhang, C., et al., Emerging therapies for non-small cell lung cancer. 2019. 12(1): p. 45.

10. McGuire, S., World Cancer Report 2014. Geneva, Switzerland: World Health Organization, International Agency for Research on Cancer, WHO Press, 2015. Adv Nutr, 2016. 7(2): p. 418-9.

11. Lemjabbar-Alaoui, H., et al., Lung cancer: Biology and treatment options. Biochimica et biophysica acta, 2015. 1856(2): p. 189-210.

12. Politi, K. and R.S. Herbst, Lung cancer in the era of precision medicine. Clinical cancer research : an official journal of the American Association for Cancer Research, 2015. 21(10): p. 2213-2220.

13. De Marinis, F., et al., Diagnosis and first-line treatment of non-small cell lung cancer in the era of novel immunotherapy: recommendations for clinical practice. Expert Rev Respir Med, 2019. 13(3): p. 217-228.

14. Chen, R., et al., Comparative efficacy and safety of first-line treatments for advanced non-small cell lung cancer with immune checkpoint inhibitors: A systematic review and meta-analysis. Thorac Cancer, 2019. 10(4): p. 607-623.

15. Spira, A., B. Halmos, and C.A. Powell, Update in Lung Cancer 2014. Am J Respir Crit Care Med, 2015. 192(3): p. 283-94. 
16. Baxevanos, P. and G. Mountzios, Novel chemotherapy regimens for advanced lung cancer: have we reached a plateau? Annals of translational medicine, 2018. 6(8): p. 139-139.

17. Politi, K. and R.S. Herbst, Lung Cancer in the Era of Precision Medicine. Clinical Cancer Research, 2015. 21(10): p. 2213-2220.

18. ACS, Cancer facts\&figures 2015. Report No.: 500815. Available from: http://www.cancer.org/acs/groups/content/

@editorial/documents/document/acspc-044552.pdf. 2015.

19. Tong, X., H. Zhang, and B.N. Timmermann, Chlorinated Withanolides from Withania somnifera. Phytochem Lett, 2011. 4(4): p. 411-414.

20. Mirjalili, M.H., et al., Steroidal lactones from Withania somnifera, an ancient plant for novel medicine. Molecules, 2009. 14(7): p. 2373-93.

21. Samadi, A.K., Potential Anticancer Properties and Mechanisms of Action of Withanolides. Enzymes, 2015. 37: p. 73-94.

22. Lee, I.C. and B.Y. Choi, Withaferin-A-A Natural Anticancer Agent with Pleitropic Mechanisms of Action. Int J Mol Sci, 2016. 17(3).

23. Lavie, D., E. Glotter, and Y. Shvo, 1371. Constituents of Withania somnifera Dun. Part IV. The structure of withaferin A. Journal of the Chemical Society (Resumed), 1965(0): p. 7517-7531.

24. Devi, P.U., Withania somnifera Dunal (Ashwagandha): potential plant source of a promising drug for cancer chemotherapy and radiosensitization. Indian J Exp Biol, 1996. 34(10): p. 927-32.

25. Prakash, J., et al., Chemopreventive activity of Withania somnifera in experimentally induced fibrosarcoma tumours in Swiss albino mice. Phytother Res, 2001. 15(3): p. 240-4.

26. Fuska, J., et al., Novel cytotoxic and antitumor agents. IV. Withaferin A: relation of its structure to the in vitro cytotoxic effects on P388 cells. Neoplasma, 1984. 31(1): p. 31-6.

27. Bharitkar, Y.P., et al., Chemistry of withaferin-A: chemo, regio, and stereoselective synthesis of novel spiro-pyrrolizidino-oxindole adducts of withaferin-A via one-pot three-component [3+2] azomethine ylide cycloaddition and their cytotoxicity evaluation. Mol Divers, 2015. 19(2): p. 251-61.

28. Vanden Berghe, W., et al., Molecular insight in the multifunctional activities of Withaferin A. Biochem Pharmacol, 2012. 84(10): p. 1282-91.

29. Vyas, A.R. and S.V. Singh, Molecular targets and mechanisms of cancer prevention and treatment by withaferin a, a naturally occurring steroidal lactone. AAPS J, 2014. 16(1): p. 1-10.

30. Patel, K., R.B. Singh, and D.K. Patel, Pharmacological and analytical aspects of withaferin A: A concise report of current scientific literature. Asian Pacific Journal of Reproduction, 2013. 2(3): p. 238-243.

31. Thaiparambil, J.T., et al., Withaferin A inhibits breast cancer invasion and metastasis at sub-cytotoxic doses by inducing vimentin disassembly and serine 56 phosphorylation. Int J Cancer, 2011. 129(11): p. 2744-55.

32. Bharitkar, Y.P., et al., Chemistry of withaferin-A: chemo, regio, and stereoselective synthesis of novel spiro-pyrrolizidino-oxindole adducts of withaferin-A via one-pot 
three-component [3+2] azomethine ylide cycloaddition and their cytotoxicity evaluation. Molecular Diversity, 2015. 19(2): p. 251-261.

33. Misra, L., et al., Selective reactivity of 2-mercaptoethanol with 5beta,6beta-epoxide in steroids from Withania somnifera. Steroids, 2008. 73(3): p. 245-51.

34. Mohan, R., et al., Withaferin A is a potent inhibitor of angiogenesis. Angiogenesis, 2004. 7(2): p. 115-22.

35. Stan, S.D., et al., Withaferin A causes FOXO3a- and Bim-dependent apoptosis and inhibits growth of human breast cancer cells in vivo. Cancer Res, 2008. 68(18): p. 7661-9.

36. Hahm, E.R., et al., Metabolic alterations in mammary cancer prevention by withaferin A in a clinically relevant mouse model. J Natl Cancer Inst, 2013. 105(15): p. 1111-22.

37. Hahm, E.-R. and S.V. Singh, Withaferin A-induced apoptosis in human breast cancer cells is associated with suppression of inhibitor of apoptosis family protein expression. Cancer Letters, 2013. 334(1): p. 101-108.

38. Yang, H., G. Shi, and Q.P. Dou, The tumor proteasome is a primary target for the natural anticancer compound Withaferin A isolated from "Indian winter cherry". Mol Pharmacol, 2007. 71(2): p. 426-37.

39. Suman, S., et al., Oral administration of withaferin A inhibits carcinogenesis of prostate in TRAMP model. Oncotarget, 2016.

40. Munagala, R., et al., Bovine milk-derived exosomes for drug delivery. Cancer Lett, 2016. 371(1): p. 48-61.

41. Kakar, S.S., V.R. Jala, and M.Y. Fong, Synergistic cytotoxic action of cisplatin and withaferin A on ovarian cancer cell lines. Biochem Biophys Res Commun, 2012. 423(4): p. 819-25.

42. Kakar, S.S., et al., DOXIL when combined with Withaferin A (WFA) targets ALDH1 positive cancer stem cells in ovarian cancer. J Cancer Stem Cell Res, 2016. 4.

43. Kakar, S.S., et al., Withaferin a alone and in combination with cisplatin suppresses growth and metastasis of ovarian cancer by targeting putative cancer stem cells. PLoS One, 2014. 9(9): p. e107596.

44. Siegel, R.L., K.D. Miller, and A. Jemal, Cancer statistics, 2016. CA: A Cancer Journal for Clinicians, 2016. 66(1): p. 7-30.

45. Ruchalski, K.L. and K. Brown, Lung Cancer Screening Update. Journal of Thoracic Imaging, 2016. 31(4): p. 190-200.

46. Gadgeel, S.M., S.S. Ramalingam, and G.P. Kalemkerian, Treatment of lung cancer. Radiol Clin North Am, 2012. 50(5): p. 961-74.

47. Chang, A., Chemotherapy, chemoresistance and the changing treatment landscape for NSCLC. Lung Cancer, 2011. 71(1): p. 3-10.

48. Cufer, T. and L. Knez, Update on systemic therapy of advanced non-small-cell lung cancer. Expert Rev Anticancer Ther, 2014. 14(10): p. 1189-203.

49. Sangha, R., J. Price, and C.A. Butts, Adjuvant Therapy in Non-Small Cell Lung Cancer: Current and Future Directions. The Oncologist, 2010. 15(8): p. 862-872.

50. Ernani, V., C.E. Steuer, and M. Jahanzeb, The End of Nihilism: Systemic Therapy of Advanced Non-Small Cell Lung Cancer. Annu Rev Med, 2017. 68: p. 153-168.

51. Aggarwal, C., Targeted therapy for lung cancer: present and future. Annals of Palliative Medicine, 2014. 3(3): p. 229-235. 
52. Lukas, R.V., et al., Systemic therapies in the treatment of non-small-cell lung cancer brain metastases. Future Oncol, 2016. 12(8): p. 1045-58.

53. Doroshow, D.B., et al., Immunotherapy in Non-Small Cell Lung Cancer: Facts and Hopes. Clin Cancer Res, 2019.

54. Stinchcombe, T.E., Targeted Therapies for Lung Cancer. Cancer Treat Res, 2016. 170: p. 165-82.

55. Schrank, Z., et al., Current Molecular-Targeted Therapies in NSCLC and Their Mechanism of Resistance. Cancers, 2018. 10(7): p. 224.

56. Ran, X. and K. Yang, Inhibitors of the PD-1/PD-L1 axis for the treatment of head and neck cancer: current status and future perspectives. Drug Des Devel Ther, 2017. 11: p. 2007-2014.

57. Rossi, A. and M. Di Maio, Platinum-based chemotherapy in advanced non-smallcell lung cancer: optimal number of treatment cycles. Expert Rev Anticancer Ther, 2016. 16(6): p. 653-60.

58. Dasari, S. and P.B. Tchounwou, Cisplatin in cancer therapy: molecular mechanisms of action. European journal of pharmacology, 2014. 740: p. 364-378.

59. Horita, N., et al., The best platinum regimens for chemo-naive incurable non-small cell lung cancer: network meta-analysis. Scientific Reports, 2017. 7(1): p. 13185.

60. Ramalingam, S. and C.P. Belani, Taxanes for advanced non-small cell lung cancer. Expert Opinion on Pharmacotherapy, 2002. 3(12): p. 1693-1709.

61. Weaver, B.A., How Taxol/paclitaxel kills cancer cells. Mol Biol Cell, 2014. 25(18): p. 2677-81.

62. Gupta, R.C., et al., Controlled-release systemic delivery - a new concept in cancer chemoprevention. Carcinogenesis, 2012. 33(8): p. 1608-15.

63. Aqil, F., et al., Multi-layer polymeric implants for sustained release of chemopreventives. Cancer Lett, 2012. 326(1): p. 33-40.

64. Cai, Y., et al., Effect of Withaferin A on A549 cellular proliferation and apoptosis in non-small cell lung cancer. Asian Pac J Cancer Prev, 2014. 15(4): p. 1711-4.

65. Gupta, R., et al., Controlled-release systemic delivery - a new concept in cancer chemoprevention. Vol. 33. 2012. 1608-15.

66. Munagala, R., et al., Withaferin A induces p53-dependent apoptosis by repression of HPV oncogenes and upregulation of tumor suppressor proteins in human cervical cancer cells. Carcinogenesis, 2011. 32(11): p. 1697-705.

67. $\mathrm{Yu}, \mathrm{Y}$., et al., Withaferin A targets heat shock protein 90 in pancreatic cancer cells. Biochemical pharmacology, 2010. 79(4): p. 542-551.

68. McKenna, M.K., et al., Anti-cancer activity of withaferin A in B-cell lymphoma. Cancer Biol Ther, 2015. 16(7): p. 1088-98.

69. Patel, N., et al., Rescue of paclitaxel sensitivity by repression of Prohibitinl in drugresistant cancer cells. Proceedings of the National Academy of Sciences, 2010. 107(6): p. 2503.

70. Holleman, A., et al., miR-135a contributes to paclitaxel resistance in tumor cells both in vitro and in vivo. Oncogene, 2011. 30(43): p. 4386-98.

71. Chou, T.-C., Drug Combination Studies and Their Synergy Quantification Using the Chou-Talalay Method. Cancer Research, 2010. 70(2): p. 440. 
72. Dalvi, M.P., et al., Taxane-Platin-Resistant Lung Cancers Co-develop Hypersensitivity to JumonjiC Demethylase Inhibitors. Cell Rep, 2017. 19(8): p. 1669-1684.

73. Rotow, J. and T.G. Bivona, Understanding and targeting resistance mechanisms in NSCLC. Nat Rev Cancer, 2017. 17(11): p. 637-658.

74. Sacco, J.J. and M.J. Clague, Dysregulation of the Met pathway in non-small cell lung cancer: implications for drug targeting and resistance. Translational Lung Cancer Research, 2015. 4(3): p. 242-252.

75. Dalvi, M.P., et al., Taxane-Platin-Resistant Lung Cancers Co-develop Hypersensitivity to JumonjiC Demethylase Inhibitors. Cell reports, 2017. 19(8): p. 1669-1684.

76. Li, X., et al., Synergistic antitumor activity of withaferin A combined with oxaliplatin triggers reactive oxygen species-mediated inactivation of the PI3K/AKT pathway in human pancreatic cancer cells. Cancer Lett, 2015. 357(1): p. 219-30.

77. Hahm, E.R. and S.V. Singh, Withaferin A-induced apoptosis in human breast cancer cells is associated with suppression of inhibitor of apoptosis family protein expression. Cancer Lett, 2013. 334(1): p. 101-8.

78. Pasquier, E., et al., Concentration- and schedule-dependent effects of chemotherapy on the angiogenic potential and drug sensitivity of vascular endothelial cells. Angiogenesis, 2013. 16(2): p. 373-86.

79. Liu, T., et al., Sequence-dependent synergistic cytotoxicity of icotinib and pemetrexed in human lung cancer cell lines in vitro and in vivo. Journal of Experimental \& Clinical Cancer Research, 2019. 38(1): p. 148.

80. Nagai, S., et al., Schedule-Dependent Synergistic Effect of Pemetrexed Combined with Gemcitabine against Malignant Pleural Mesothelioma and Non-Small Cell Lung Cancer Cell Lines. Chemotherapy, 2008. 54(3): p. 166-175.

81. Ruchalski, K.L. and K. Brown, Lung Cancer Screening Update. J Thorac Imaging, 2016. 31(4): p. 190-200.

82. Vinay, D.S., et al., Immune evasion in cancer: Mechanistic basis and therapeutic strategies. Seminars in Cancer Biology, 2015. 35: p. S185-S198.

83. Kyakulaga, A.H., et al., Withaferin A inhibits Epithelial to Mesenchymal Transition in Non-Small Cell Lung Cancer Cells. Sci Rep, 2018. 8(1): p. 15737.

84. Robert, J., [Biology of cancer metastasis]. Bull Cancer, 2013. 100(4): p. 333-42.

85. Siegel, R.L., K.D. Miller, and A. Jemal, Cancer Statistics, 2017. CA Cancer J Clin, 2017. 67(1): p. 7-30.

86. Liam, C.-K., et al., Lung cancer staging now and in the future. Respirology, 2015. 20(4): p. 526-534.

87. Daga, A., et al., Current drugs and drug targets in non-small cell lung cancer: limitations and opportunities. Asian Pac J Cancer Prev, 2015. 16(10): p. 4147-56.

88. Liu, Q., et al., Factors involved in cancer metastasis: a better understanding to "seed and soil" hypothesis. Mol Cancer, 2017. 16(1): p. 176.

89. Eser, P.O. and P.A. Janne, TGFbeta pathway inhibition in the treatment of nonsmall cell lung cancer. Pharmacol Ther, 2017.

90. Gonzalez, D.M. and D. Medici, Signaling mechanisms of the epithelialmesenchymal transition. Sci Signal, 2014. 7(344): p. re8. 
91. Kalluri, R. and R.A. Weinberg, The basics of epithelial-mesenchymal transition. The Journal of Clinical Investigation, 2009. 119(6): p. 1420-1428.

92. Valcourt, U., et al., Analysis of Epithelial-Mesenchymal Transition Induced by Transforming Growth Factor beta. Methods Mol Biol, 2016. 1344: p. 147-81.

93. García de Herreros, A., Epithelial to mesenchymal transition in tumor cells as consequence of phenotypic instability. Frontiers in Cell and Developmental Biology, 2014. 2: p. 71.

94. Nishikawa, Y., et al., Withaferin A Induces Cell Death Selectively in AndrogenIndependent Prostate Cancer Cells but Not in Normal Fibroblast Cells. PLoS One, 2015. 10(7): p. e0134137.

95. Choi, B.Y. and B.W. Kim, Withaferin-A Inhibits Colon Cancer Cell Growth by Blocking STAT3 Transcriptional Activity. J Cancer Prev, 2015. 20(3): p. 185-92.

96. Yang, Z., et al., Withania somnifera root extract inhibits mammary cancer metastasis and epithelial to mesenchymal transition. PLoS One, 2013. 8(9): p. e75069.

97. Wu, Y. and B.P. Zhou, $T N F-\alpha / N F-\kappa B /$ Snail pathway in cancer cell migration and invasion. British Journal of Cancer, 2010. 102(4): p. 639-644.

98. Shibue T, W.R., EMT, CSCs, and drug resistance: the mechanistic link and clinical implications. Nat Rev Clin Oncol, 2017. 14(10): p. 611-629.

99. Bowen Du, J.S.S., Targeting Epithelial-Mesenhymal Transition (EMT) to Overcome Drug Resistance in Cancer. Molecules, 2016. 21(17): p. 965.

100. Choi, B.Y. and B.-W. Kim, Withaferin-A Inhibits Colon Cancer Cell Growth by Blocking STAT3 Transcriptional Activity. Journal of Cancer Prevention, 2015. 20(3): p. 185-192.

101. Parag PS, T.V.D., Leah J. Siskind Levi J Beverly, Common cytotoxic chemotherapeutics induce epithelial-mesenchymal transition (EMT) downstream of ER stress. Oncotarget, 2017. 8(14): p. 22625-22639.

102. Agarwalla, P., et al., Glucocorticoid receptor-mediated delivery of nano goldwithaferin conjugates for reversal of epithelial-to-mesenchymal transition and tumor regression. Nanomedicine (Lond), 2016. 11(19): p. 2529-46.

103. Lee, J., et al., Withaferin A Inhibits Experimental Epithelial-Mesenchymal Transition in MCF-10A Cells and Suppresses Vimentin Protein Level in Vivo in Breast Tumors. Molecular carcinogenesis, 2015. 54(6): p. 417-429.

104. Gemmill, R.M., et al., ZEB1-responsive genes in non-small cell lung cancer. Cancer Lett, 2011. 300(1): p. 66-78.

105. Moustakas, A. and C.H. Heldin, Mechanisms of TGFbeta-Induced EpithelialMesenchymal Transition. J Clin Med, 2016. 5(7).

106. Kamitani, S., et al., Simultaneous stimulation with TGF-betal and TNF-alpha induces epithelial mesenchymal transition in bronchial epithelial cells. Int Arch Allergy Immunol, 2011. 155(2): p. 119-28.

107. Bale, S., et al., An Adaptogen: Withaferin A Ameliorates in Vitro and in Vivo Pulmonary Fibrosis by Modulating the Interplay of Fibrotic, Matricelluar Proteins, and Cytokines. Front Pharmacol, 2018. 9: p. 248.

108. Kawata, M., et al., TGF-beta-induced epithelial-mesenchymal transition of A549 lung adenocarcinoma cells is enhanced by pro-inflammatory cytokines derived from RAW 264.7 macrophage cells. J Biochem, 2012. 151(2): p. 205-16. 
109. Kumar, M., et al., $N F-\kappa B$ Regulates Mesenchymal Transition for the Induction of Non-Small Cell Lung Cancer Initiating Cells. PLoS ONE, 2013. 8(7): p. e68597.

110. Kaileh, M., et al., Withaferin a strongly elicits IkappaB kinase beta hyperphosphorylation concomitant with potent inhibition of its kinase activity. $\mathrm{J}$ Biol Chem, 2007. 282(7): p. 4253-64.

111. Singh D1, A.A., Maurya R, Naik S., Withania somnifera inhibits NF-kappaB and AP-1 transcription factors in human peripheral blood and synovial fluid mononuclear cells. Phytother Res, 2007.

112. Undevia, S.D., G. Gomez-Abuin, and M.J. Ratain, Pharmacokinetic variability of anticancer agents. Nature Reviews Cancer, 2005. 5(6): p. 447-458.

113. Fahrenbruch, R., et al., Dose Rounding of Biologic and Cytotoxic Anticancer Agents: A Position Statement of the Hematology/Oncology Pharmacy Association. Journal of Oncology Practice, 2018. 14(3): p. e130-e136.

114. Paci, A., et al., Review of therapeutic drug monitoring of anticancer drugs part 1 \&\#x2013; Cytotoxics. European Journal of Cancer, 2014. 50(12): p. 2010-2019.

115. Samadi, A.K., Chapter Three - Potential Anticancer Properties and Mechanisms of Action of Withanolides, in The Enzymes, S.Z. Bathaie and F. Tamanoi, Editors. 2015, Academic Press. p. 73-94.

116. Choi, M.J., et al., Endoplasmic reticulum stress mediates withaferin A-induced apoptosis in human renal carcinoma cells. Toxicol In Vitro, 2011. 25(3): p. 692-8.

117. Santagata, S., et al., Using the heat-shock response to discover anticancer compounds that target protein homeostasis. ACS Chem Biol, 2012. 7(2): p. 340-9.

118. Heyninck, K., et al., Withaferin A inhibits NF-kappaB activation by targeting cysteine 179 in IKKbeta. Biochem Pharmacol, 2014. 91(4): p. 501-9.

119. Di, L., et al., Mechanistic insights from comparing intrinsic clearance values between human liver microsomes and hepatocytes to guide drug design. Eur J Med Chem, 2012. 57: p. 441-8.

120. Sherwood, L.C., et al., Development of a goat model for evaluation of withaferin A: Cervical implants for the treatment of cervical intraepithelial neoplasia. Experimental and molecular pathology, 2017. 103(3): p. 320-329.

121. Li, P. and M.G. Bartlett, A review of sample preparation methods for quantitation of small-molecule analytes in brain tissue by liquid chromatography tandem mass spectrometry (LC-MS/MS). Analytical Methods, 2014. 6(16): p. 6183-6207.

122. Patel, S.B., N.J. Rao, and L.L. Hingorani, Safety assessment of Withania somnifera extract standardized for Withaferin A: Acute and sub-acute toxicity study. J Ayurveda Integr Med, 2016. 7(1): p. 30-7.

123. Sharada, A.C., F.E. Solomon, and P.U. Devi, Toxicity of Withania Somnifera Root Extract in Rats and Mice. International Journal of Pharmacognosy, 1993. 31(3): p. 205-212.

124. Choudhary, D., S. Bhattacharyya, and S. Bose, Efficacy and Safety of Ashwagandha (Withania somnifera (L.) Dunal) Root Extract in Improving Memory and Cognitive Functions. Journal of Dietary Supplements, 2017. 14(6): p. 599-612.

125. Patil, D., et al., Determination of withaferin A and withanolide A in mice plasma using high-performance liquid chromatography-tandem mass spectrometry: application to pharmacokinetics after oral administration of Withania somnifera aqueous extract. J Pharm Biomed Anal, 2013. 80: p. 203-12. 
126. Agrawal, P., R. Vegda, and K. Laddha, Simultaneous Estimation of Withaferin A and Z-Guggulsterone in Marketed Formulation by RP-HPLC. J Chromatogr Sci, 2015. 53(6): p. 940-4.

127. Preet, R. and R.C. Gupta, Quantification of withaferin-A and withanolide-A in diploid $(n=12)$ and tetraploid cytotypes $(n=24)$ of "Rassbhary", Physalis angulata L. Natural Product Research, 2018: p. 1-4.

128. Khedgikar, V., et al., Withaferin A: a proteasomal inhibitor promotes healing after injury and exerts anabolic effect on osteoporotic bone. Cell Death \&Amp; Disease, 2013. 4: p. e778.

129. Siegel, R.L., K.D. Miller, and A. Jemal, Cancer statistics, 2018. CA Cancer J Clin, 2018. 68(1): p. 7-30.

130. Malik, F., et al., Reactive oxygen species generation and mitochondrial dysfunction in the apoptotic cell death of human myeloid leukemia HL-60 cells by a dietary compound withaferin A with concomitant protection by $\mathrm{N}$-acetyl cysteine. Apoptosis, 2007. 12(11): p. 2115-33.

131. Hahm, E.R., et al., Withaferin A-induced apoptosis in human breast cancer cells is mediated by reactive oxygen species. PLoS One, 2011. 6(8): p. e23354. 


\title{
CURRICULUM VITAE
}

\author{
Al Hassan Kyakulaga \\ Department of Pharmacology \& Toxicology, University of Louisville \\ 580 S. Preston Street, Rm. 319 \\ Louisville, KY. 40202. Tel.502-654 237 \\ alhassan.kyakulaga@louisville.edu / alhassankyakulaga@yahoo.com
}

\section{EDUCATION:}

2014 - 2019: Ph.D. Pharmacology \& Toxicology, University of Louisville, Louisville, KY. 2014 - 2017: M.S Pharmacology \& Toxicology, University of Louisville, Louisville, KY. 2009 - 2012: MSc. Pharmacology, Makerere University, Kampala, Uganda 2005 - 2009: Bachelor's Degree (BBLT), Makerere University, Kampala, Uganda

\section{TRAINING:}

1. Physiologically Based Pharmacokinetic (PBPK) Modeling Workshop, Colorado State University, Fort Collins, CO. Prof. R.S. Yang. August 2018.

2. LCMS/MS Regional Sci. Dev. Seminar, Agilent Technologies. February 2019

3. Fundamentals of Pharmacokinetics (103-OD, Certara University)

4. NCA Data Analysis (105-OD, Certara University)

5. Introduction to Phoenix WinNonLin (100-OD, Certara University) 


\section{ACADEMIC AND PROFESSIONAL APPOINTMENTS:}

\section{4 - Pres: Graduate Fellow}

Department of Pharmacology \& Toxicology

University of Louisville, Louisville, KY, USA

2013 - Pres: Lecturer

Division of Pharmacology, Toxicology \& Therapeutics

Makerere University, Kampala, Uganda

2009 - 2013: Teaching Assistant

Division of Pharmacology, Toxicology \& Therapeutics

Makerere University, Kampala, Uganda

2008 - 2010: Research Assistant

Clinical Trial on Malaria Prevention Beverage (Artavol)

Uganda National Council for Science \& Technology

\section{MEMBERSHIP IN PROFESSIONAL SOCIETIES}

2017 - Pres: American Association for Cancer Research (AACR)

2014 - Pres: American Society of Pharmacology \& Experimental Therapeutics (ASPET)

2014 - Pres: Society of Toxicology (SOT)

2013 - Pres: Uganda Pharmacological Society (UPharS)

2009 - Pres: Makerere University Academic Staff Association (MUASA)

\section{TEACHING EXPERIENCE:}

Department of Pharmacology \& Toxicology, University of Louisville, Louisville, KY, USA:

- BMSC-807-05: Pharmacology of Adrenergic Agonists and Antagonists, Fall 2018

- BMSC-807-05: Drugs used for Arrhythmias, Heart Failure, and Angina, Fall 2018

- BMSC-807-05: Antihypertensive Drugs, Fall 2018

- BMSC-807-05: Pituitary, Thyroid and Parathyroid Drugs, Fall 2018

- BMSC-807-05: Adrenal Corticosteroids, Fall 2018

- BMSC-807-05: Pharmacology of the Adrenergic Agonists and Antagonists, Fall 2017

- BIOL - 395-01: Basic Pharmacology, Spring 2016

- DHED-402-01: Respiratory System Pharmacology, Fall 2015 
Div. Pharmacology, Toxicology \& Therapeutics, Makerere University, Kampala, Uganda:

- BLT 2101: Introduction to Pharmacokinetics and Pharmacodynamics, 2009-2014

- BLT 2102: General Systems Pharmacology \& Therapeutics, 2009-2014

- BVM2104: General Pharmacology (PK/PD, Cardiovascular, GIT), 2009-2014

- BLT 2204: Introduction to Principles of Toxicology, 2009-2014

- BVM1103: General and Environmental Toxicology, 2009-2014

- BLT 1202: Biomedical Instrumentation Principles, 2009-2014

- BLT 1105: Principles of Quality Assurance \& Control in the Biomedical Laboratory

\section{ACADEMIC AWARDS AND PROFESSIONAL HONORS}

2016: Winner ( $1^{\text {st }}$ place), ResearchLouisville! (Biomedical Sciences Master's Students).

2013: Principal Candidate, Fulbright Science \& Toxicology Award, 2012/2013

2012 Winner ( $2^{\text {nd }}$ Place). Trainee Oral Presentations Award ( $2^{\text {nd }}$ place $)$, Natural Bioactives

Conference, London, Ontario, Canada (07/2012).

2011: Winner, Next Generation of African Academics, (\$20,000 Research Grant), Carnegie

Cooperation of New York, 08/2010-05/2012

2007: Undergraduate Pharmacology Research Funding. Natural Chemotherapeutics Research Laboratory, Ministry of Health

2005: The Government of Uganda Merit-Based Undergraduate Scholarship, 08/2005 08/2009.

2004: Outstanding Science Student, 2004, (Physics, Chemistry, Biology \& Mathematics) M.M. College Wairaka, Uganda.

\section{SELECTED PEER-REVIEWED ARTICLES:}

1. Kyakulaga, A.H., Radha, M, Aqil, F., and Gupta, R (2018). Withaferin A Inhibits Epithelial to Mesenchymal Transition in Non-small Cell Lung Cancer Cells. Scientific Reports, 8 (1).

2. Aqil, F., Radha, M., Jeyabalan, J,. Agrawal, A.K., Lynn, P., Kyakulaga, A.H., and Gupta, C.R (2017). Exosomal delivery of berry anthocyanidins for the management of ovarian cancer

3. Radha, M., Aqil, F., Jeyabalan, J,. Agrawal, A.K., Lynn, P., Mudd, A.M., Kyakulaga, A.H., Manicka, V.V., Inder S.P., and Gupta, C.R (2017). Exosomal formulation of anthocyanidins against multiple cancer types. Cancer Letters. 393

4. Aqil, F., Kauser, H., Agrawal, A.K., Jeyaprakash, J., Kyakulaga, A.H., Munagala, R., and Gupta, R (2016). Exosomal formulation enhances the therapeutic response 
of celasterol against lung cancer. Experimental and Molecular Pathology. 101:1221.

5. Ogwang, P.E., Omujal, F., Agwaya, S.M., Kyakulaga A.H., and Obua, C (2015). Variations in antimalarial components of Artemesia annua Linn from three regions of Uganda. African Health Sciences. 15 (3): 828-834

6. Kyakulaga. A.H, C. Obua., Ogwang P.E., G Nakabonge and E.N Mwavu: Immunomodulatory effect of aqueous extracts of Auricularia sp and Pleurotus sp mushrooms on cyclophosphamide-induced immunosuppression in Wistar rats. British Journal of Pharmaceutical Research 3(4).

7. Kyakulaga A.H., Olia Deo and Ogwang P.E (2011). The wound healing potential of extracts of Ocimum suave and Bidens pilosa in Wistar rats. African Journal of Pharmacy and Pharmacology 5(2): 132-136

8. Ogwang P.E, Nyafuono J., Omujal F., Tumusiime R.H and Kyakulaga A.H (2011). Pre-clinical efficacy and safety of a herbal formulation for the management of wounds. African Health Sciences, 11(3): 524-529.

9. Kyakulaga, A.H., Alinda, T.B., Vudriko, P. and Ogwang, P.E., (2011). In vivo antidiarrheal activity of the ethanolic extracts of Catharanthus roseus Linn in Wistar rats. African Journal of Pharmacy and Pharmacology. 5 (15): 1797-1800.

10. Kyakulaga AH, Ogwang PE, Nannyonga S, Nyafuono J, Tumusiime R. 2012. Antipyretic and analgesics activities of ethanolic extract of Persea americana mill seeds in Wistar albino rats. AJABS 7(1)

\section{PAPERS IN PREPARATION:}

1. Kyakulaga, A.H., Aqil, F., Radha Munagala., and Gupta, R.C (2019). Synergistic combinations of paclitaxel and withaferin A against non-small cell lung cancer cells. (Submitted to Oncotarget; August, 2019)

2. Kyakulaga, A.H., Aqil, F., Bonham C., and Gupta, R.C (2019). Physiologically based pharmacokinetics of Withaferin $A$ and its prediction of human pharmacokinetics. (Under preparation for submission to European $\mathrm{J}$ Pharmaceutical Sciences)

\section{ABSTRACTS AND PRESENTATIONS:}

1. Withaferin A Inhibits EMT in Non-Small Cell Lung Cancer Cells via regulation of Smad2/3 and NFkB Signaling. AACR 2018 Annual Meeting 2018, McCormick Place North/South Chicago, IL. April 14-18, 2018.

2. Withaferin A alone, and in combination with paclitaxel inhibits invasion, migration and metastasis of NSCLC cells. Research Louisville, 2017! October 11, 2017. 
3. Developing risk values and guidance for ethylene glycol monopropyl ether, a high production volume chemical. March 12-16, 2017: SOT 56th Annual Meeting and ToxExpo 2017.

4. Developing risk values and guidance for ethylene glycol monopropyl ether, a high production volume chemical. OVSOT Annual Meeting. October, 2016

5. Withaferin A alone, and in combination with paclitaxel synergistically inhibits the proliferation of NSCLC cells. Research Louisville2016!

6. Immunomodulatory potential of aqueous extracts of Auricularia and Pleurotus Sp. Mushrooms against cyclophosphamide-induced immunosuppression. July 2012. Natural Bioactive Conference, London, Ontario, Canada.

\section{TECHNIQUES, SOFTWARE, AND INSTRUMENTATION}

Non-Compartmental Analysis (NCA) and PK/PD analysis using Phoenix WinNonLin 8.1, oral and intravenous PBPK modeling using Berkeley Madonna and Gastroplus, data analysis and presentation using GraphPad Prism, Calcusyn, SPSS, and Microsoft Excel. Proficiency with Microsoft PowerPoint, Word, and Outlook, as well as Adobe Acrobat (PDF). Analytical and preparative HPLC/UPLC (Shimadzu, Agilent), LCMS/MS (basic knowledge), Flow cytometry, Confocal microscopy, mammalian cell culture, western blotting, qPCR, cell survival assays, migration and invasion assays, mouse and rat models for preclinical efficacy, pharmacokinetics and toxicity studies, in vitro plasma protein binding, and microsomal metabolic stability studies.

\section{SUPERVISION/MENTORSHIP OF UNDERGRADUATE STUDENTS' RESEARCH}

1. Mbabazi Angella (2008). The acute toxicity effects of ethanolic extracts of Bidens pilosa and Ocimu suave in mice. Makerere University.

2. Kibui Pauline (2009). The analgesic and antiinflamatory activity of methanolic extracst of Solanum incanum in Rats. Makerere University.

3. Miyingo Micheal (2009): The Laxative activity of Cassia dydbomotrya in Rats. Makerere University.

4. Biribawa Menya Victoria (2010): The in vivo antihelmintic activity of ethanolic extracts of Bidens pilosa and A. indica. Makerere University

5. Mbuvi Christine (2011). Antibacterial activity of aqueous extracts of Psidium guajava on multi-drug resistant strains of bacteria. Makerere University

6. Mukiibi Karren (2011): The antidiabetic activity of Spathodea campanulata in Wistar albino rats. Makerere university.

7. Nanyonga Shamim (2012). Antipyretic and analgesic activity of Persea americana in mice. Makerere University. 
8. Kawalya Hakim, (2012): The immunomodulatory effect of B.pilosa ethanolic extract in cyclophosphamide-induced immunosuppression in Wistar rats. Makerere University.

9. Dhikusooka Ronald (2013): Hepatoprotective activity of Solanum lycopersicum (tomato) in nevirapine induced hepatotoxicity in Wistar rats. Makerere University. 